\title{
Roadmap
}

\section{Roadmap on structured light}

\author{
Halina Rubinsztein-Dunlop ${ }^{1,21,22}$, Andrew Forbes ${ }^{2,21,22}$, M V Berry ${ }^{3}$, \\ M R Dennis ${ }^{3}$, David L Andrews ${ }^{4}$, Masud Mansuripur ${ }^{5}$, Cornelia Denz ${ }^{6}$, \\ Christina Alpmann ${ }^{6}$, Peter Banzer ${ }^{7}$, Thomas Bauer ${ }^{7}$, Ebrahim Karimi ${ }^{8}$, \\ Lorenzo Marrucci ${ }^{9}$, Miles Padgett ${ }^{10}$, Monika Ritsch-Marte ${ }^{11}$, \\ Natalia M Litchinitser ${ }^{12}$, Nicholas P Bigelow ${ }^{13}$, C Rosales-Guzmán ${ }^{2}$, \\ A Belmonte ${ }^{14}$, J P Torres ${ }^{14,15}$, Tyler W Neely ${ }^{1}$, Mark Baker ${ }^{1}$, \\ Reuven Gordon ${ }^{16}$, Alexander B Stilgoe ${ }^{1}$, Jacquiline Romero ${ }^{17}$, \\ Andrew G White ${ }^{17}$, Robert Fickler ${ }^{8}$, Alan E Willner ${ }^{18}$, Guodong Xie ${ }^{18}$, \\ Benjamin McMorran ${ }^{19}$ and Andrew M Weiner ${ }^{20}$
}

\footnotetext{
${ }^{1}$ School of Mathematics and Physics, The University of Queensland, St Lucia, QLD, 4072, Australia

${ }^{2}$ School of Physics, University of the Witwatersrand, Private Bag 3, Johannesburg 2050, South Africa

${ }^{3}$ H H Wills Physics Laboratory, Tyndall Avenue, Bristol BS8 1TL, UK

${ }^{4}$ School of Chemistry, University of East Anglia, Norwich Research Park, Norwich NR4 7TJ, UK

${ }^{5}$ College of Optical Sciences, The University of Arizona, Tucson, AZ 85721, USA

${ }^{6}$ Institute of Applied Physics, University of Muenster, Corrensstr. 2/4, 48149 Muenster, Germany

${ }^{7}$ Max Planck Institute for the Science of Light, Guenther-Scharowsky-Str. 1/Bldg 24, 91058 Erlangen,

Germany

${ }^{8}$ Department of Physics and Max Planck Centre for Extreme and Quantum Photonics, University of Ottawa, Ottawa, Ontario K1N 6N5, Canada

${ }^{9}$ Dipartimento di Fisica, Università di Napoli Federico II, Complesso Universitario di Monte S. Angelo, via Cintia, 80126 Napoli, Italy

${ }^{10}$ SUPA, School of Physics and Astronomy, University of Glasgow, Glasgow, G12 8QQ, UK

${ }^{11}$ Division of Biomedical Physics, Medical University of Innsbruck, 6020 Innsbruck, Austria

${ }^{12}$ Department of Electrical Engineering, State University of New York, Buffalo, NY 14260, USA

${ }^{13}$ The Institute of Optics, University of Rochester, Rochester, New York 14627, USA

${ }^{14}$ Technical University of Catalonia, Department of Signal Theory and Communications, 08034 Barcelona, Spain

${ }^{15}$ ICFO-Institut de Ciencies Fotoniques, Mediterranean Technology Park, 08860 Castelldefels (Barcelona), Spain

${ }^{16}$ Department of Electrical and Computer Engineering, University of Victoria, Victoria, British Columbia V8P 5C2, Canada

${ }^{17}$ Centre for Quantum Computation and Communication Technology, School of Mathematics and Physics, University of Queensland, Brisbane, Queensland 4072, Australia

${ }^{18}$ Department of Electrical Engineering, University of Southern California, Los Angeles, CA 90089, USA

${ }^{19}$ Department of Physics, University of Oregon, 1585 E 13th Avenue, Eugene, Oregon 97403, USA

${ }^{20}$ School of Electrical and Computer Engineering, Purdue University, West Lafayette, Indiana 47906, USA
}

E-mail: halina@physics.uq.edu.au and Andrew.Forbes@wits.ac.za

Received 11 May 2016, revised 9 September 2016

Accepted for publication 9 September 2016

Published 25 November 2016

\footnotetext{
Abstract

21 Guest editors of the roadmap.

22 Authors to whom any correspondence should be addressed.
}

Structured light refers to the generation and application of custom light fields. As the tools and technology to create and detect structured light have evolved, steadily the applications have begun to emerge. This roadmap touches on the key fields within structured light from the 
perspective of experts in those areas, providing insight into the current state and the challenges their respective fields face. Collectively the roadmap outlines the venerable nature of structured light research and the exciting prospects for the future that are yet to be realized.

Keywords: structured light, tailored light, shaped light, sculpted light, structured illumination, orbital angular momentum, vortices, structured polarisation

(Some figures may appear in colour only in the online journal)

\section{Contents}

1. Introduction 3

2. Vortices, natural and deliberate 4

3. Structured light photonics and light-matter interactions 6

4. Electromagnetic force and momentum 8

5. Electromagnetic angular momentum 10

6. Shaping light 12

7. Structured polarization and its measurement at the nanoscale 14

8. Space-varying polarized fields: classical and quantum prospects 16

9. Imaging with structured light 18

10. Structured light for microscopy 20

11. Structured light on the microscale 22

12. Application of structured light to ultracold atoms: controlling the motion and quantum state, and creating singular optic analogues

13. Structured light for metrology 26

14. Structured light for atomtronic devices 28

15. Nanoaperture tweezers: structured light at the nanoscale 30

16. Structured light optical tweezers 32

17. Quantum information processing with structured light 34

18. Quantum communication using structured light 36

19. Classical optical communication systems using structured light 38

20. Structured electron matter waves 40

21. Structuring light in time 42

22. Tailoring light at the source 44 


\section{Introduction}

\section{Halina Rubinsztein-Dunlop ${ }^{1}$ and Andrew Forbes ${ }^{2}$ \\ ${ }^{1}$ The University of Queensland \\ ${ }^{2}$ University of the Witwatersrand}

Structured light, tailored light, shaped light, sculpted light: all terms that one finds in the literature referring to the generation and application of custom light fields. Invariably the topic is coherent light although it need not be, and the subject matter is the study and application of light with structured intensity, polarization and phase. But these paradigms are shifting as the field matures. In previous decades structured light would refer to intensity patterns at a particular plane (as seen on a camera), created perhaps by diffractive optics; today the tools exist to create custom fields in multiple planes using dynamic and geometric phase control, as recently shown with the 3D polarization Mobius strip. The tools to create have been mirrored by the tools to detect, and steadily applications have begun to emerge. One needs to look no further than the 2014 Nobel prize in Chemistry to see the impact of structured light and its cross-disciplinary influence. A key technology that has enabled this has been spatial light modulators (SLMs), originally designed for projection systems but for a decade now used routinely for generating structured light fields, including more conventional fields such as the eigenmodes of free space, waveguides, fibre optics, and so on. A slightly newer development is the use of digital micromirror devices (DMDs), also originally developed for projection systems that provide the means to produce high speed dynamical structured light fields. It is inevitable that we will see further technological advances to fuel progress, sometimes in a circuitous fashion: SLMs have been exploited in projection, but only to enable single-pixel imaging through structured illumination.

In recent times structured light has become synonymous with orbital angular momentum (OAM). The richness of the topic as well as its myriad of applications has seen it attract much attention. Today the fundamentals of angular momentum remain the subject of debate, 11 while the applications have extended from imaging and $3 \mathrm{D}$ micromanipulation to classical and quantum communication.

Going forward one wonders what exciting prospects await the expansion of structured light concepts beyond the spatial domain, for example, the shaping of light's time envelop and frequency control. Could we see optical fields structured in all dimensions and in all degrees of freedom? The link between imaging and structured light has only recently started to appear, and surely we will see many advances in this field in the future. Intriguingly, the concepts within structured light can be extended and applied to waves in general, and recently there has been active interest in structured electron waves. Might we be able to control matter waves with the same degree of ease as optical fields? With the benefit of shorter wavelengths, could this enable a technology leap in microscopy?

This roadmap also points to the future integration and compactness of solutions. Structured light from the source, or at the micro- and nanoscales, lag behind the tabletop solutions we have today. One might expect a move to miniaturization as application fields mature. And it is applications where we might see the most developments in the future. Most applications today are laboratory demonstrations; might we see technology adoption and commercialization in the future?

This roadmap outlines the venerable nature of the field, touching on the key fields within structured light, but also the exciting prospects for the future that are yet to be realized. 


\section{Vortices, natural and deliberate}

\section{$M V$ Berry and M R Dennis}

University of Bristol

\subsection{Singular optics context}

In coherent light, represented as a complex scalar field in free space, an optical vortex is a place where the phase is undefined: optical vortices are phase singularities. Since the complex amplitude is a single-valued function of position, this can only happen where the intensity is zero (nodes). They are called vortices because the gradient of phase (local wavevector, i.e. optical momentum) circulates around them $[1,2]$.

Optical vortices occur typically ('generically') in the absence of any special conditions; they are natural structures [3]. They also occur in light that is deliberately structured, for example, in cylindrical light beams with a uniformly increasing phase around the axis (such as Laguerre-Gauss beams). Around the axis of such beams, the phase changes by $\pm 2 \pi \ell$ for integer $\ell$ ( $\ell$ defines the vortex strength). Natural vortices, such as those in figure 1 , only have strength \pm 1 . Under perturbation a higher-order vortex of strength $\ell$ breaks into a cluster of $\ell$ typical ones.

In a $2 \mathrm{D}$ plane, such as an observation screen, typical vortices are points. In 3D space, they are lines, threads of darkness, a ghostly skeleton of typically complicated wavefields. On optical vortices, wavefronts (constant-phase surfaces) end, leading to an analogy with crystal dislocations, where planes of atoms end; optical vortices are wave dislocations [1].

Optical vortices occur in complex scalar fields representing propagating waves. They should be distinguished from the nodal lines (2D) and surfaces (in 3D) in the realvalued fields representing standing waves, such as cavity modes. The 'dark interference fringes' in diffraction patterns, considered in conventional optics, are also surfaces in 3D, but are approximations; perfect destructive interference typically occurs only on lines, which of course are the optical vortices.

The study of optical vortices is part of the larger subject of singular optics [4]. On the simpler level of geometrical optics, in which light is represented by fields of rays (which neglect the phase of light waves), the singularities are different: they are caustics, i.e. bright surfaces in space on which the light is focused. Their typical structures are classified by catastrophe theory. Caustics and optical vortices are complementary singularities. Caustics are prominent high-intensity features, in contrast to vortices where complete destructive interference occurs, nestled in low-intensity regions. On the deeper level, where light is represented by electromagnetic vector fields, the singularities are different again, for example, lines on which the polarization is purely circular or purely linear (and typically different for the electric and magnetic fields) [3].

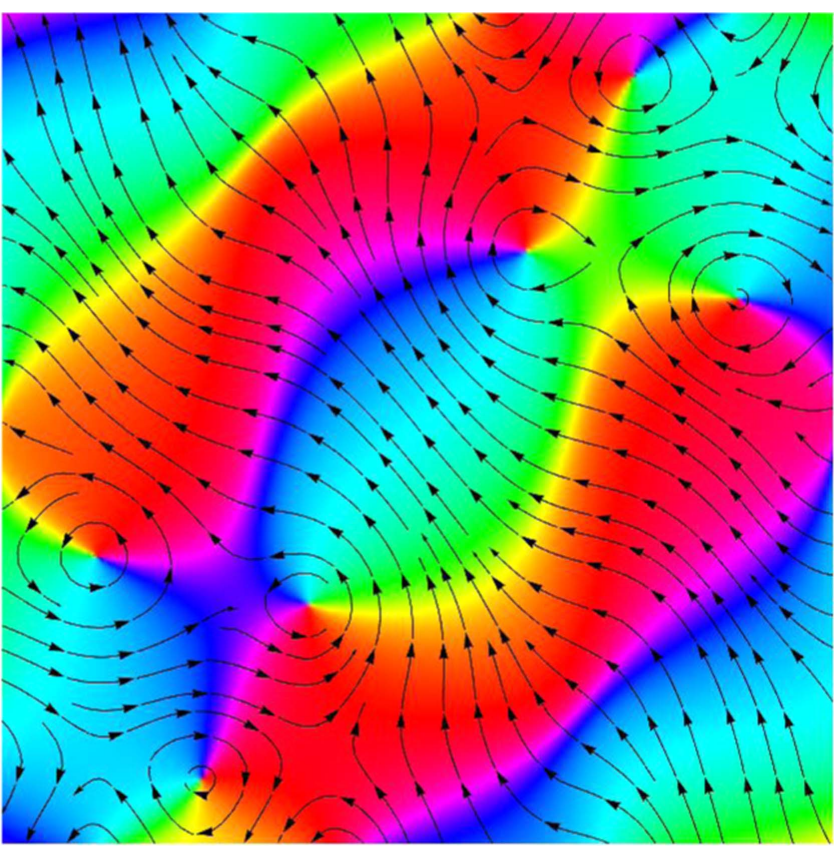

Figure 1. Six optical vortices in two square wavelengths of a natural wavefield: a superposition of ten plane waves with random strengths and phases, with phase colour-coded by hue and streamlines (local wavevector directions) indicated by arrows.

\subsection{Looking back}

Vortices as phase singularities occur throughout wave physics. They seem to have been first identified by Whewell in 1833 in the tides, i.e. moon-driven ocean waves circling the globe, as ('amphidromic') points of no tide, from which the cotidal lines (the wavefronts, loci of places where the tide is instantaneously high) emanate like spokes of a wheel $[1,3]$. In quantum mechanics they were identified by Dirac as emanating from conjectured magnetic monopoles as quantized vortices in superfluids, quantized flux in superconductors (in many-body wavefunctions satisfying equations that need not be linear), in atomic and molecular wavefunctions [5] and in electron beams [6] created by methods analogous to optical vortex beams.

In optics, they were identified in light near dielectric boundaries, near focal points of lenses with aberrations, in edge diffraction, and in superpositions of plane waves. More generally, as ubiquitous features of all types of waves, they were identified in 1974 in a study stimulated by ultrasound scattered from crinkly metal foil (a model of radio waves reflected by the rough terrain beneath the ice covering Antarctica) [1]. In optics, vortices began to be explored experimentally in the 1990s in structured light created using fork holograms, spiral phase plates, cylindrical lenses or SLMs [2, 7].

Also, in the 1990s extensive studies began of the OAM of light, associated with the spatial structure of optical fields [2]. Optical vortices and OAM are distinct but have overlapping concepts. In deliberately structured light, eigenstates of OAM, with rotationally symmetric intensity and azimuthal phase increasing uniformly, are centrally threaded by optical 
vortices. However, in typical light beams, paraxial or not, the total OAM per unit length need not be related to the number of vortices threading the beam.

\subsection{Looking ahead}

In optics, as elsewhere, the morphology of vortex lines in three dimensions pose difficult problems. In random speckle patterns in $3 \mathrm{D}$, it seems that most vortices are closed, and some progress has been made in understanding the distribution of loop lengths. More interesting, and more challenging, are topological statistics involving the probabilities of knotting and linking in naturally occurring waves. Special superpositions of simpler modes, such as Laguerre-Gauss or Bessel beams, do contain knotted and linked vortex loops (figure 2) [8].

A promising application is the vortex coronagraph, in which an optical element consisting of an even-order phase vortex expels the light from a parent star imaged on-axis, allowing observation of its much fainter planet [9]. Underlying this technique is surprising new mathematics.

An application that already exists but which will undoubtedly be developed further occurs in stimulated emission depletion (STED) super-resolution microscopy [10]. Almost all the conventionally diffraction-limited light emitted by a specimen is suppressed by a second illuminating beam, which contains a nodal point (optical vortex) permitting observation of small regions of the image. The technique works because although classical optics restricts the smallness of bright (e.g. focal) regions, dark regions can be arbitrarily small.

The reduced scattering of photons in dark regions makes the existence of singular points or lines with exact intensity nulls (i.e. optical vortices) a desirable property in the optical trapping of ultracold atoms and Bose-Einstein condensates [11].

There are several ways in which the theory of optical vortices, and structured light more generally, intersect the theory and formalism of quantum weak measurement. The longitudinal and transverse shifts in the position and direction of light reflected from dielectric interfaces has been interpreted in this way, leading to predictions of new effects, not yet observed, in beams containing vortices as well as those that do not.

The large local wavevectors (phase gradients) near optical vortices correspond quantum-mechanically to large momenta. These are predicted to transfer large, though rare, 'superkicks' to atoms in the field. This quantum deconstruction of classical radiation pressure (the patter of photons on average) into large rare events near vortices has also not yet been observed.

Another quantum effect, on excited atoms near vortices, is that their darkness could be weakly illuminated by spontaneous emission into unpopulated vacuum states. The size of

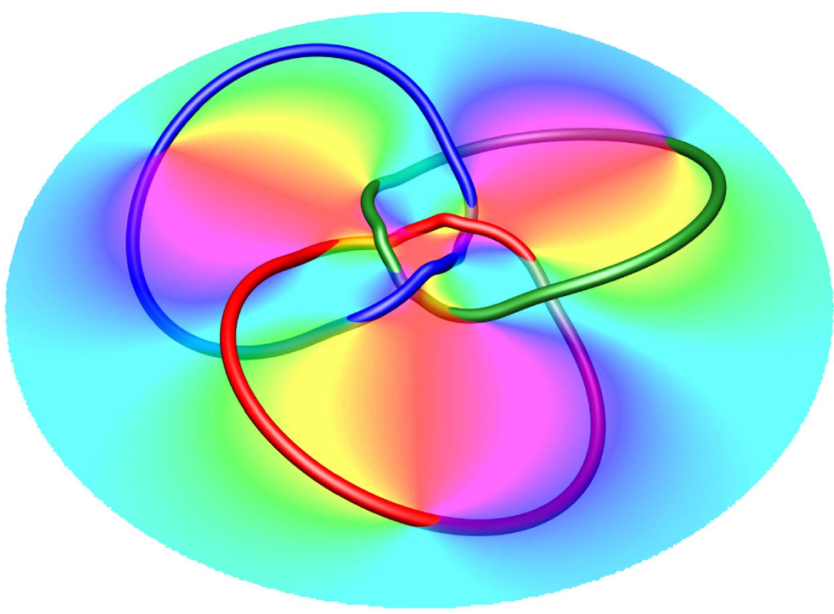

Figure 2. Three optical vortex loops forming Borromean rings, unlinked pairwise but linked triply. (Courtesy of Robert King.)

the corresponding quantum core has been calculated, but the effect has not yet been observed. The analogous effect near the polarization singularities of vector waves has not been investigated.

\subsection{Concluding remarks}

As natural features of wave interference, optical vortices are intrinsic to all sufficiently complex structured light, and their existence and properties in different optical phenomena underpin the physical understanding of the spatial interference patterns.

The complementarity between vortices as phase singularities and caustics as ray singularities reflects the asymmetry of darkness and light, and both are intertwined in naturally and deliberately structured light. As discussed, there are many existing and potential applications for optical vortices, both as intensity nodes and phase singularities.

Being objects of purely classical, monochromatic light, there is no direct connection between optical vortices and properties of photons. Quantum states of light, prepared in simple vortex-carrying modes, such as Laguerre-Gaussian beams, carry quantized OAM in units of $\hbar$ and the quantum vacuum may shine through in the 'quantum cores' of optical vortices [2].

Analogy is a powerful principle in physics, and optical vortices, as the counterparts of vortices in hydrodynamics and matter waves, may yet be the focus of deeper connections between these areas.

\section{Acknowledgments}

Our research is supported by the Leverhulme Trust. 


\section{Structured light photonics and light-matter interactions}

\author{
David L Andrews \\ University of East Anglia
}

\section{Status}

Every interaction between light and matter has the capacity, in principle, to reveal fundamental properties of one or other component. In the early course of developing light beams with intrinsic modal structure (as distinct from projected illumination) there were some expectations that suitably structured light might lead to new fundamental kinds of coupling with matter, revealing different mechanisms, and signalling new opportunities for molecular spectroscopy and sensing. Although a number of those expectations have fallen by the wayside-mostly on the grounds that such effects have proved too subtle and of too small a magnitude to be experimentally worthwhile - the exploration of a variety of other interactions with matter has proved remarkably fruitful.

In the case of optical vortices, for example (see section 2), one suggestion given early prominence was the potential use of light with a twisted wavefront-as illustrated in figure 3-to differentially respond to the sense of handedness associated with chiral materials. Although this specific idea has failed to produce viable applications, current progress on chiroptical phenomena has undoubtedly been enriched by a renewed focus of attention [12]. At the turn of this century, it might have been considered that the optical mechanisms for probing molecular chirality were thoroughly well understood and already at the limit of practical application. Such certainties have been thwarted by a range of clever studies revealing the occurrence of pronounced circular polarization effects at surfaces, metallic nanostructures or near mirrors [13]. Vector polarization modes are also opening up new territory, through their capacity to controllably deliver position-dependent, variable polarization [14].

To date, the most important applications of structured light as a practical tool have arisen in other, quite different areas. Most attention has been divided between the quantum informatics aspects of optical communication, and mechanical effects such as microparticle trapping, tweezing, ordering and wrenching (see especially sections 15 and 16); the latter including rheological applications for viscosity measurement [15]. More exotic structured beam effects on matter-especially those connected with vortex features-have also been identified in a variety of electronic and plasmonic interactions.

The informatics aspects of structured light hold some of the greatest promise (see sections 17-19). For light of a given wavelength, travelling in a given direction, the prospect of each photon conveying additional information, beyond that afforded by the simple binary basis of polarization, appears highly attractive [16]. Such considerations are not restricted to cryptographic security; in the wider pursuit of high information density per channel, additional capacity can prove highly

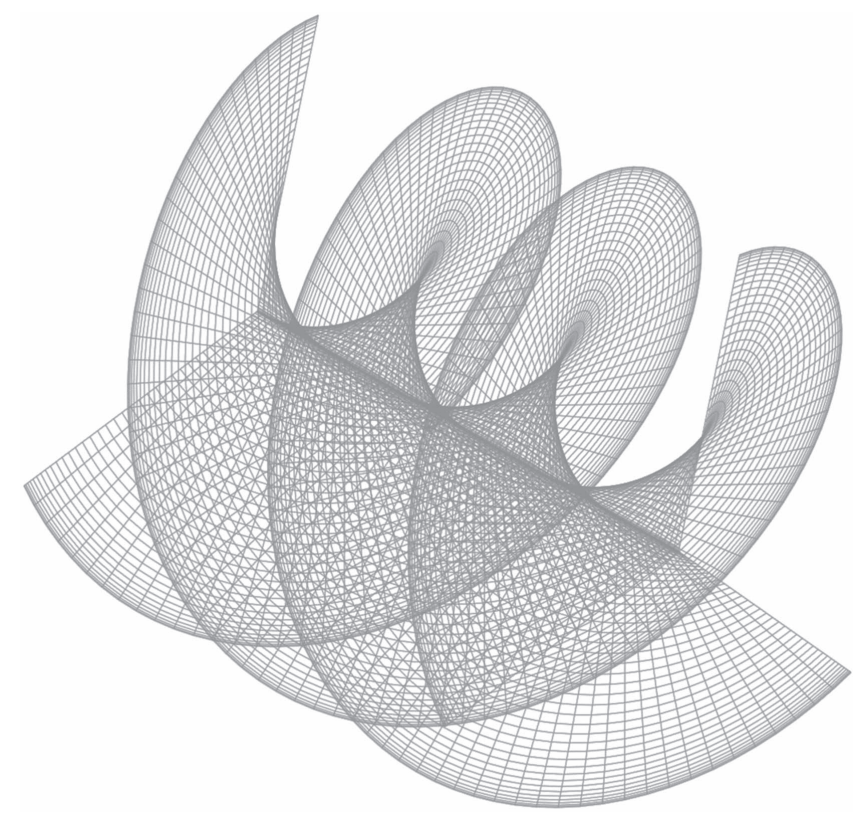

Figure 3. Wavefront surface over the span of three wavelengths for an optical vortex with a topological charge of 3 (three intertwined helical surfaces).

advantageous in reducing the photon budget and hence the overall cost of data transmission.

\section{Current and future challenges}

While experimental research and technical progress continue apace, there are considerable challenges to be addressed in the realm of theory. It is arguable that some developments in structured light are sufficiently extensive to warrant a rewrite of many textbook optics. In this respect there are two tiers of issue. At the most fundamental level there are new, unresolved questions about the character of the photon, and the capacity of widely accepted photon representations to adequately capture the properties of structured beams as conveyed by classical representations [17]. At the very least, these issues invite a thorough reappraisal of assumptions underlying conventional theory (and also those that underpin the type of calculations performed by some computational electrodynamics software). But above that level, there is a wider panoply of features concerning specific aspects of structured light interactions with matter. Very few of the emerging applications are as of yet represented in significant works of reference.

Robert Boyd, an eminent figure in modern optics, has commented that structured light should serve as a near-perfect test case for the study of the quantum-classical boundary, given that structured electromagnetic fields may possess a higher information content per photon than is afforded by a conventional Gaussian beam. Essentially two of the usual three spatial degrees of freedom can be redistributed into the assignment of transverse structure, yet it is not yet clear what limits might be imposed upon the encoding of information by such means, nor where such constraints might originate. In what sense, for example, might the properties of a beam with 
a high topological charge (considered as a quantum number) correspond to a classical limit? What potential features might emerge through quantum entanglement between polarization and spatial degrees of freedom, what scope is there to exploit both a wavevector structure and the vectorial polarization of a Poincaré vortex beam? There are also questions to explore in establishing the relationship between the singularities associated with these two types of beam structure (see section 8).

In the interactions of structured light with matter, some of the other pressing research issues have particular relevance to data transmission, for example how to understand and properly model the effects of photon propagation through media that exhibit density fluctuations or turbulence. Even the fundamental mechanisms for photon emission and detection demand fresh scrutiny, especially if coherent sources of structured radiation are to be seriously developed.

\section{Advances in science and technology to meet challenges}

Some of the issues identified above might in principle be resolved by experiment; for others, even the devising of suitable experiment is a challenge. Of course, experimental studies can prove inconclusive, whilst others may lead to unexpected disputes over interpretation, most notably the question of whether genuinely useful enhancements of data transmission rates can be achieved using OAM-endowed radio waves [18].

It generally takes state of the art technology to prove or disprove the existence of a conjectured phenomenon. For example, while the continued development of q-plates and SLMs paves the way for more precise tailoring of optical beam structures, the most welcome development would be a turnkey laser system for directly delivering beams with a programmable structure, ideally one with tailored fibre optic beam delivery. But even to explore the possibility of directly generating structured light, as for example from molecular arrays [19] or specifically tailored plasmonic metasurfaces [20, 21], already places high demands on current nanofabrication facilities. Addressing the intriguing postulate that twisted beams might indirectly induce nuclear hyperpolarization for magnetic resonance applications is another quest that requires an especially clever integration of nuclear magnetic resonance and laser instrumentation [22].

From the perspective of a theoretician, it seems timely and necessary for the photon-based theory of structured light to be further consolidated into a rigorous and broadly agreed framework before further extensions can reliably identify additional areas of application. This whole field owes its development to a handful of original theory papers, and theory will undoubtedly once again pave the way for new developments. In particular, there is a pressing need to ensure that commercial electrodynamics software, given its widespread deployment by experimentalists, is written in terms of code that properly accommodates forefront theory, and correctly accounts for quantum aspects.

\section{Concluding remarks}

Structured light has emerged as a new and vibrant topicnow almost a distinct discipline-within modern optics. Alongside beam-intrinsic features such as phase singularity, OAM, transverse structure, structured polarization, essentially diffraction-free propagation and beam steering, it offers a wide range of novel opportunities to manifest novel features in its interactions with matter. Moreover the field has already led to advances in atom optics and parallels in the fields of electron optics [6], amongst others (see sections 12 and 20). At this stage in development, notwithstanding some already proven applications, there remains a wide range of questions to furnish further research, both in terms of theory and experiment. Current progress heralds a future in which beams of arbitrary structure can be produced and used to more precisely manipulate matter, and to discern new kinds of information through the exploitation of novel optical mechanisms for sample interrogation. 


\section{Electromagnetic force and momentum}

\section{Masud Mansuripur}

The University of Arizona, Tucson

\section{Status}

At the foundation of the classical theory of electrodynamics lies Maxwell's macroscopic equations, namely,

$$
\begin{gathered}
\nabla \cdot \mathbf{D}(\mathbf{r}, t)=\rho_{\text {free }}(\mathbf{r}, t) . \\
\nabla \times \mathbf{H}(\mathbf{r}, t)=\mathbf{J}_{\text {free }}(\mathbf{r}, t)+\partial \mathbf{D}(\mathbf{r}, t) / \partial t \\
\nabla \times \mathbf{E}(\mathbf{r}, t)=-\partial \mathbf{B}(\mathbf{r}, t) / \partial t . \\
\nabla \cdot \boldsymbol{B}(\boldsymbol{r}, t)=0 .
\end{gathered}
$$

In the above equations, $\rho_{\text {free }}$ and $\boldsymbol{J}_{\text {free }}$ are densities of free charge and free current, displacement $\boldsymbol{D}$ is related to the electric field $\boldsymbol{E}$, polarization $\mathbf{P}$ and permittivity $\varepsilon_{0}$ of free space via $\boldsymbol{D}=\varepsilon_{0} \boldsymbol{E}+\boldsymbol{P}$, and magnetic induction $\boldsymbol{B}$ is related to the magnetic field $\boldsymbol{H}$, magnetization $\mathbf{M}$ and permeability $\mu_{0}$ of free space via $\boldsymbol{B}=\mu_{0} \boldsymbol{H}+\boldsymbol{M}$. These equations tie the electromagnetic (EM) fields $\boldsymbol{E}(\boldsymbol{r}, t)$ and $\boldsymbol{H}(\boldsymbol{r}, t)$ to their sources $\rho_{\text {free }}, \boldsymbol{J}_{\text {free }}, \boldsymbol{P}$, and $\boldsymbol{M}$, which generally have arbitrary distributions throughout space-time $(\boldsymbol{r}, t)$.

Maxwell's equations are silent as to the energy content of the EM fields. However, once the Poynting vector $\boldsymbol{S}(\boldsymbol{r}, t)=\boldsymbol{E}(\boldsymbol{r}, t) \times \boldsymbol{H}(\boldsymbol{r}, t)$ is defined as the universal expression of the flow-rate of EM energy, equations (1)-(4) lead straightforwardly to Poynting's theorem, that is,

$$
\begin{aligned}
& \boldsymbol{\nabla} \cdot \boldsymbol{S}+\frac{\partial}{\partial t}\left(1 / 2 \varepsilon_{0} \boldsymbol{E} \cdot \boldsymbol{E}+1 / 2 \mu_{0} \boldsymbol{H} \cdot \boldsymbol{H}\right) \\
& \quad+\left(\boldsymbol{E} \cdot \boldsymbol{J}_{\text {free }}+\boldsymbol{E} \cdot \frac{\partial \boldsymbol{P}}{\partial t}+\boldsymbol{H} \cdot \frac{\partial \boldsymbol{M}}{\partial t}\right)=0 .
\end{aligned}
$$

Not only does the above equation yield expressions for the field energy densities $\left(\frac{1}{2} \epsilon_{0} E^{2}\right.$ and $\left.\frac{1}{2} \mu_{0} H^{2}\right)$ as well as the energy-exchange rate between material media and the $\boldsymbol{E}$ and $\boldsymbol{H}$ fields (that is, $\boldsymbol{E} \cdot\left(\boldsymbol{J}_{\text {free }}+\partial \boldsymbol{P} / \partial t\right)$ and $\left.\mathbf{H} \cdot \partial \mathbf{M} / \partial t\right)$, but also its form (as a continuity equation) guarantees the conservation of EM energy.

Similarly, the EM force and momentum do not emerge naturally from Maxwell's equations. However, once a stress tensor is defined, equations (1)-(4) can be invoked to arrive at expressions for the densities of the EM force, torque, momentum, and angular momentum [23, 24]. As an example, let us consider the Einstein-Laub stress tensor

$$
\overleftrightarrow{\mathcal{T}}_{\mathrm{EL}}(\mathbf{r}, t)=1 / 2\left(\varepsilon_{\mathrm{o}} \boldsymbol{E} \cdot \mathbf{E}+\mu_{\mathrm{o}} \mathbf{H} \cdot \mathbf{H}\right) \overleftrightarrow{\mathbf{I}}-\boldsymbol{D E}-\boldsymbol{B} \boldsymbol{H},
$$

where $\overleftrightarrow{\mathbf{I}}$ is the second rank identity tensor, a $3 \times 3$ matrix [25]. This stress tensor can be readily shown to satisfy the continuity equation [26]

$$
\boldsymbol{\nabla} \cdot \overleftrightarrow{\mathcal{T}}_{\mathrm{EL}}+\frac{\partial}{\partial t}\left(\boldsymbol{E} \times \boldsymbol{H} / c^{2}\right)+\boldsymbol{F}_{\mathrm{EL}}(\boldsymbol{r}, t)=0,
$$

where the Einstein-Laub force-density $\boldsymbol{F}_{\mathrm{EL}}$ is given by

$$
\begin{aligned}
\boldsymbol{F}_{\mathrm{EL}}(\boldsymbol{r}, t)= & \rho_{\text {free }} \boldsymbol{E}+\boldsymbol{J}_{\text {free }} \times \mu_{\mathrm{o}} \boldsymbol{H}+(\boldsymbol{P} \cdot \boldsymbol{\nabla}) \boldsymbol{E} \\
& +\frac{\partial \boldsymbol{P}}{\partial t} \times \mu_{\mathrm{o}} \boldsymbol{H}+(\boldsymbol{M} \cdot \boldsymbol{\nabla}) \boldsymbol{H}-\frac{\partial \boldsymbol{M}}{\partial t} \times \varepsilon_{\mathrm{o}} \boldsymbol{E} .
\end{aligned}
$$

The formulation proposed by Einstein and Laub thus assigns to the EM field the momentum density, $\boldsymbol{p}_{\mathrm{EL}}(\boldsymbol{r}, t)=\boldsymbol{S}(\boldsymbol{r}, t) / c^{2}$, which has been associated with the name of Max Abraham [27]. The Einstein-Laub force-density $\boldsymbol{F}_{\mathrm{EL}}$ is the force exerted by the $\boldsymbol{E}$ and $\boldsymbol{H}$ fields on material media, which are the seats of $\rho_{\text {free }}, \boldsymbol{J}_{\text {free }}, \boldsymbol{P}$, and $\boldsymbol{M}$. Considering that 'force' is the rate of transfer of mechanical momentum to (or from) material media, the continuity equation (7) guarantees the conservation of linear momentum.

If the position-vector $\boldsymbol{r}$ is cross-multiplied into equation (7), we arrive [26] at a similar equation for the conservation of angular momentum, where the EM angular momentum density will be given by $\mathcal{L}_{\mathrm{EL}}(\boldsymbol{r}, t)=$ $\boldsymbol{r} \times \boldsymbol{S}(\boldsymbol{r}, t) / c^{2}$ and the EM torque-density will be

$$
\boldsymbol{T}_{\mathrm{EL}}(\boldsymbol{r}, t)=\boldsymbol{r} \times \boldsymbol{F}_{\mathrm{EL}}+\boldsymbol{P} \times \mathbf{E}+\boldsymbol{M} \times \boldsymbol{H} .
$$

It must be emphasized that the above discussion is completely general, depending in no way on the nature of the sources. The material media hosting the sources could respond linearly or nonlinearly to the EM fields, they could be mobile or stationary, they could have permanent polarization and magnetization, or their $\boldsymbol{P}$ and $\boldsymbol{M}$ could be induced by local or non-local fields, etc. Maxwell's macroscopic equations and the stress tensor formulation of force, torque, and momentum are applicable under all circumstances [28].

There exists several other stress tensors, each with its own expressions for the densities of EM force, torque, momentum, and angular momentum [23, 27]. For instance, in the standard (Lorentz) formulation of classical electrodynamics, the stress tensor is defined as

$$
\begin{aligned}
{\overleftrightarrow{\mathcal{T}_{L}}}_{(\boldsymbol{r}, t)=} & \frac{1}{2}\left(\varepsilon_{\mathrm{o}} \boldsymbol{E} \cdot \boldsymbol{E}+\mu_{\mathrm{o}}^{-1} \boldsymbol{B} \cdot \boldsymbol{B}\right) \overleftrightarrow{\mathbf{I}} \\
& -\varepsilon_{\mathrm{o}} \boldsymbol{E} \boldsymbol{E}-\mu_{\mathrm{o}}^{-1} \boldsymbol{B B},
\end{aligned}
$$

whereas Minkowski's stress tensor is

$$
\overleftrightarrow{\mathcal{T}}_{M}(\boldsymbol{r}, t)=\frac{1}{2}(\boldsymbol{D} \cdot \boldsymbol{E}+\boldsymbol{B} \cdot \boldsymbol{H}) \overleftrightarrow{\mathbf{I}}-\boldsymbol{D E}-\boldsymbol{B H} .
$$

Each formulation, of course, complies with the conservation laws, provided that the relevant entities are properly defined in accordance with continuity equations such as equations (5) and (7). Occasional confusion in the literature can be traced to the fact that some authors use, for instance, the momentum density from one formulation and the forcedensity from another. Needless to say, if the various entities are used properly (i.e. in the context of a single stress tensor), there should be no confusion and no inconsistency. That is not to say that all the existing formulations are equivalent. There are theoretical arguments (e.g., the Balazs thought experiment [29]) which reveal that certain formulations violate well-established physical principles [30]. Other formulations might require the introduction of so-called 'hidden' 


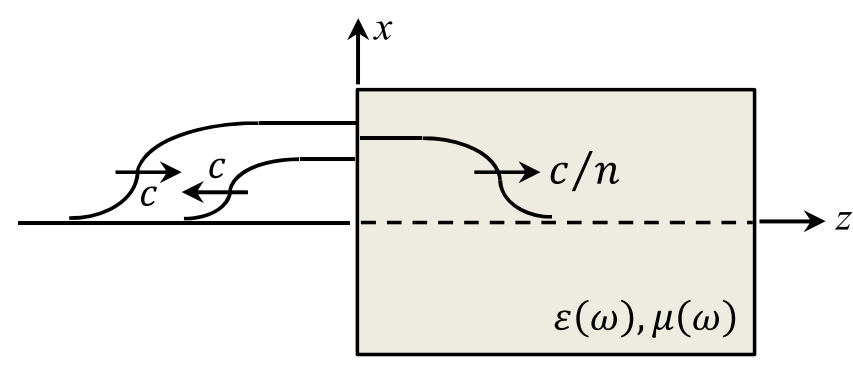

Figure 4. Linearly polarized light pulse of frequency $\omega$ entering a homogeneous, isotropic slab identified by its permittivity $\varepsilon(\omega)$ and permeability $\mu(\omega)$. The incidence medium is free space, in which the incident and reflected pulses propagate at the vacuum speed of light, $c$. Inside the slab, the pulse propagates at the reduced velocity $c / n$, where the refractive index is given by $n=\sqrt{\mu \varepsilon}$.

entities such as hidden energy and hidden momentum $[26,31,32]$. Of course, the ultimate proof of validity of a physical theory is its compliance with experimental observations. The debate as to which stress tensor represents physical reality is very much alive today and the interested reader should consult the vast literature on the subject [23, 24, 27, 32-37]. The present author believes that several appealing features of the Einstein-Laub theory recommend it as a universal theory of EM force and momentum $[26,28,38]$, but there are others who prefer alternative formulations [27, 32, 33].

The following example illustrates the type of results that one can obtain by a consistent application of a specific stress tensor to a given physical problem.

Example: figure 4 shows a light pulse of frequency $\omega$ entering a transparent slab of material identified by its permittivity $\varepsilon(\omega)$ and permeability $\mu(\omega)$. The slab's refractive index and impedance are $n=\sqrt{\mu \varepsilon}$ and $\eta=\sqrt{\mu / \varepsilon}$; the Fresnel reflection coefficient at the entrance facet of the slab is $\rho=(\eta-1) /(\eta+1)$.

The incident beam, whose cross-sectional area is $A$, is linearly polarized along the $x$-axis, its $\boldsymbol{E}$ and $\boldsymbol{H}$ fields being $E_{0} \hat{\boldsymbol{x}} \cos \left(k_{0} z-\omega t\right) \quad$ and $H_{0} \hat{\boldsymbol{y}} \cos \left(k_{0} z-\omega t\right)$, where $H_{0}=E_{0} / Z_{0}$ and $k_{0}=\omega / c$; here $Z_{0}=\sqrt{\mu_{0} / \varepsilon_{0}}$ is the impedance of free space. Considering that the time-rate of arrival of EM momentum at the entrance facet is $1 / 2 \varepsilon_{0} E_{0}^{2} A$, the rate at which EM momentum is delivered to the slab is $\frac{1}{2}\left(1+\rho^{2}\right) \epsilon_{0} E_{0}^{2} A$.

Immediately after the entrance facet, the field amplitudes entering the slab are $E_{1}=(1+\rho) E_{0}$ and $H_{1}=(1-\rho) H_{0}$, yielding the corresponding EM momentum density $\mathscr{P}_{\mathrm{EL}}=\frac{1}{2}\left(1-\rho^{2}\right) \varepsilon_{0} \boldsymbol{E}_{0}^{2} / c$. Given that the velocity of the leading edge of the pulse within the slab is $c / n$, the (Abraham) momentum content of the slab increases at the rate of $\frac{1}{2}\left(1-\rho^{2}\right) \epsilon_{0} E_{0}^{2} A / \sqrt{\mu \epsilon}$. The difference between the rate of delivery of EM momentum from the outside and the growth rate of EM momentum within the slab, namely $(\varepsilon+\mu-2) \varepsilon_{0} E_{0}^{2} /(\sqrt{\varepsilon}+\sqrt{\mu})^{2}$, must be taken up by the EM force exerted on the slab at the leading edge of the light pulse. This is indeed the case, as can be readily verified by integrating the force density $\boldsymbol{F}_{\mathrm{EL}}(\boldsymbol{r}, t)$ of equation (8) over the volume of the slab. Calculation of this force requires defining the function $f(z)$ to represent the fields inside the slab as $\boldsymbol{E}(z, t)=E_{1} \hat{\boldsymbol{x}} f(z-c t / n)$ and $\boldsymbol{H}(z, t)=H_{1} \hat{\boldsymbol{y}} f(z-c t / n)$; material polarization and magnetization now become $\boldsymbol{P}(z, t)=$ $\varepsilon_{0}(\varepsilon-1) \boldsymbol{E}(z, t)$ and $\mathbf{M}(z, t)=\mu_{0}(\mu-1) \mathbf{H}(z, t)$ (note that material dispersion is ignored). Only the fourth and sixth terms on the right-hand side of equation (8) contribute to the force density in the present example. The requisite calculations are fairly elementary and need not be elaborated here.

Finally, if the pulse duration is denoted by $\tau$, the total energy and momentum delivered to the slab, namely

$$
\begin{aligned}
& \mathcal{E}=\frac{1}{2}\left(1-\rho^{2}\right) Z_{0}^{-1} E_{0}^{2} A \tau, \\
& \mathscr{P}=\frac{1}{2}\left(1+\rho^{2}\right) \varepsilon_{0} E_{0}^{2} A \tau \hat{z},
\end{aligned}
$$

yield $\mathscr{P} / \mathcal{E}=\frac{1}{2} \sqrt{\mu_{0} \varepsilon_{0}}(\sqrt{\mu / \varepsilon}+\sqrt{\varepsilon / \mu})$. Thus, for a single photon of energy $\hbar \omega$ travelling in the slab, the total (i.e. electromagnetic + mechanical) momentum is bound to be $(\sqrt{\mu / \varepsilon}+\sqrt{\varepsilon / \mu}) \hbar \omega / 2 c$ [28].

\section{Current and future challenges}

On the fundamental side, there is a need to clarify the differences among the various stress tensor formulations, namely, those of Lorentz, Minkowski, Abraham, EinsteinLaub, and Chu [23, 27, 33]. Contrary to a widely held belief, these tensors can be distinguished from each other by the subtle differences in their predicted distributions of force and/or torque density in deformable media [38]. Of particular interest are experiments on transparent (i.e. nonabsorbing) magnetic materials, as some of the major differences among the proposed stress tensors emerge in magnetized or magnetizable media [26, 27, 37].

\section{Advances in science and technology to meet challenges}

The observable effects of EM force and torque on material media are enhanced when the fields are made to interact with micro- and nanostructured objects, especially those possessing extremely large quality factors, also known as high- $Q$ resonators. Quantum opto-mechanics has blossomed in recent years as a result of advances in micro/nanofabrication. These advances are likely to continue as the fabrication tools and measurement techniques improve, and as novel metamaterials are discovered.

\section{Concluding remarks}

The classical theory of electrodynamics suffers from an embarrassment of riches in that several stress tensors have been proposed over the years that provide alternative expressions for EM force, torque, and momentum in material media [26, 27]. Advances in metamaterials, micro-structured material fabrication, and measurement tools and techniques have reached a stage where it is now possible to expect that experiments in the near future will be able to distinguish among the extant EM stress tensors. 


\section{Electromagnetic angular momentum}

\section{Masud Mansuripur}

The University of Arizona, Tucson

\section{Status}

In addition to energy and linear momentum, EM fields can carry spin angular momentum (when circularly or elliptically polarized), and also orbital angular momentum (OAM) (for example, in the presence of phase vorticity) [39-41]. The classical theory of electrodynamics does not distinguish between the two types of angular momentum, treating them on an equal footing [4246]. In quantum optics, however, while a single photon may possess only one $\hbar$ of spin, it has the capacity to carry $m \hbar$ of OAM, $m$ being an arbitrary integer, or, in special cases, a halfinteger [47]. As usual, $\hbar$ is Planck's reduced constant.

In section 4 on EM momentum, we point out that the specification of a stress tensor uniquely identifies, in conjunction with Maxwell's macroscopic equations, the densities of EM force and torque as well as those of linear and angular momenta. In the present section, we rely once again on the Einstein-Laub tensor to elucidate certain properties of EM angular momentum, whose density is given by $\mathcal{L}(\boldsymbol{r}, t)=\boldsymbol{r} \times \boldsymbol{p}(\boldsymbol{r}, t)$. Here $\boldsymbol{p}=\boldsymbol{S}(\boldsymbol{r}, t) / c^{2}$ is the EM linear momentum density, $\boldsymbol{S}=\boldsymbol{E} \times \boldsymbol{H}$ is the Poynting vector, and $c$ is the speed of light in vacuum. Below we discuss two examples of EM angular momentum, one in a static situation, the other in a dynamic context, thus highlighting the universality of the concept.

Example 1: shown in figure 5(a) is a uniformly charged hollow sphere of radius $R$ and surface charge density $\sigma_{\mathrm{s}}$. The sphere is spinning at a constant angular velocity $\Omega_{0}$ around the $z$-axis. The total charge of the sphere is $Q=4 \pi R^{2} \sigma_{\mathrm{s}}$, while its magnetic dipole moment is $\boldsymbol{m}=\left(4 \pi R^{3} / 3\right)\left(\mu_{0} \sigma_{\mathrm{s}} R \Omega_{0}\right) \hat{z}$. Inside the sphere the electric and magnetic fields are $\boldsymbol{E}(\boldsymbol{r})=0$ and $\boldsymbol{H}(\boldsymbol{r})=\frac{2}{3}\left(\sigma_{\mathrm{s}} R \Omega_{0}\right) \hat{z}$, whereas outside the sphere,

$$
\begin{gathered}
\mathbf{E}(\mathbf{r})=\sigma_{\mathrm{s}} R^{2} \hat{\mathbf{r}} /\left(\varepsilon_{0} r^{2}\right) . \\
\boldsymbol{H}(\boldsymbol{r})=\frac{1}{3}\left(\sigma_{\mathrm{s}} R^{4} \Omega_{0}\right)(2 \cos \theta \hat{\boldsymbol{r}}+\sin \theta \hat{\boldsymbol{\theta}}) / r^{3} .
\end{gathered}
$$

Integrating the angular momentum density $\mathcal{L}(\boldsymbol{r})$ over the entire space yields the total EM angular momentum of the spinning charged sphere as $\boldsymbol{L}=Q \boldsymbol{m} /(6 \pi R)$. For electrons, $Q=-1.6 \times 10^{-19} \mathrm{C}, m_{z}=\mu_{0} \mu_{B}=-4 \pi \times 10^{-7} \times 9.27 \times$ $10^{-24} \mathrm{~J} \mathrm{~m} \mathrm{~A}^{-1}$, and $L_{z}=\frac{1}{2} \hbar=0.527 \times 10^{-34} \mathrm{~J}$ s. If the electron were a ball of radius $R \cong 1.876 \mathrm{fm}$, its entire angular momentum would have been electromagnetic.

Next, consider figure 5(b), where a second uniformly charged spherical shell of radius $R_{0}$ carrying a total charge of $-Q$ is placed at the centre of the spinning sphere. The $E$-field will now be confined to the region between the two spheres, and the total EM angular momentum of the system is readily found to be

$$
\boldsymbol{L}=\frac{8 \pi}{9}\left(\mu_{0} R^{3} \sigma_{s}^{2} \Omega_{0}\right)\left(R^{2}-R_{0}^{2}\right) \hat{z} .
$$

It is thus seen that, in the limit when $R_{0} \rightarrow 0$, the two angular momenta, calculated with and without the second
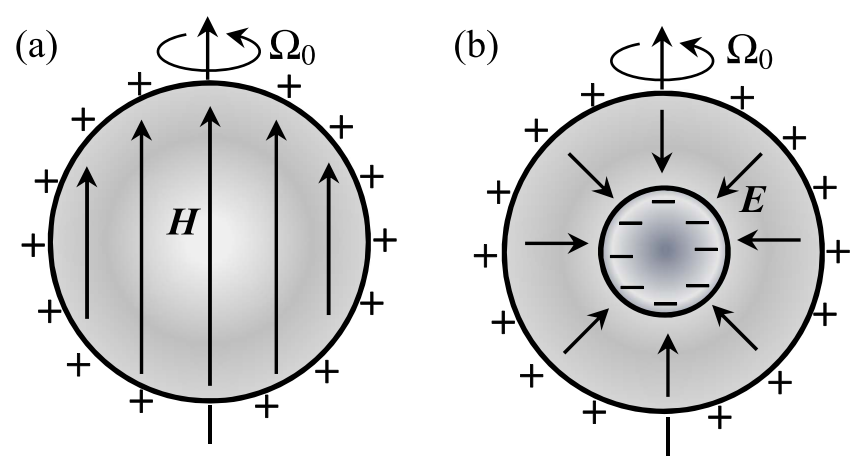

Figure 5. (a) Uniformly charged hollow sphere of radius $R$ spinning at constant angular velocity $\Omega_{0}$ around the $z$-axis. (b) Same as (a), except for the presence of a stationary charged sphere of radius $R_{0}<R$ inside the spinning sphere.

sphere, are identical. In one case, the 'particle' has a net charge of $Q$ and its EM angular momentum is distributed outside the sphere of radius $R$. In the other case the 'particle' (consisting of two spheres) is charge neutral, yet continues to possess a magnetic dipole moment (because the external sphere is spinning). In the latter case, the EM angular momentum is confined to the space between the two spheres.

Example 2: here we consider a single-mode vector spherical harmonic EM wave [48] trapped inside a hollow, perfectly conducting sphere of radius $R$. Choosing the mode's polarization state to be transverse electric (TE) and denoting its frequency by $\omega$, its wavenumber by $k_{0}=\omega / c$, and its polar and azimuthal orders by $(\ell, m)$, the field distribution inside the sphere will be

$$
\begin{aligned}
\mathbf{E}(\mathbf{r}, t)= & E_{0} \frac{J_{\ell+1 / 2}\left(k_{0} r\right)}{\sqrt{k_{0} r}}\left\{\frac{P_{\ell}^{m}(\cos \theta)}{\sin \theta} \cos (m \varphi-\omega t) \hat{\theta}\right. \\
& \left.+\frac{\sin \theta P_{\ell}^{\prime m}(\cos \theta)}{m} \sin (m \varphi-\omega t) \hat{\varphi}\right\} . \\
\boldsymbol{H}(\boldsymbol{r}, t)= & -\frac{E_{0}}{Z_{0}}\left\{\frac{\ell(\ell+1) J_{\ell+1 / 2}\left(k_{0} r\right)}{\left(k_{0} r\right)^{3 / 2}} \frac{P_{\ell}^{m}(\cos \theta) \cos (m \varphi-\omega t)}{m} \hat{\boldsymbol{r}}\right. \\
& -\frac{k_{0} r J_{\ell+1 / 2}^{\prime}\left(k_{0} r\right)+\frac{1}{2} J_{\ell+1 / 2}\left(k_{0} r\right)}{\left(k_{0} r\right)^{3 / 2}} \\
& \times\left[\frac{\sin \theta P_{\ell}^{\prime m}(\cos \theta) \cos (m \varphi-\omega t)}{m} \hat{\boldsymbol{\theta}}\right. \\
& \left.\left.+\frac{P_{\ell}^{m}(\cos \theta) \sin (m \varphi-\omega t)}{\sin \theta} \hat{\varphi}\right]\right\} .
\end{aligned}
$$

In the above equations, $E_{0}$ is the $E$-field amplitude, $E_{0} / Z_{0}$ is the $H$-field amplitude, $Z_{0}=\sqrt{\mu_{0} / \varepsilon_{0}}$ is the impedance of free space, $J_{\ell+1 / 2}(\rho)$ is a Bessel function of the first kind, half-integer order, $\ell+\frac{1}{2}, P_{\ell}^{m}(\zeta)$, is an associated Legendre function of order $(\ell, m)$, and the integers $\ell$ and $1 \leqslant m \leqslant \ell$ are the polar and azimuthal orders of the mode. The primes over $J_{\ell+\frac{1}{2}}(\rho)$ and $P_{\ell}^{m}(\zeta)$ indicate differentiation with respect to $\rho$ and $\zeta$. Since the tangential components of $\boldsymbol{E}$ and the perpendicular component of $\boldsymbol{B}=\mu_{0} \boldsymbol{H}$ must vanish on the internal surface of the perfect conductor, it is necessary that $k_{0} R$ be a zero of $J_{\ell+1 / 2}(\rho)$. 
The field energy density, $\mathcal{E}(\boldsymbol{r}, t)=\frac{1}{2} \varepsilon_{0} E^{2}+\frac{1}{2} \mu_{0} H^{2}$, when integrated over the spherical volume of radius $R$, yields the total energy $\mathcal{E}_{\text {total }}^{(\mathrm{EM})}$ of the mode trapped inside the cavity. In the limit of large $R$ we find

$$
\mathcal{E}_{\text {total }}^{\text {(EM) }} \approx\left(\frac{R E_{0}^{2}}{\mu_{0} \omega^{2}}\right) \frac{\ell(\ell+1)(\ell+m) !}{m^{2}\left(\ell+\frac{1}{2}\right)(\ell-m) !} .
$$

Similarly, integration of the angular momentum density $\mathcal{L}(\boldsymbol{r}, t)$ over the sphere's volume in the limit of large $R$ yields

$$
L_{z}^{(\mathrm{EM})} \approx\left(\frac{R E_{0}^{2}}{\mu_{0} \omega^{3}}\right) \frac{\ell(\ell+1)(\ell+m) !}{m\left(\ell+\frac{1}{2}\right)(\ell-m) !} .
$$

Thus, for a sufficiently large sphere, the ratio of OAM to EM energy is seen to be

$$
L_{z}^{(\mathrm{EM})} / \mathcal{E}_{\text {total }}^{(\mathrm{EM})}=m / \omega .
$$

This is consistent with the quantum-optical picture of photons of energy $\hbar \omega$ and OAM $m \hbar \hat{z}$ being trapped within the cavity.

\section{Angular momentum conservation}

Returning to the system of figure 5(a), let the sphere's angular velocity be a function of time, $\Omega(t)$, which starts at zero and rises slowly to $\Omega_{0}$ after a long time. In accordance with Faraday's law $(\boldsymbol{\nabla} \times \boldsymbol{E}=-\partial \boldsymbol{B} / \partial t)$, the changing magnetic field inside the sphere induces the $E$-field $\boldsymbol{E}(\boldsymbol{r}, t)=-\frac{1}{3} \mu_{0} \sigma_{\mathrm{s}} R r \sin \theta \Omega^{\prime}(t) \hat{\boldsymbol{\varphi}}$. The torque acting on the spinning sphere by this $E$-field will then be

$$
\begin{aligned}
\boldsymbol{T}(t) & =\int_{\theta=0}^{\pi} \int_{\varphi=0}^{2 \pi} \boldsymbol{r} \times \sigma_{\mathrm{s}} \boldsymbol{E}(R, \theta, \varphi, t) R^{2} \sin \theta \mathrm{d} \theta \mathrm{d} \varphi \\
& =-\frac{8 \pi}{9} \mu_{0} R^{5} \sigma_{\mathrm{s}}^{2} \Omega^{\prime}(t) \hat{z} .
\end{aligned}
$$

Integration over time now reveals that the mechanical angular momentum delivered by an external torque during the spin-up process is precisely equal to the EM angular momentum $\boldsymbol{L}$ residing in the space surrounding the spinning sphere of figure 5(a). A similar calculation shows that the EM angular momentum in the system of figure 5(b) is fully accounted for by the mechanical torque acting on the outer (spinning) sphere minus the torque needed to keep the inner sphere stationary.

The above argument, although employed here in conjunction with simple quasi-static systems involving slowly spinning charged spheres, applies equally well to more complicated dynamic situations $[43,49]$. In general, any change in the EM angular momentum of a system is always accompanied by a corresponding mechanical torque acting on the material bodies of the system. The existence of this torque ensures that any change in the EM angular momentum is compensated, precisely and instantaneously, by an equal and opposite change in the overall mechanical angular momentum of the system [50].

\section{Current and future challenges}

To date, essentially all experimental research on EM angular momentum has been carried out with non-magnetic materials. Since some of the major differences among the various EM stress tensors depend on magnetization, it will be worthwhile to explore the mechanical response of magnetized (or magnetizable) media in the presence of EM fields, in particular, external electric fields, which give rise to the so-called 'hidden momentum' in conjunction with the standard Lorentz stress tensor [51]. A topic of fundamental importance is the distribution of EM force density and/or torque density within material objects, which can only be monitored in soft (i.e. deformable) matter. Although different stress tensors might predict identical behaviour for a rigid object in response to the overall EM force/torque acting on the object, local deformations of soft media are expected to vary depending on the distribution of force and torque densities throughout the object. Precise measurements of this type can thus distinguish among the various stress tensors that have been proposed in the literature.

Another interesting topic is the interaction of light that carries EM angular momentum with rotating bodies such as mirrors, transparent or partially absorbing plates, and birefringent media [52, 53]. This would involve exchanges of angular momentum as well as energy, resulting in a Doppler shift of the emerging photons.

\section{Advances in science and technology to meet challenges}

The most dramatic effects of EM linear and angular momenta arise in conjunction with high-quality-factor microresonators and nanodevices. Advances in micro- and nanofabrication in tandem with development of novel mechanisms for coupling light and/or microwaves into and out of high- $Q$ cavities will enable sensitive measurements of optomechanical interactions. Development of high-quality transparent magnetic materials as well as novel metamaterials with controlled permittivity, permeability, dispersion, birefringence, nonlinear coefficients, etc., will usher in not only a deeper understanding of the fundamentals but also a treasure trove of new applications.

\section{Concluding remarks}

While the classical theory of EM angular momentum is well established, there remains opportunities for theoretical contributions to the understanding of nonlinear phenomena, quantum optics of non-paraxial beams, and interactions between light and rotating objects. On the experimental side, one could look forward to precise measurements of EM force density and torque density distributions in conjunction with deformable media in order to identify appropriate stress tensors for ordinary matter and also for exotic metamaterials. The bidirectional exchange of angular momentum between EM fields and material objects continues to provide a rich and rewarding platform for exploring novel practical applications. 


\section{Shaping light}

\section{Cornelia Denz and Christina Alpmann}

University of Muenster

\section{Status}

Since Theodore Maiman invented the laser in 1960 it has become an indispensable tool in our daily lives. Its unique properties as, for example, the ability to achieve high power while being focused to a diffraction-limited spot, makes the laser ideal as a precision scalpel in medicine or as a means to cut or join plates of steel. The key feature of lasers is that the amplified light is well defined and reproducible and can be tailored in terms of intensity and phase, propagation properties or polarization. Therefore, beam shaping has been a field of interest since the invention of the laser and numerous modulation techniques for light structuring in and outside a laser cavity have been developed. Even before the invention of the laser, Dennis Gabor established the first method of imprinting amplitude as well as phase information of light by the development of holographic imaging referred to as holography, for which he was honoured by the Nobel Prize in Physics in 1971 [54]. Since then, holographic methods spread in many ways and technological developments enabled numerous applications, even prominent in daily life as, e.g., the safety features of bank notes. Subsequently, beam shaping paved the way to principle implementations such as diffractive optical elements: computer-calculated holograms that are afterwards transferred onto holographic plates. Their development and acceptance was mainly due to modern lithography techniques and digital hologram calculation [55], and allowed for 2D as well as 3D structuring of light, though being static. The ultimate breakthrough of holographic modulation techniques arose from the invention of digital SLMs in the form of liquid crystal displays originally developed for video projection $[56,57]$. Nowadays, such devices allow for real-time wavefront shaping by computer-generated holograms without any intermediate material or production step, and enable desktop creation of customized light fields. In modern optics, spatial modes represent a current topic with highest importance for numerous areas in optics such as, optical communication technology, quantum optics, data security in information optics, or optical manipulation and its application in life sciences.

\section{Current and future challenges}

Today, structured light represents an established division of the broader field of modern optics and photonics. Originating from higher-order modes in laser resonators such as Laguerre-Gaussian and Hermite-Gaussian beams, several classes of complex light shapes have become experimental reality, and are successfully applied in many fields of optics. A glimpse of the manifold of 2D and 3D light fields is given in figure 6, including standard as well as elegant Gaussian beams, nondiffracting beams, accelerating light fields,

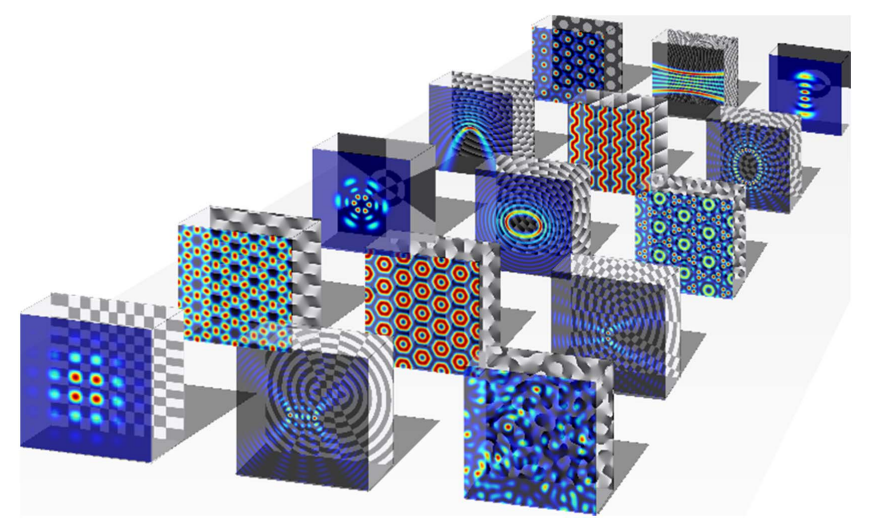

Figure 6. Overview of 15 exemplary, structured light fields with corresponding amplitude (front) and phase (back) distributions, respectively.

(quasi-) periodic via aperiodic up to random tessellation structures. Thus, the emerging field of structured light bears challenges in the future that are strongly connected to applications of tailored light shapes and associated innovations.

In quantum optics, spatially shaped modes carrying OAM, such as, e.g., Laguerre-Gaussian or Bessel modes, represent a higher-dimensional orthogonal basis of qubits and are assessed with respect to the entanglement of OAM (see sections 17 and 18) [58], with its tremendous potential for data encryption and security. Beyond that, spatial modulation of polarization states becomes more and more important (see sections 7 and 8) [59]. While primarily interferometric methods and so-called q-plates allow the generation of radially and azimuthally polarized beams, liquid crystal SLMs have pioneered the realization of extended Poincaré beams. Such recent developments in holographic spatial polarization shaping facilitate prospective studies on singular optics and classical entanglement. Figure 7 shows a variety of singular polarization structures with the future impact available with such techniques.

Another area where structured light fields play a prominent role with ever increasing impact is life sciences. The spatial resolution of multiple microscopy techniques such as, e.g., STED or light sheet microscopy depends on accurate shaping of respective light distributions and may advance considerably with the progression of such techniques (see section 10). Furthermore, in optical manipulation (see sections 15 and 16), tailored light modes meet the strong demand for extended optical potential landscapes that allow transfer to the micro- and nanostructures of particles [60-62], or unveiling the biophysical origins of cell dynamics and cell interactions in organisms. Consequently, holographic shaping techniques are driving the innovation of custom-designed, tailored complex light fields and have become essential for new developments across disciplines from classical and information physics, and quantum physics, to biophysics, medical diagnosis and therapy. Numerous applications in photonics as well as interdisciplinary technologies, including opto-electronics, opto-fluidics, biophotonics, photonic crystals, and metamaterials are based on a plethora of discoveries 


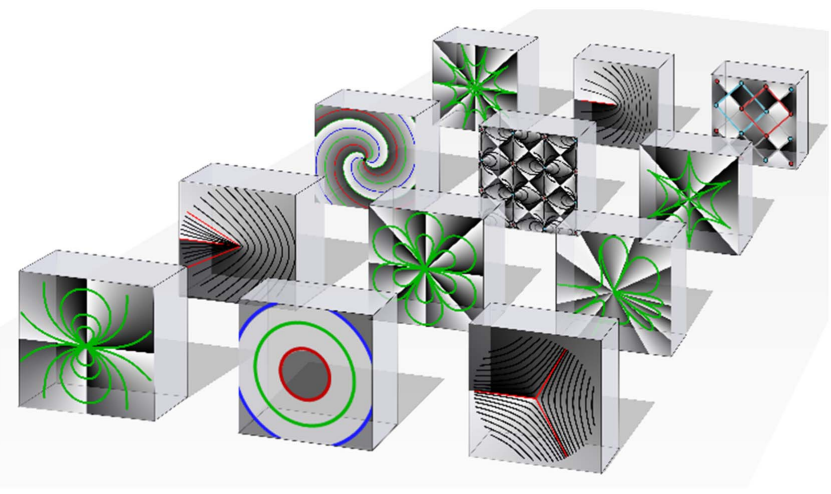

Figure 7. Overview of 12 different polarization singular structures visualized by the corresponding Stokes field phase (back) and sketches of flow and singular lines and points (front).

and technical milestones enabled by spatial shaping of amplitude, phase, and, only recently, polarization properties of light.

\section{Advances in science and technology to meet challenges}

Wavefront shaping has become an important ingredient of many cutting-edge optics and photonics fields. In optical communication technologies, data transfer has been advancing rapidly for several decades supporting increasingly information-driven society. In addition to multiplexing in time and frequency, wavelength, polarization and phase, the spatial dimension has been exploited by mode multiplexing only recently [63]. Therefore, light shaping will be one solution to improve the exploitation of existing capacities. Aside from an increase in capacities and transfer rates, future information technology needs to come up with novel techniques of data encryption and security where structured light fields will also take over an important role. The high potential of spatial light shaping in amplitude, phase, and polarization enables combinations of singular structures providing additional properties and parameters. As singularities do not only occur in optics but in several physical systems such as magnetic or solid state physics, results obtained in singular optics are of general interest.

As an important prerequisite for further technological innovations, novel developments of light shaping techniques need to become more visible and more easily available to a broad community. As a promising advance in reducing acquisition times in scanning microscopy, direct laser writing or other laser cutting, fusion or manipulation techniques would be the implementation of complex lattice beams for parallel processing. Consequently, the exploration of advanced holographic modulation techniques for light field shaping, which enables tailoring light to match the continuous increase of complexity of applications, is a remaining future challenge.

From a technological point of view, further development of digital SLMs is required where efficiency, spatial homogeneity, and especially spectral bandwidth and speed are current limitations. While efficiency and spatial homogeneity are technical challenges that are currently already under development and will improve continuously over the next few years [64], e.g. by developing LCOS (liquid crystals on silicon) based SLMs further, a major remaining challenge is the control of structured light in the UV domain and up to the $\mathrm{X}$-ray regime. Beneath developments of innovative SLMs for this wavelengths regime, novel approaches including frequency conversion of shaped light to access wavelengths regions that cannot otherwise be accessed by direct modulation are actually being developed [65]. Once available, these light shaping techniques will allow the enlargement of the impact of structured light being employed in the future in many trans sectoral areas, such as, e.g., to enhance the plasmon excitation of metallic nano-antennas by tailored illumination, showing high innovation potential and the outreach of shaped light.

\section{Concluding remarks}

Based on the early work of Gabor, holographic light shaping developed into a flexible and powerful research direction in modern optics. It primarily started as a self-contained field, and continuous development over the past decade has led to an imminent, interdisciplinary breakthrough. We believe that if holographic modulation of amplitude and phase, as well as polarization, is further developed, and interdisciplinary projects are fostered where tailored light fields represent an enabling technology for scientific advances as well as technical devices, a plethora of new applications will arise. Possibly this will happen in a two-step process initiated by phase and amplitude shaping and followed, or even passed, by spatial polarization structures. We are convinced that shaping light is an innovation-driving field with excellent future prospects.

\section{Acknowledgments}

The authors thank M Boguslawski and E Otte for support in figure preparation. 


\section{Structured polarization and its measurement at the nanoscale}

\section{Peter Banzer and Thomas Bauer}

Max Planck Institute for the Science of Light

\section{Status}

The polarization degree of freedom of light allows for a wide range of applications in microscopy, optical communications and many other fields of optics. While in the most fundamental case the polarization in the transverse profile of a light beam propagating paraxially is homogeneous, it may also exhibit a spatially nonhomogeneous and complex distribution (see figure 8(a), right; see also sections 6 and 8). For the generation of such modes, spatially engineered optical elements acting on the local polarization of a homogeneously polarized input beam can be utilized. Various concepts based on liquid crystal cells [14, 66, 67] as well as segmented waveplates [68] have been discussed in the literature (see sections 6 and 8). The polarization characteristics and distributions of the resulting modes can be measured easily by performing a spatially resolved Stokes analysis, since the electric field vector is restricted to an oscillation perpendicular (transverse) to the propagation direction of the beam in the paraxial limit. This is not necessarily the case if light is tightly focused (see figure 8(b)). In the field distribution created upon focusing a light beam down to dimensions of the order of the wavelength, the electric field needs a 3D description. Because of the occurrence of large transverse wavevectors, longitudinal oscillations of the field vectors, i.e. along the propagation direction, can also be observed. This appearance of longitudinal field components has striking consequences. For instance, it allows for focusing light to a tighter spot [68] or it may result in complex polarization topologies [69]. Furthermore, shaping the input beam enables the tailoring of the 3D focal field and polarization distribution. Unfortunately, conventional approaches designed for measuring paraxial light beams are not applicable for mapping these focal fields, due to the nanoscale features and the complex 3D nature of the distributions under study.

For the measurement of selected polarization components within highly confined fields, the use of individual fluorescent molecules with a fixed dipole axis was proposed and demonstrated (figure 9(a) and [70]). Later, it was shown that wellcharacterized near-field tips, as used for near-field scanning optical microscopy (NSOM), can be utilized to map confined fields and to distinguish between individual polarization components. With this technique even the spatial phase distributions of the electric field components were measured using an interferometric detection approach (figure 9(b) and [71]).

More recently, a method for experimentally reconstructing the fully vectorial information of a focused light beam was presented [72]. Here, an individual nanoparticle is utilized as the scanning field-probe. The field scattered off the nanoparticle may interfere with the impinging light beam under study. The information about the local relative phase of (a)
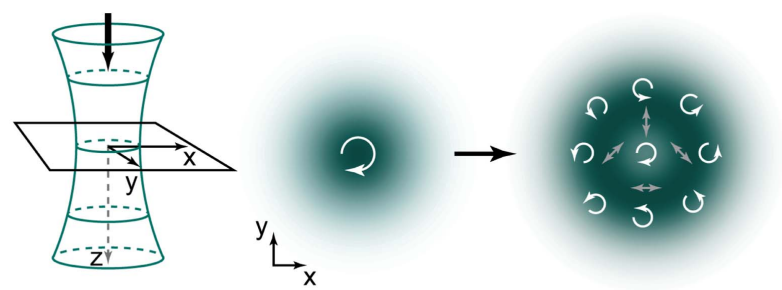

(b)
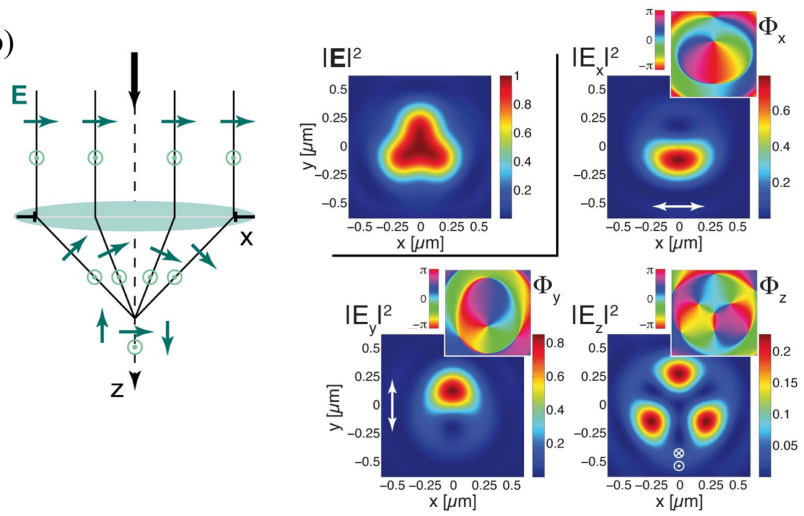

Figure 8. (a) In paraxial light fields, the lateral polarization structure can be transformed from homogeneous to inhomogeneous distributions. (b) Sketch of tight focusing of polarized light beams (left), resulting in complex focal field distributions (right, from [69]. Reprinted with permission from AAAS). (a)

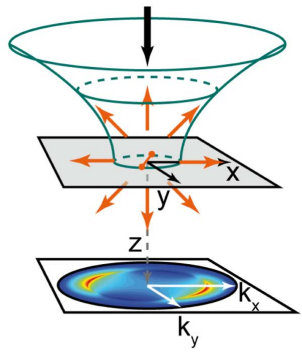

(b)

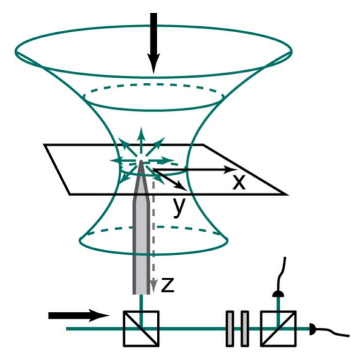

(c)

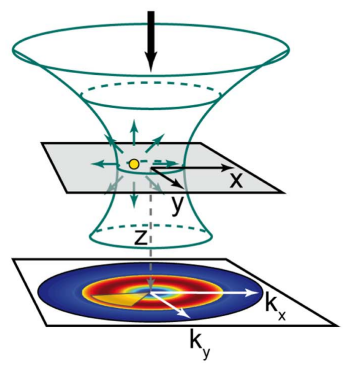

Figure 9. Selected schemes for the measurement of highly confined polarization distributions. (a) Direct mapping of individual field components using single fluorescence molecules. (b) Coherent measurement of field components by NSOM. (c) Full field reconstruction with a single scattering nanoprobe and angularly resolved far-field detection of the transmission signal.

the electric field components is stored in this interference term. This allows for the reconstruction of amplitude and phase distributions of the focal field from an angularly resolved measurement of the intensity in the far-field (see figure 9(c) and [72]). With this approach, the experimental study of highly complex focal fields with unprecedented resolution became possible [69]. 


\section{Current and future challenges}

One of the most important prerequisites for both NSOM and particle-based field measurements at the nanoscale as described above is the precise knowledge of the probe geometry and its optical response at the chosen wavelength of the field distribution under investigation. This is a major problem for quantitative NSOM measurements of field distributions because not only the near-field tip itself but also the attached tapered fibres influence the field patterns and, therefore, both have to be well characterized. The particle-based approach, by contrast, requires the knowledge of the particle only, since it is combined with a rigorous description of the interaction of the particle with the focal field and its back-action. Hence, this technique is not restricted to the use of comparatively small and spherical nanoprobe designs as demonstrated, exhibiting a dominantly dipolar scattering response. If the geometry, dimensions and optical properties of the material are well known, almost arbitrarily shaped nanostructures can be used for this reconstruction method. Nonetheless, this technique is so far still limited to the investigation of propagating fields, because it requires an inline reference for the determination of the relative phases. An extension to nonpropagating evanescent fields is conceivable by adapting the post-processing algorithms. Another drawback is the necessity of using a substrate as a support for the particle probe, so far also limiting the technique's applicability to the case of focused light. By contrast, NSOM can be used for measuring the polarization structures even in the near-fields around nanoscale scatterers or waveguides-a simple consequence of the more accessible probe geometry. This important feature would be highly desirable for particle-based approaches.

Because the response of the probes has to be known precisely, both concepts are normally applied for spectrally narrowband configurations only. To enable, for instance the experimental study of spectrally broad beams or pulses under tight focusing conditions, knowledge of the full spectral response of the field probes is thus essential. For this specific scenario, tailoring or shaping the probe geometry to influence its spectrally dependent interaction with the light field will be of great benefit.

\section{Advances in science and technology to meet challenges}

An important step towards this detailed spectral probe characterization can again be realized with the help of spatial field distributions tailored at the nanoscale level. In the aforementioned reconstruction technique, a well-characterized nanoparticle is used to probe an unknown field. The inversion of this idea-the utilization of a well-characterized focal field distribution for the study of individual nanostructures-paves the way for the detailed spectral analysis of an individual particle's multipolar spectral response (see [73] and references therein). Different spatial field distributions are utilized for the selective excitation of an individual nanoparticle, enabling the detailed study of its spectral response. To be able to understand the dynamics in the interaction between spatially and temporally tailored and confined light fields with such a nanoparticle, its nonlinear response may also play a major role. In this context, first experimental studies reveal the potential of this nonlinear interaction of individual particles with tailored light to learn, for instance, about the particle's shape [74]. An extension of this and other related concepts discussed already in the literature may also allow for the precise and spectrally resolved determination of the linear and nonlinear optical response of individual nanoparticles. At the same time, a more detailed theoretical and numerical investigation of the temporal dynamics of tightly focused and spatially structured light pulses is required.

In combination with the technological advances in the fabrication of high-quality nanostructures, the above-mentioned experimental tools for the characterization of field probes and adequate extensions of field reconstruction techniques will also allow for the experimental study of the dynamics of optical pulses propagating non-paraxially. This understanding of complex light-matter interactions will open up new avenues for the development of novel technologies, enabling the management, guidance and control of light at nanoscale dimensions.

\section{Concluding remarks}

Confined field distributions in general and complex spatial modes of light, in particular, have been proven to be extremely versatile and flexible tools at the nanoscale (see, for instance, $[70,73,74]$ and references therein). As such, they are an invaluable extension of the optical toolbox and find a wide range of applications in optics and related fields of research. In addition, polarization tailoring and strong confinement of electromagnetic fields may also give rise to intriguing effects and phenomena (see $[68,69]$ and references therein). To facilitate the experimental study of such effects and to characterize tightly focused or confined fields to be used as tools in nanooptical applications, their experimental investigation with a spatial resolution far below the wavelength is essential. In this context, both techniques described above-near-field microscopy [71] as well as particle-based nanointerferometry [72] - are excellent choices for measuring the full field information at the nanoscale, with a great potential for further developments.

\section{Acknowledgments}

The authors thank Gerd Leuchs for fruitful discussions. 


\section{Space-varying polarized fields: classical and quantum prospects}

\author{
Ebrahim Karimi ${ }^{1}$ and Lorenzo Marrucci \\ ${ }^{1}$ University of Ottawa \\ ${ }^{2}$ Università di Napoli Federico II
}

\section{Status}

In most optical experiments and applications, the polarization across the light beam is uniform, or at least approximately so. The possibility of having space-varying polarization across the beam was obviously there, but until the early 2000s there was no particular interest in such 'exotic' beams. This, however, changed when it was first realized that radially and azimuthally polarized beams exhibit very intriguing properties upon tight focusing [68]. In the focal region, they respectively generate 'needle' electric and magnetic fields oriented along the beam longitudinal propagation direction and with a beam waist that is smaller than for a standard Gaussian beam. This important result is of obvious interest for applications such as optical nano-probing, nano-lithography or high-density optical memories. Radially and azimuthally polarized beams are specific examples of so-called cylindrical vector $(\mathrm{CV})$ beams, i.e. beams having a cylindrically symmetric polarization pattern, and of the even more general class of vector vortex (VV) beams, i.e. beams with an azimuthally varying linear polarization surrounding an optical vortex located on the beam axis (see figures 10(a) and (b)) [75]. VV beams are the most general light beams having propagation-invariant transverse polarization patterns (except for diffraction-induced radial rescaling). They have recently attracted increasing interest for various applications. For example, VV beams approximately match the higher-order eigenmodes of multi-mode optical fibres, currently considered for possible 'mode-division multiplexing' in future optical communication technology. Unlike standard polarized photons, photons of $\mathrm{CV}$ beams carry rotation-invariant qubits of quantum information, useful for establishing a quantum communication link between continuously rotating or randomly oriented parties, as for example in future satellitebased quantum networks or hand-held wireless quantum devices [76]. Conversely, high-order VV beams can have the property of being highly sensitive to rotations, and this in turn can be used to measure tiny mechanical rotations.

Another important family of light fields is that of the 'Poincaré beams' [77], which are light beams exhibiting all possible polarization states in their cross-section and having a polarization singularity on their axis. This singularity is not an optical vortex in a standard sense, e.g., there is no vanishing of the fields at its core, although it is associated with a certain degree of 'vorticity'; in certain scientific communities, it is also named 'half-vortex', because the optical phase changes by $\pi$ when circling once around the singularity (but the wave remains continuous and single-valued thanks to the polarization variation). Polarization singularities [2], spontaneously arising in any pattern of light with random
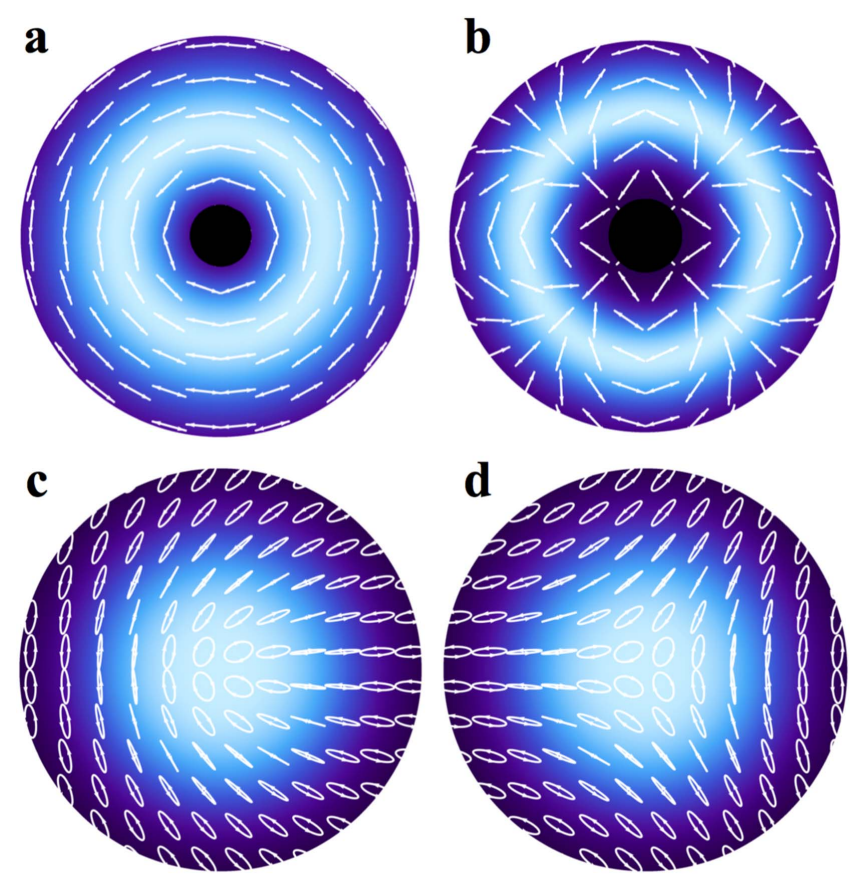

Figure 10. (a) and (b) are examples of vector vortex beams; (a) in particular is an azimuthally polarized beam. (c) and (d) are Poincaré beams having 'lemon' and 'star' polarization topologies, respectively. Colour brightness represents the intensity distribution, while the local polarization is depicted as small white ellipses or lines.

polarization, are points having circular polarization ( $\mathrm{C}$ points) or linear polarization (L lines), surrounded by generic elliptical polarizations. The lowest-order C-point polarization singularities have been classified according to the topology of the surrounding polarization ellipse orientations (see, e.g., figures $10(\mathrm{c})$ and (d)). Unlike VV beams, the polarization pattern of Poincaré beams changes upon propagation, but following simple rules (e.g., it rotates around the beam axis).

In the quantum regime, photons with space-varying polarization can also be described as nonseparable ('entangled') quantum states of the polarization and spatial degrees of freedom. Such a form of single-particle entanglement, although not challenging local realism, can still be used for certain quantum information protocols [78].

\section{Current and future challenges}

A major current challenge in this area is that of moving from 2D field polarization structures to 3D cases. 3D here means exploiting all three field components, rather than the sole transverse ones, but also being able to generate a field that is not only structured within the transverse plane but also along the longitudinal coordinate, as in time-varying polarization pulses. Some first steps in creating complex 3D field structures in terms of field components have been taken recently. These have relied on the fact that the longitudinal field component is non-negligible for a non-paraxial beam. In this generalized case, the polarization ellipse is not transverse and defines a plane of oscillation that is spatially varying in 3D. Isaac Freund first predicted the existence of $3 \mathrm{D}$ polarization topologies in the form of multi-twist Möbius strips and 

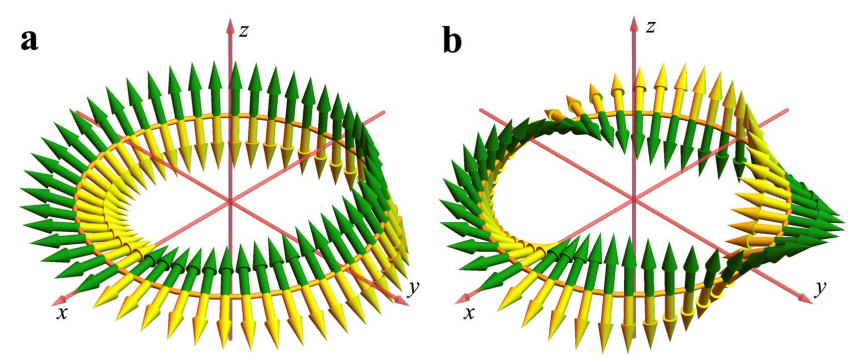

Figure 11. Examples of 3D polarization topologies in the form of Möbius strips with half and three-half twists are shown in (a) and (b), respectively. Represented is the direction of the polarization ellipse major axis as a function of position around the beam axis.

twisted ribbons for spatially structured optical beams [79]. Such 3D structures have recently been observed experimentally in the focal volume of a tightly focused Poincaré beam (see figure 11) [71]. However, these are just the first examples, and many other complex 3D polarization structures could be addressed in the future.

Pulsed vector fields having space-varying polarization along the propagation have already been demonstrated, using Gouy phase effects, self-phase modulation and pulse 'chirps'. However, combining such methods with a transverse patterning, so as to achieve full $3 \mathrm{D}$ vector field structuring, has not been attempted yet and is a major future challenge. Another possible important challenge is that of extending the polarization structuring ability to other spectral domains, such as terahertz or extreme ultraviolet.

In the quantum regime, the main current challenge is that of developing more efficient and controlled methods for generating and measuring complex entangled and hyperentangled states involving multiple photons and using both polarization and spatial degrees of freedom for the entanglement. A more specific challenge is also that of using rotationinvariant photons for efficient quantum communication at long distances, against the limiting effects of air turbulence and beam divergence.

\section{Advances in science and technology to meet challenges}

The required technical advances concern, of course, the generation, but also the manipulation and characterization of the generated fields or photons. Current methods for generating standard 2D vector fields exploit holography (with SLMs) or 2D-patterned anisotropic media (e.g., liquid crystal q-plates and plasmonic metasurfaces) [80, 81]. To add a longitudinal field component, one then sends the light through high-numerical aperture (NA) optics, such as a microscope objective [71]. However, this approach is clearly limited in terms of possible outcomes. So, a more general non-paraxial optics will have to be developed in order to fully control the $3 \mathrm{D}$ field structures to be generated. For the generation of 3D pulsed vector light, a suitable combination of the current $2 \mathrm{D}$ structuring techniques with the spectrally resolved methods used to control the pulse shape will probably need to be developed.

The greatest technical problem in all cases will be that of proper characterization of the generated fields. For the 3D field structures obtained in the focus of high NA optics, a technique based on angle-resolved light scattering from a nanoparticle that is raster-scanned in the plane (or volume) of study has recently been developed to measure the amplitude and phase of all three individual components of the electric field (see section 7 and [71]). However, the need for other techniques, such as the equivalent of $3 \mathrm{D}$ photo imaging, remains strong. More generally, there are calls for ultrafast, efficient and possibly miniaturized devices capable of generating and sorting structured beams for all possible applications. In the ongoing race to develop novel optical materials and systems, possible solutions could be based on patterned electro-optics and ultra-thin plasmonic devices, respectively capable of rendering fast-modulation and detection, as well as integration.

\section{Concluding remarks}

The recent advances in the generation, manipulation, and reconstruction of optical fields with complex polarization topologies have allowed the ideation and proof-of-principle demonstration of many interesting applications, such as highresolution optical writing and reading, high-bandwidth or ultra-secure communication, and optical metrology. More potential applications will emerge if it is possible to overcome the challenges discussed here. 3D-structured vector fields may for example provide an innovative platform for sub-wavelength lithography of complex structures, or allow optimized coupling to specific material excitations having similar topologies. However, much remains to be done to acquire full control of the vectorial nature of light.

\section{Acknowledgments}

Ebrahim Karimi acknowledges the support of Canada Research Chairs (CRC) and Canada Excellence Research Chairs (CERC) programs. Lorenzo Marrucci acknowledges the support of the European Research Council (ERC), under grant no. 694683 (PHOSPhOR). 


\section{Imaging with structured light}

\section{Miles Padgett}

University of Glasgow

\section{Status}

Structured light is widely used in conjunction with standard camera systems to reconstruct the 3D surface profile of objects [82]. Such systems often rely upon the oblique structured illumination and the resulting distortion of the projected pattern introduced by the profile of the surface. Similarly, structured light can also be used to boost the resolution of microscope systems beyond that of the imaging optics [83]. This resolution enhancement is derived from illuminating the object with a light pattern containing high spatial frequencies that, through aliasing, down-convert even higher spatial frequencies in the object such that the highresolution image information is recorded. Beyond structuring the intensity of the illumination, phase-contrast imaging relies upon phase structuring and spatial coherence to give various image enhancement modalities familiar to any microscopist. Most recently, these various modalities can be adapted and interchanged by the incorporation of SLMs into the microscope [84], see section 10.

Rather than covering these established areas, this short review will concentrate on structured illumination in more unusual settings, both in the classical and quantum regimes. As such, this review will outline the principles of ghost imaging that, as we will see, is related to the field of singlepixel cameras.

\section{Current and future challenges}

\section{Ghost imaging and single-pixel cameras}

Conventional camera systems use light that is scattered or transmitted by the object and subsequently imaged. Alternatively, so-called 'ghost imaging' systems can use downconversion sources that produce twin light beams within which the photons are position correlated with each other. One of these light beams then illuminates an object while the image information is recovered from the other beam that, surprisingly, has never itself interacted with the object. The image recovery relies upon the spatial correlation between the photon pairs set by the down-conversion process [85]. Whenever a photon from the illuminating beam is transmitted/reflected by the object then the photon event, but not its position, is recorded. This detection event is correlated with the measured position of the correlated photon in the other beam. Recording the position of these correlated photons summed over many photon pairs reveals the image information even though the position-recorded photons did not interact with the object.

However, spatial correlations also exist between classical light beams, most obviously when a beam imprinted with a spatial pattern is split using a beam splitter. Consequently, ghost imaging can be performed using either thermal or quantum light sources in the many- and single-photon regimes, respectively [86]. For the many-photon case, the reconstruction algorithm is slightly more complicated. One of the patterned beams illuminates the object and the intensity of the transmitted or reflected light is recorded using a single element (i.e. single-pixel) detector. The other beam, containing the same pattern, which never interacts with the object, is imaged by a camera, thereby revealing the shared pattern. Adding together each of these patterns, weighted by the measured intensity, reveals the image, albeit in the manyphoton case this image is superimposed on top of a significant background.

One alternative example of ghost imaging dispenses with the beam splitter and instead uses a programmable SLM to create a known but complicated pattern with which to illuminate the object. Rather than being between two light beams, the spatial correlation is now between the projected pattern and the control of the SLM; hence the term computational ghost imaging [87]. As with conventional ghost imaging, adding together these known patterns multiplied by the corresponding measurements of the transmitted/reflected intensity reveals the image.

These computation ghost imaging systems are time reversible, i.e. the light source and detector can be interchanged. In this reversed configuration the object is illuminated by a conventional light source (or even ambient light) and the single-pixel detector measures the transmitted/ reflected light, but as spatially filtered by the SLM. Such reversed configurations are called single-pixel cameras [88], a field which has developed in parallel with that of ghost imaging (see figure 12).

The image reconstruction algorithms that one can apply to computation ghost imaging and single-pixel cameras are largely the same. Fundamentally, whereas a raster-scanned imaging system measures precisely each pixel, computational imaging systems of the type described here measure the correlation between the whole image and specific pattern, thereby giving partial knowledge of all pixels. For a perfect measurement of the image, the number of orthogonal patterns required is equal to the number of pixels and hence both computational and raster-scanning techniques appear to require the same measurement resource. However, recognition that images are data compressible (cf JPEG) means that even for a subset of patterns and measurements it is possible to obtain high-quality images by constraining the reconstruction to be sparse in, e.g., its spatial frequency domain [89]. Using these compressed sampling techniques coupled with inverse problem solving approaches allows image reconstruction whilst using only a fraction of the measurement resource of a raster-scanned system.

\section{Advances in science and technology to meet challenges}

Ghost imaging is intriguing, and in some cases counterintuitive, is clear. What is not so clear is what imaging 


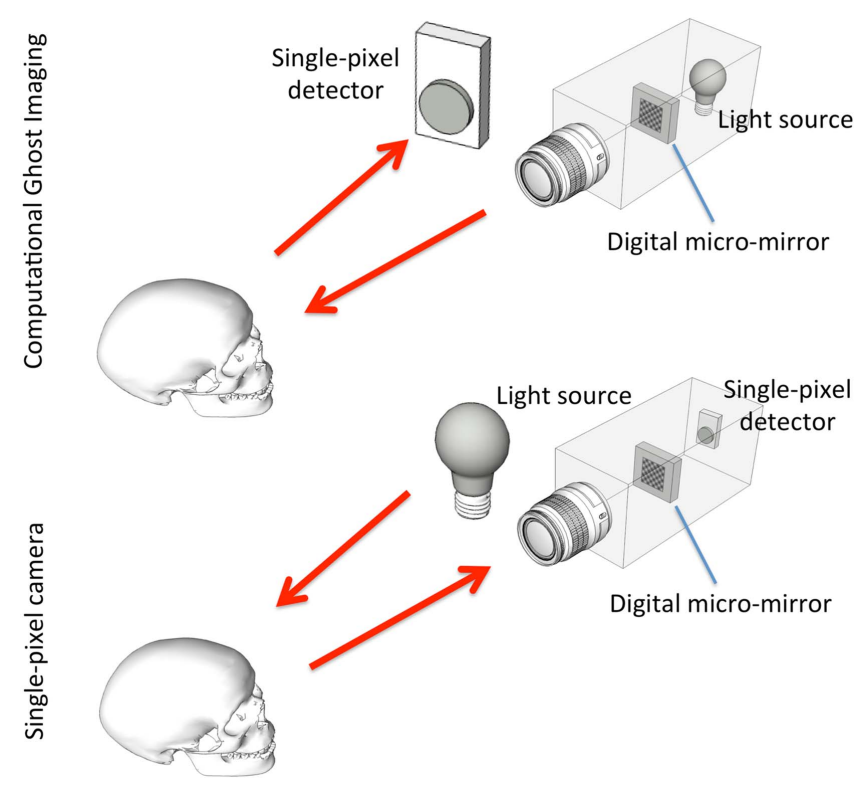

Figure 12. Computational ghost imaging and a single-pixel cameras are in essence very similar.

functionality it uniquely offers. Ghost imaging with correlated photon pairs provides a number of opportunities that would be difficult to match with conventional technologies. Building conventional cameras with true single-photon capability is challenging. Even a small dark-count probability summed over a many-pixel array amounts to a significant number of noise events within a single image and hence the recording of the position of a single photon is obscured. Further reduction of the dark count requires extreme time gating of the detection window, which is exactly what coincident detection allows. True single-photon sensitivity and the elimination of noise allows images to be reconstructed from only a few thousand photons, i.e. fewer than one photon per pixel in the final image [90]. The technical challenge here is for technologies with single-photon performance in both the visible and nonvisible regions of the spectrum.

Another opportunity afforded by imaging based on photon pairs is that, although position correlated, the photon pairs do not need to be the same wavelength. Hence, the object can be illuminated in the infrared yet the image information be recovered from correlations in the visible, a spectral region in which camera performance is a maximum [91]. This photon level transfer of the position information between wavelengths creates an interesting opportunity for photon-by-photon far infrared or even terahertz imaging. Here the technical challenges lie in obtaining very nonfrequency- degenerate photon-pair sources, and, in some other configurations, long-wavelength single-photon detectors.

Moving to single-pixel cameras, their main advantage lies in the removal for any need of a detector array. In the visible region of the spectrum, the ever-improving performance of silicon-based detector arrays means that there is perhaps little need for alternative approaches. However, once outside the silicon accessible spectral detection window, detector arrays are not so straightforward. Within a singlepixel camera, the SLM can take several forms ranging from mechanical masks to liquid crystal and DMDs. DMDs of the type found in many digital image projectors are capable of pattern update rates in excess of $10 \mathrm{kHz}$ and have an operational window extending well beyond the visible spectrum. Similarly, single-pixel detectors based on different material types have spectral sensitivity across many possible wavelengths. Single-pixel cameras can therefore be made covering various spectral ranges with an overall system complexity and cost not exceeding that of a commercial data projector [92].

One challenge for these computational ghost imagers and single-pixel cameras is in the development of SLMs operating far outside the visible spectrum. Another challenge lies in the single-pixel detector itself. Although only a single-pixel it still needs to have a high optical étendue since images are inherently multi-spatial mode and the optical design of the detection optics needs to take this into account. The high étendue requires a detector with an appreciable active area, which invariably leads to an increase in its noise equivalent power.

\section{Concluding remarks}

Imaging is by its very nature a structuring of the light field in either or both intensity and phase. Traditional imaging systems rely upon spatial resolving detectors (e.g. eye, film, CCD) to measure the light transmitted or scattered from an object. However, correlations between photon pairs, classical light beams or light patterns and computer controlled spatial modulators create opportunities to image without detector arrays taking advantage of the wider spectral coverage or time resolution offered by single-pixel detectors.

\section{Acknowledgments}

MP acknowledges the financial support of the European Research Council (advanced grant TWISTS) and the Engineering and Physical Sciences Research Council (QUANTIC). 


\section{Structured light for microscopy}

\section{Monika Ritsch-Marte}

Medical University of Innsbruck

\section{Status}

What we have

It seems amazing that light microscopy has remained such an active area of research to date, attracting thousands of researchers to large-scale optical microscopy conferences every year. Although breathtaking technological breakthroughs have been made, the major challenges are still the same [93]: (1) the poor contrast of unstained biological samples, (2) the limited spatiotemporal resolution, and (3) the difficulty of looking deep into a (scattering) sample with visible light. Structured light offers solutions addressing all of these challenges.

Concerning contrast, simple but effective methods have been developed to enhance contrast in thin unstained biological samples. Oblique illumination, schlieren and darkfield microscopy may be regarded as structured light methods tailoring or clipping rays in the illumination or the image formation pathways, respectively, to render transparent phase objects visible. Zernike phase contrast and differential interference contrast are more advanced, and there are widespread techniques capable of turning phase differences into intensity variations. In new developments programmable SLMs structure the light. Using such devices as programmable Fourier filters, one may emulate a chosen contrast technique in a phase sample 'on the fly' [84].

Alternatively, sophisticated and powerful staining methods have been developed. Fluorescence microscopy offers the opportunity for structural as well as functional imaging by labelling with fluorophores. It turned out that this route also brought a breakthrough in optical resolution, beyond the Abbe diffraction limit. This can be achieved by either shrinking the region where fluorophores can emit a signal during scanning using light structuring, as in STED, or by collecting information from sparsely emitting fluorophores, as in localization microscopy. Other examples of enhancing resolution by structuring the illumination and/or the detection are confocal laser scanning microscopy [94] and SIM [95], a wide-field method projecting grating patterns onto the sample.

The third challenge involves optical imaging or optical stimulation into the depths of a biological sample, e.g. for observing and stimulating living neurons in optogenetics [96]. Selective plane imaging uses a narrow light sheet for excitation of fluorophores inside a sample [97]. However, even in nonabsorbing, but strongly scattering, tissue the imaging depth is only a few hundred $\mu \mathrm{m}$. One way of solving this problem is to apply clearing agents [98], which seems incompatible with life cell imaging. Thus, recently, efforts have been made to profit from today's technologies for structuring light specifically for compensating tissue-scattering in order to focus deeper into the sample with visible light [99].
Structured Illumination Microscopy (SIM)

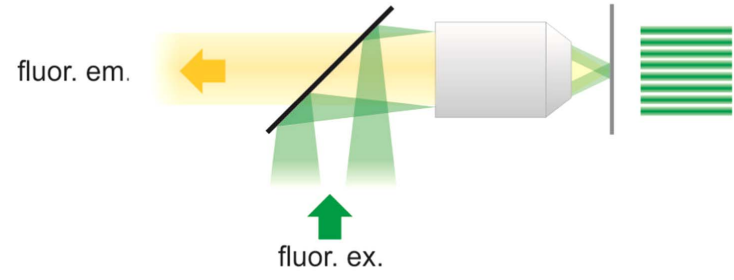

Stimulated Emission Depletion (STED)

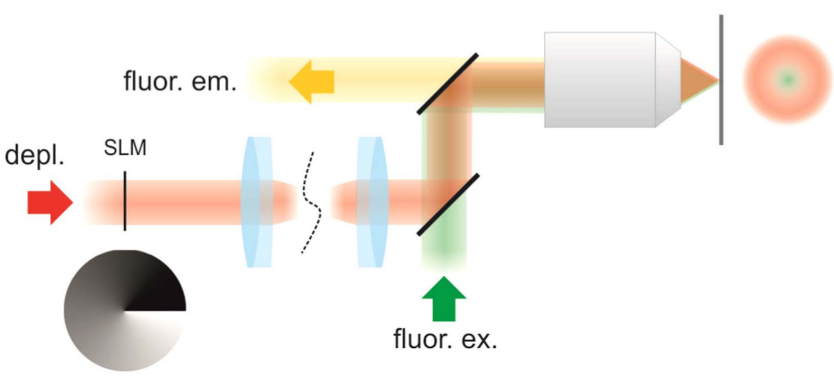

Figure 13. Examples of microscopy methods structuring light on the illumination side: structured illumination microscopy (SIM), STED.

\section{Phase contrast (PC)}

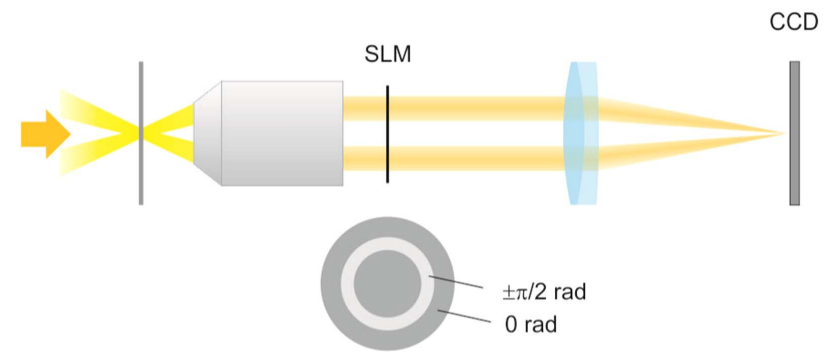

\section{Multiplane imaging}

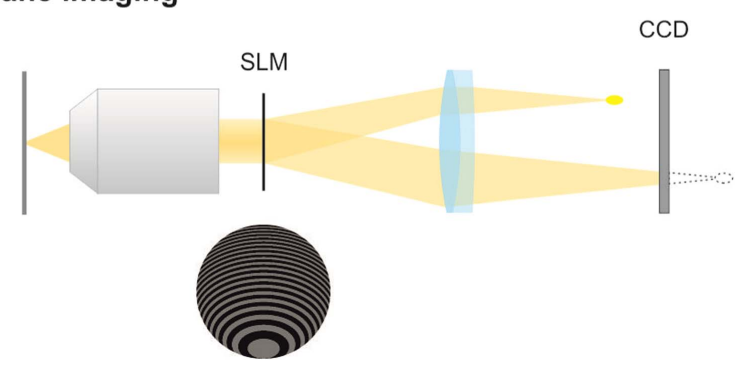

Figure 14. Examples of microscopy methods structuring light on the detection side: Fourier filtering, multiplexing.

\section{Current and future challenges}

\section{What we want}

Recent years have seen great advancements in optical microscopy, culminating in the Nobel Prize for Chemistry in 2014 for super-resolution fluorescence microscopy [100]. Nevertheless, there are still important issues concerning contrast, resolution, or accessible range, which currently 
hinders the progress of biological research, where structured light offers solutions [101].

Super-resolution mostly comes at the price of labelling the sample with fluorescent dyes, which are often unstable, phototoxic and change the sample in some way or other. In some instances, for example in STED microscopy operating at the highest resolution, high peak intensities are incident on the sample, and bleaching and photodamage become limiting factors. Structured light approaches are currently being investigated for their potential to optimally send photons to regions where they are needed and/or to keep them from areas where their presence is detrimental.

No method can provide all answers, as there will always be inevitable trade-offs. Therefore major efforts towards multi-modal imaging are being made. In particular, combinations of high-resolution (or even super-resolution) structural imaging with functional imaging have high potential of leading to entirely new insights into cell biology. Live cell imaging could greatly profit from a combination of superresolution and functional/chemically selective imaging (e.g. linear or nonlinear Raman). Structuring the illumination light -as well as the light scattered by the sample-will play a crucial role in meeting these goals. Combinations with methods involving plasmonic enhancements or nanostructured surfaces may lead to new types of imaging tools of unprecedented scope [102].

Optical methods still have to go deeper, for example to study neuronal networks or stem cell differentiation beyond the first few cell layers. It has been demonstrated recently that SLMs can 'undo' the aberrations induced by the sample itself. This not only enables a deeper look into the tissue, but also allows one to stimulate deeper structures, e.g. by locally uncaging substances by focusing light on the chosen spot. It requires fast and very precise control of the optical wavefront. Acoustic guiding or combinations with photoacoustics may push the limits even further.

\section{Advances in science and technology to meet challenges}

What we need

Wavefront shapers and SLMs were not originally designed for microscopy but for use in projection systems. Only recently they have evolved towards the needs of this new field of research application. Yet there is still ample room for improvements towards these goals.

For applications in neural stimulation, or for tailoring structured illumination beams, liquid crystal SLMs need to become significantly faster. Of course there is an intrinsic trade-off involving achievable phase shift and response time, which are both related to the thickness of the liquid crystal layer. Liquid crystal SLMs also tend to suffer from significant crosstalk, which limits the accuracy of the displayed phase pattern. Crosstalk is also a severe limiting factor in most deformable mirror devices, which are more suitable for correcting smooth aberration than for displaying a diffractive element. DMDs or digital light processors are currently designed for binary operation, as their mirrors only have two possible states, 'on' and 'off', a fact which strongly limits their efficiency.

An important measure for assessing the quality of an optical imaging system is the space-bandwidth product [103]. It is given by the number of degrees of freedom necessary to fully characterize an optical signal or an optical system. This makes the number of the pixels in the SLM essential, especially for off-axis imaging with both high resolution and large field-of-view. Thus affordable spatial phase modulators with a larger number of independent pixels would greatly benefit this research field. Ideally this development should be accompanied by faster response time and faster data handling.

The quality and the practicality of structuring light in microscopy also strongly depend on the available light sources. Intense light sources of tunable spectral bandwidth as well as tunable spatial coherence would be beneficial. Finally, microscopy will surely profit from scientific complementary metal-oxide semiconductor (CMOS) cameras with faster readout and lower noise.

\section{Concluding remarks}

It is difficult to predict what the optical microscope of the future will be able to do. It may be anticipated that programmable light beam structuring will eventually lead to systems that automatically adapt to the sample, optimizing contrast and resolution according to local needs and over a large dynamic range. One can also imagine the implementation of built-in trade-off management in the microscope that is based on fast, precise and efficient structuring of light.

\section{Acknowledgments}

The author thanks A Jesacher and G Thalhammer for helpful suggestions. 


\section{Structured light on the microscale}

\section{Natalia M Litchinitser}

University at Buffalo, The State University of New York

\section{Status}

The evolution and synergy of the fields of singular optics and nanostructured materials is poised to open entirely new opportunities in fundamental science and applications, including imaging, increasing capacity of communication systems through mode-division multiplexing, micromanipulation, and building higher-dimensional quantum encryption systems. Many of these applications require ultracompact sources of structured light, or beams carrying OAM that can be integrated on a chip. Such integration requires the availability of ultra-compact, tunable, ultrafast, low-loss components that can be efficiently coupled to the rest of a network, be integrated such that all interconnected optical and electrical components are arranged on a single substrate, and be compatible with a CMOS fabrication process.

However, until recently the majority of approaches to generating OAM beams have been based on macroscopic bulky optical components such as spiral phase plates, cylindrical lens converters, q-plates, SLMs, or specialty fibres. Some of the first realizations of a microscopic diffractive optical element for the generation of an OAM were demonstrated about ten years ago (figures 15(a) and (b)) [104, 105]. Recent progress in the field of nanofabrication and engineered optical materials, including metamaterials and metasurfaces, resulted in the demonstration of a number of structured light converters based on various material platforms [104-109].

Metamaterials embody a group of engineered materials where desirable material properties are engineered through the structure using meta-atoms. They have received significant research interest in the past decade and have resulted in the realization of a number of unique material properties that are not available in naturally occurring materials [110]. In particular, a miniaturized astigmatic optical element based on a single biaxial strongly anisotropic hyperbolic metamaterial was proposed to convert Hermite-Gaussian beams into vortex beams carrying an OAM and vice versa. Hyperbolic materials, have attracted significant attention in recent years owing to their extremely anisotropic properties and all-angle negative refraction with low loss [110]. The hyperbolic metamaterial behaves as a metal when light is passing through it in one direction and like a dielectric in the perpendicular direction. Such a material can be realized using an array of silver nanowires in a dielectric matrix, as shown in figure 15(C) [108]. The advantages of the proposed approach over the existing bulk optics based techniques for OAM beam generation include compactness and, therefore, compatibility with ultra-compact opto-electronic circuits, potential reconfigurability and an increased tolerance to misalignment.

\section{Current and future challenges}

While metamaterials have been predicted and demonstrated to produce some unique optical properties and functionalities, including the possibility of structured light generation, practical
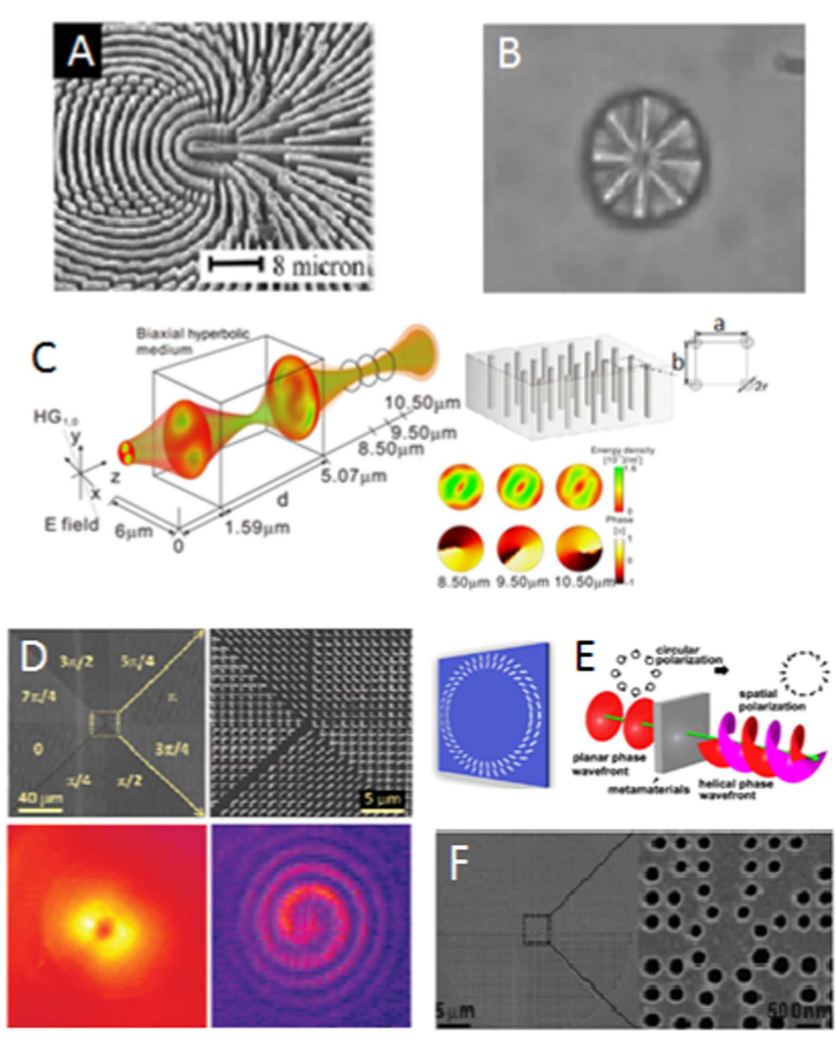

Figure 15. Examples of demonstrated microscale components for structured light generation. Panel (A), reprinted from [104], copyright 2005, with permission from Elsevier. Panel (B), from [105] copyright 2007 and panel (C, E), from [108] and [107] respectively, copyright 2013 reproduced with permission from The Optical Society. Panel (D), from [106], reprinted with permission from AAAS. Panel (F), reprinted with permission from [109], copyright 2014 American Chemical Society.

obstacles precluding their transition to practical applications and mass production are high losses, difficulties in fabricating 3D nanostructures, and incompatibilities of current material platforms ( $\mathrm{Ag}$ and $\mathrm{Au}$ ) with the existing CMOS process.

Some of these challenges can be overcome using so-called metasurfaces that are 2D artificial materials with thicknesses much smaller than the incident light wavelength, allowing complete control of the phase, amplitude, and polarization of light beams. Compared to conventional optical elements, which rely on long propagation distances, these devices facilitate strong light-matter interaction in sub-wavelength-width material layers, allowing abrupt changes of beam parameters.

Recently, various approaches and designs of metasurfaces have been demonstrated. An important class of such devices include space-variant Pancharatnam-Berry phase optical elements (PBOEs) [104]. The phase change in the PBOEs results from the geometrical phase that accompanies space-variant polarization manipulation, rather than arising from optical path differences as in conventional diffractive and refractive elements. A novel approach to beam manipulation using flat optics based on plasmonic antenna arrays was shown to enable novel flat lenses and optical components for creating optical vortices (figures 15(D) and (E)) [106, 107].

In another approach, a nanowaveguide array with gradually changing nanowaveguide radii milled in a metal film was proposed and demonstrated to control the wavefront of a light 
beam (figure $15(\mathrm{~F})$ ) [109]. The basic idea of such a device is as follows. Each nanowaveguide introduces a specific phase change determined by its radius. Therefore, a spatial distribution of the nanowaveguide radii can be optimized such that a total phase change of $2 \pi$ is imposed on the wavefront of the beam upon its propagation through such an array. As a result, a laser beam passing through the designed array would acquire an OAM and would be transformed into a vortex beam with a topological charge of one. Such a design is ultra-compact and can be readily integrated on a chip or on a transverse crosssection of an optical fibre.

\section{Advances in science and technology to meet challenges}

While metal-dielectric metasurfaces, being 2D structures, partially solve the fabrication challenges associated with the realization of metamaterials-based structured light components, they still have a relatively low efficiency due to orthogonal polarization coupling and ohmic losses in metals. Moreover, they may not be compatible with existing CMOS processes.

A promising alternative solution relies on all-dielectric resonant structures. In one approach, ultra-compact siliconintegrated optical vortex emitters, which rely on whispering gallery modes of a circular resonator, were demonstrated to emit a beam with well-controlled amounts of OAM into free space (figure 16(A)) [111]. Very recently, a microring laser producing single-mode OAM vortex lasing with the ability to precisely define the topological charge of the OAM mode was reported (figure 16(B)) [112].

Another approach is based on the possibility of exciting both the magnetic and electric resonant responses of highrefractive index dielectric nanoparticles in the same frequency range. The presence of both electric and magnetic resonances at the same frequencies allows full $2 \pi$ phase control in a singlelayer all-dielectric structure necessary for structured light generation (figures 16(B) and (C)) [113, 114]. In particular, it was shown that silicon nanostructures, having a relatively highrefractive index at telecommunication wavelength, can be optimized to possess overlapping electric and magnetic dipole resonances in the same frequency range. Such all-dielectric nanostructures enable high transmission efficiency since they do not suffer from intrinsic nonradiative losses in metals. Additionally, dielectric metasurfaces, unlike their 3D analogues, metamaterials, do not require complicated fabrication techniques and can be produced in one lithographical step.

Future advances necessary to transform this all-dielectric based approach from laboratory experiments to practical design and manufacturing requires further developments in numerical modelling techniques for efficient design and optimization of these structures, as well as integration of these devices on a chip and/or with optical fibre technology.

\section{Concluding remarks}

In summary, engineered nanostructures are poised to empower the science and applications of structured light on the microand nanoscales, including trapping and optomechanical micromanipulation, optical microscopy, advanced communications,
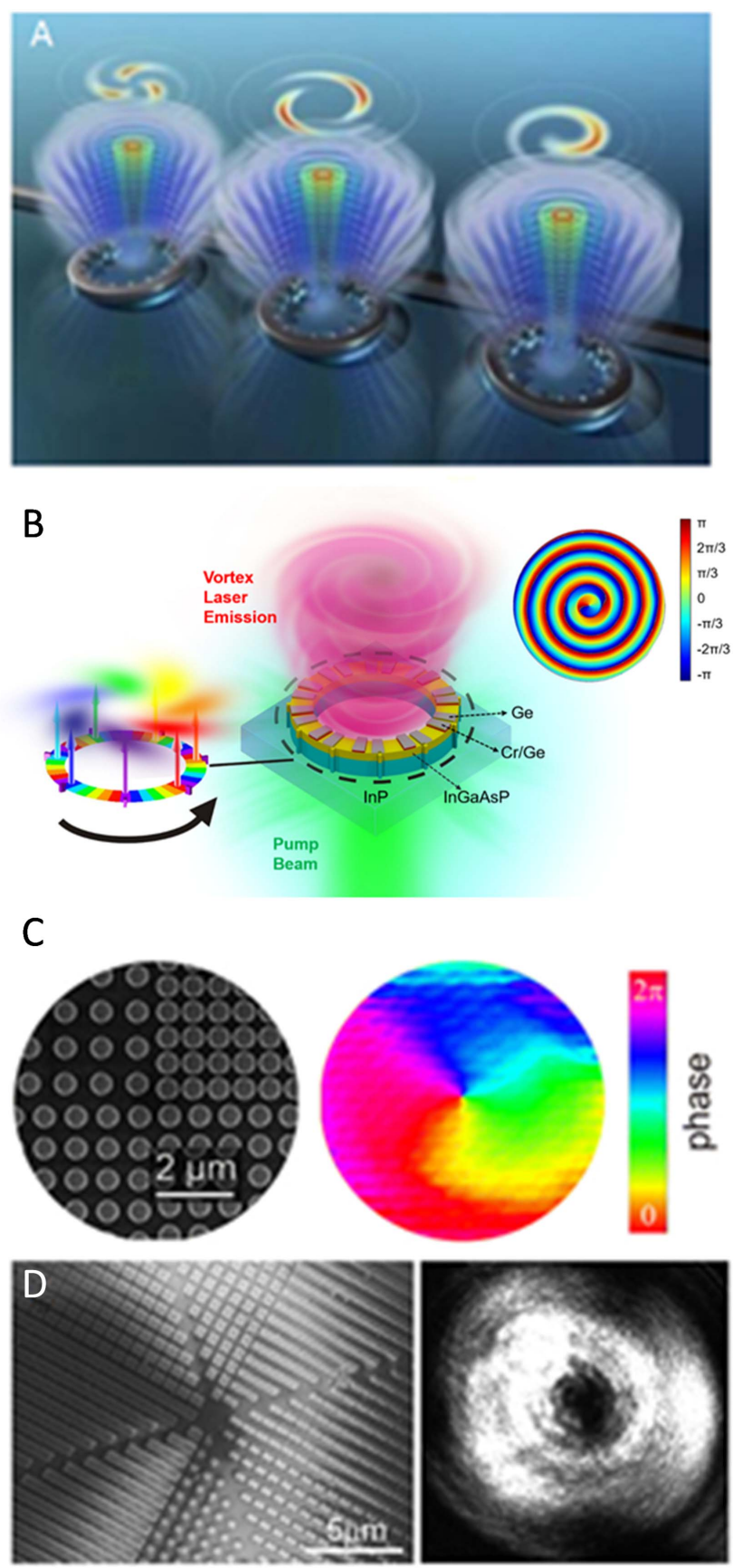

Figure 16. Vortex emitters and dielectric metasurface-based components for vortex generation. Panel (A), from [111], reprinted with permission from AAAS. Panels (B, C) and (D) reprinted with permission from [113] and [114], respectively, copyright 2015 American Chemical Society.

and quantum information. Recent developments in the field of silicon-based OAM components offer a practical route to the realization of ultra-compact, efficient devices compatible with current semiconductor technologies.

\section{Acknowledgments}

We would like to acknowledge the support of our work on Structured light-matter Interactions Enabled by Novel Photonic Materials by the Department of Energy Award DE-SC0014485. 


\section{Application of structured light to ultracold atoms: controlling the motion and quantum state, and creating singular optic analogues}

\author{
Nicholas P Bigelow \\ University of Rochester
}

\section{Status}

Due to their highly controllable and spatially dependent amplitude, phase, polarization and angular momenta, structured light beams offer new opportunities to advance work with cold atoms. In this section we consider three possibilities: (1) the control of atomic motion, (2) the manipulation of the quantum state of a quantum degenerate gas and (3) the emulation of singular optical states using matter waves.

A classic example of the control of atomic motion is optical trapping. By taking advantage of a dark central intensity profile that can be created in structured laser beams, several groups have investigated novel optical dipole trapping schemes in which the atoms are contained in a dark zone by a repulsive wall of light [11]. In other examples, laser light that was structured holographically or using conical refraction was used to implement novel atom traps [115]. These traps have the advantage of minimizing photon scattering and parasitic decoherence and heating effects. A related application of structured light to cold atoms is atomtronics. For example, researchers have recently created a toroidal optical dipole trap able to contain a superfluid Bose-Einstein condensate. By introducing a weak radial barrier at one point in the torus, an atomtronic circuit element with a tunable weak link was realized [116] thereby opening the door to the realization of an atomic SQUID analogue.

A close relative of atomtronics is atom optics, which is the manipulation of atomic de Broglie waves (matter waves) in analogy to the manipulation of light waves in optics [117]. Experiments have demonstrated mirrors, lenses, beam splitters, phase gratings and ultra-sensitive atom interferometers. In atom optics the underlying physical processes are considered coherent in that the atom optical element depends on affecting the phase evolution of the individual incident atomic wavepackets. When the atoms are part of a Bose-Einstein condensate (BEC) the meaning of coherent is extended to refer to the manipulation of the entire condensate wavefunction (amplitude and phase) coherently. BEC-based atom optics and atomtronics have enabled quantum and nonlinear atom optics experiments, including four-wave mixing, atom lasers, atom transistor analogues, and more. A future direction is to ask (1) are there additional ways in which a particular form of structured light, for example singular optical fields $[2,3,118,127]$, can be used to manipulate the quantum state of a degenerate (BEC) atomic sample? (2) Are there atom optical equivalents to singular optics that open the door for 'singular atom optics'?

As noted, structured light has been used to investigate novel cold atom traps. In $[119,120]$ the interaction of singular light with ultracold atoms instead exploited the fact that Laguerre-Gaussian laser beams can transport OAM. In [120]
OAM-carrying beams were used to drive coherent Raman transitions between different Zeeman sublevels of the ground state atoms in a spinor BEC. During the Raman transition, conservation of spin and OAM, together with the atom-field dynamics, enables the singular optical fields to engineer the spinor BEC wavefunction. The light in the Raman beams affects the spatial distribution of the individual spin states in the BEC as well as their relative phases. The position-dependent phase of each of the spin components is also sculpted by the singular optical fields. As a specific example of this 'phase imprinting' using an OAM beam, a $2 \pi$ azimuthal phase winding can be created in the BEC [119]. The azimuthally varying phase twist represents a superfluid vortex that carries OAM through atomic motion. Matter wave interference reveals the azimuthal phase of the BEC. The spiral pattern in figure 17 represents a superposition of counter rotating interfered vortices in the BEC; it matches the pattern that would be obtained in a purely optical interference experiment involving Gaussian and Laguerre-Gaussian beams.

Singular optical fields can also be used to create topologically singular states in a BEC such as spin monopoles, skyrmions, Alice vortex states, and more [120]. This demonstrates that the combination of singular optical fields and BEC physics opens the door not only to singular atom optics, but also for new forms of quantum simulation, BEC and Hamiltonian engineering, and for new approaches to the investigation of synthetic gauge fields [121].

Aspects of structured light can be explored by combining singular optics with atom optics. In singular optics an interesting family of optical states involve those that contain polarization C-point singularities. These states have names such as star, lemon and monstar [122]. There is an analogy between atomic pseudo-spin (described on the Bloch sphere) and optical polarization (described on the Poincaré sphere) that can be exploited. This analogy enables the realization of the atom optical versions of birefringent optical components such as waveplates and q-plates [123]. In turn these atom optical elements enable the creation of atomic analogues of the optical C-point singularities in the atomic spin texture.

\section{Current and future challenges}

One important aspect of singular atom optics is that, unlike singular optics with photons, atoms can be nearly stationary and can interact with each other. These interactions have many consequences including affecting the symmetry group relevant to describing the quantum state of system-a rich and complex degree of freedom inaccessible in photonic optics. Furthermore, while optical polarization is cast as a two-state manifold (left-right circular basis or perpendicular linear bases), atomic spin can offer a much larger and richer state space [124]. Thus, the use of atomic de Broglie waves to simulate singular optical states will allow the emulation of higher-dimensional Poincaré states of optical polarization and optical angular momentum which have, to date, only been considered theoretically [124]. Understanding the properties of these complex state spaces is fundamental to both the 


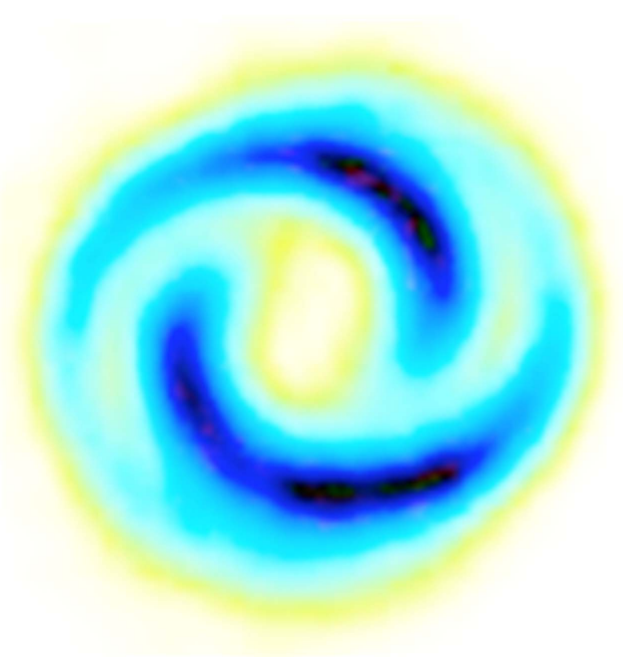

Figure 17. The density profile of one spin state of a spinor BEC that has counter rotating azimuthal phase twists (counter rotating vortices) and a radial phase gradient. See [119].

physics of complex light and the optical creation of topological structures of a BEC.

Singular optical beams are also of interest for applications in classical and quantum communication and for information processing. For example, investigations are underway to determine whether Laguerre-Gaussian beams are intrinsically less susceptible to distortion when propagation than simple Gaussian beams. In addition, entangled photons that carry OAM have been created. In almost any vision using singular optics in information processing, the need for a memory is obvious. Ultracold atomic gasses are excellent candidates for the memory. In an elegant experiment in Paris [125] a quantum memory for OAM qubits was demonstrated in a cold atom sample. However, the development of the approach as a robust quantum technology remains a challenge, and the use of a BEC should offer significant enhancements in storage time.

\section{Advances in science and technology to meet challenges}

Much of the work described in this chapter is technology enabled and a number of these technologies are described elsewhere in this roadmap. Consider one possible application specific to applying structured light to work with ultracold atoms. For both quantum simulation and quantum information physics, creating arrays of micron-sized sculpted atom optical states in two and three dimensions is a frontier.
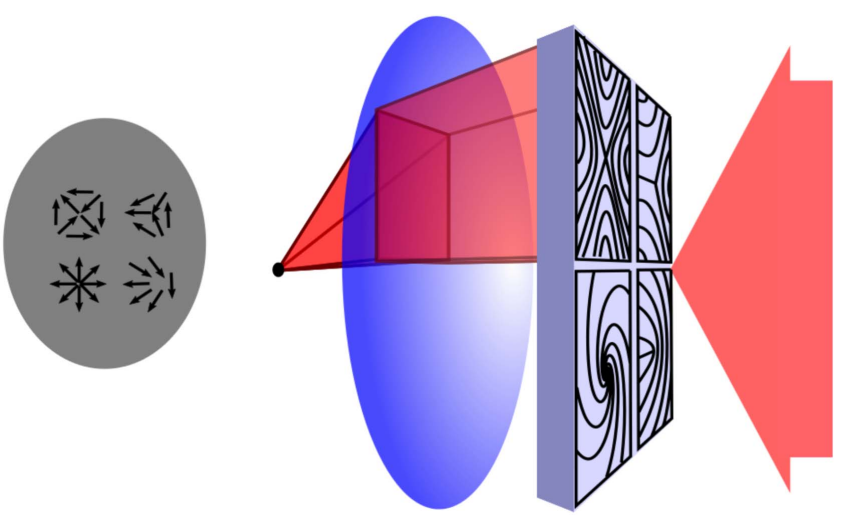

Figure 18. Arrays of individually sculpted spinor BEC structures can be engineered by creating structured light using an SLM array, here shown imprinting four singular beam regions, and the imaging the beams through a microscope objective onto the condensate.

Technical advances such as SLMs and q-plates enable highly complex singular and non-singular optical fields to be created for next generation BEC quantum state sculpting. Creating microscopic arrays requires new approaches. As shown in figure 18 , one can, for example, envision a modification of the recently developed quantum gas microscope [126] involving structured light input fields. In the atom microscope a high performance microscope objective is placed only millimetres away from a BEC, separated by a thin window of a glass vacuum cell. By creating a structured light array as the input to the microscope it may soon be possible to write complex structures in an atomic sample and to study their evolution and interaction.

\section{Concluding remarks}

The application of structured light to atom optics and to research on cold quantum gases has enabled a wide range of experimental opportunities. We see an exciting future for experimental and theoretical progress for both fundamental studies and next generation applications.

\section{Acknowledgments}

This contribution to the roadmap is the work of the University of Rochester Cooling and Trapping group and specifically, Azure Hansen and Justin T Schultz, with help from Joseph D Murphree and Maitreyi Jayaseelan. The group is supported by the NSF and NASA. 


\section{Structured light for metrology}

\author{
C Rosales-Guzmán ${ }^{1,3,4}$ A Belmonte ${ }^{2}$ and J P Torres ${ }^{2,3}$ \\ ${ }^{1}$ University of the Witwatersrand \\ ${ }^{2}$ Universitat Politecnica de Catalunya \\ ${ }^{3}$ ICFO-Institut de Ciencies Fotoniques, The Barcelona Insti- \\ tute of Technology \\ ${ }^{4}$ Author to whom any correspondence should be addressed.
}

\section{Status}

The ability to generate light fields with almost any desired transverse phase structure has renewed interest in generating and analysing optical fields with new shapes, with the goal of finding new solutions to old problems. Structured light has proved to be a very useful tool in fields as diverse as optical communications, super-resolution microscopy, optical tweezers and quantum optics to mention just a few [127]. For instance, the real-time manipulation of structured light allows for the study of the interaction dynamics of phase singularities with other systems [128], as well as the generation of beams with exotic properties such as self-healing [129].

Novel techniques involving the manipulation and structuring of the optical phase have been recently introduced in the field of optical metrology. In laser remote sensing, a technique for measuring directly the transverse component of the velocity of a target has been proposed. The phase of the illumination beam is structured in a suitable way according to the target's motion to be detected (see figure 19(a)). In this way, the light scattered back from the target contains information about its position and velocity at each instant. The information is extracted by interfering it with a reference field $[130,131]$. The principle behind this technique has reached the field of fluid dynamics where it has been demonstrated to be capable of measuring directly the local vorticity of a flow [132]. In the field of optical activity, a novel approach to linking the interactions of chiral molecules with structured light has recently been introduced. It is known that chiral molecules distinguish left-handed from right-handed polarized light by absorbing them at different rates, a phenomenon known as circular dichroism. In this new approach, the handedness of twisted light, that is, structured light with an azimuthally varying phase, has been demonstrated to produce an enhanced interaction with chiral molecules [133]. In the field of profilometry, a technique based on mode projection, was introduced to overcome some of the major drawbacks of current techniques for thin film measurement. Highly sensitive interferometric techniques used to accurately measure small thicknesses should often operate at the quadrature condition. For this, it is necessary to build a wavelength dependent ridge to place the sample. In the mode projection approach, the light reflected from the sample is projected onto an appropriately tailored mode in which the quadrature condition is imposed, independently of the wavelength [134].
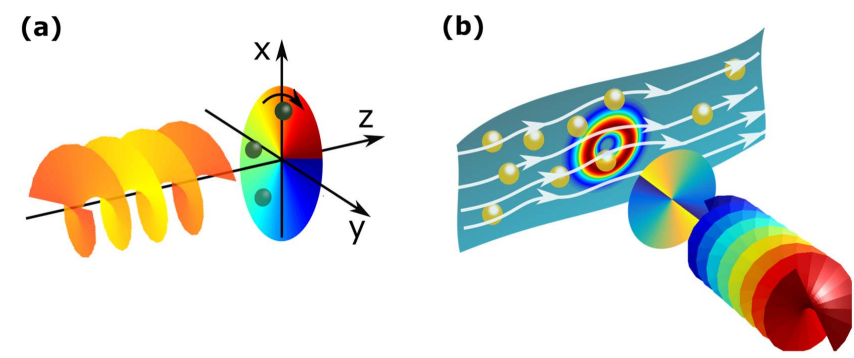

(c)

(d)
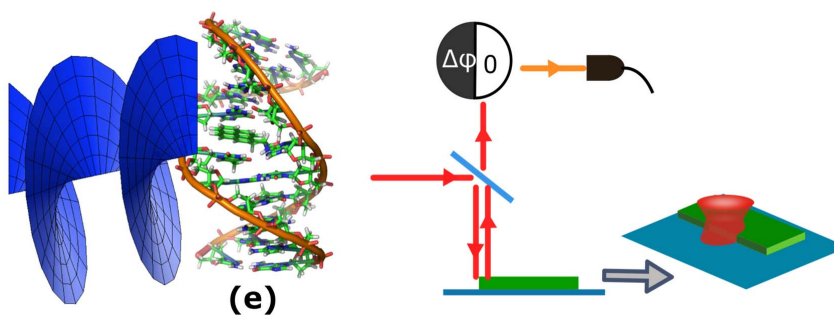

Figure 19. Applications of structured light to: (a) laser remote sensing, (b) vorticity in fluids, (c) optical activity in chiral molecules and (e) thin film measurement.

\section{Current and future challenges}

Structured light has proved to be a powerful tool in a wide variety of fields. However, its successful deployment in outof-the-lab environments still depends on the further development of certain crucial technologies in order to produce the appropriate devices to generate structured fields in such conditions and to merge these with the required application.

On the generation side, current technology provides powerful devices to reshape Gaussian beams into structured fields, such as SLMs, one of the most versatile devices available in the market. SLMs are high-resolution liquid crystal devices capable of generating a myriad of structured fields. A computer-generated hologram, designed to produce the required field, is displayed on a liquid crystal device. The flat phase of a Gaussian beam is then transformed into a structured field. The refresh rate of SLMs can be as high as high as $60 \mathrm{~Hz}$, allowing for the rapid generation and switching of structured fields. Some of the disadvantages of present SLMs are their market prices, and in some cases their volume, making its incorporation into certain applications difficult. Hence, one of the biggest challenges of current technology is perhaps the mass production of cheaper and smaller devices customized for each application.

On the application side, the use of structured light for metrology unveiled other technological challenges. The biggest is perhaps the incorporation of structured fields into existing devices to improve their capabilities. In the case, for example, of laser remote sensing, current systems can measure velocity, and its magnitude and direction, making use of heterodyne detection. For its implementation, an optical frequency offset between the illumination and the reference beam is introduced. This offset can be achieved by mechanical means, for example using rotating waveplates, or through 
the use of acousto-optic modulators and electro-optic frequency shifters. A drawback of these systems is the need for customization to a particular beam size and specific frequency. The incorporation of dynamic structured fields into current systems can provide alternative methods that do not require the use of additional optical elements. One way to implement this technique is through the use of SLMs, but its low refresh rate represents a major barrier [135].

In some cases, the usefulness of using structured light has been demonstrated only theoretically. Therefore one of the first challenges is its experimental demonstration. For example, even though it has been predicted an enhanced response of chiral molecules, upon illumination with azimuthally structured light, this prediction has not been observed yet.

\section{Advances in science and technology to meet challenges}

Even though SLMs have noticeably advanced the generation of structured light fields, their price represents a major disadvantage. On top of that, their low refresh rate, polarization and wavelength dependency and efficiency sometimes might become an issue. The DMD offers a promising alternative. A DMD is a chip composed of several hundred thousand microscopic mirrors arranged in a rectangular array (figure 20(a)). Each mirror can be controlled individually by rotating it $\pm 10^{\circ}-12^{\circ}$, to an 'on' or 'off' state. DMDs are orders of magnitude cheaper, polarization insensitive and their refresh rate can reach a few $\mathrm{kHz}$. Even though DMDs have been used to generate structured fields, their modulation efficiency is still a challenge.

The combination of both technologies, SLMs and DMDs, would bring significant advances to transfer novel ideas from a laboratory concept to a real application. But technology also has to advance in other directions to incorporate new ideas into existing devices. For example, in the case of laser remote sensing, the technology for monitoring moving targets is well known. Current systems employ Gaussian laser beams but certainly the addition of structured fields would significantly expand their capabilities. One can envision a laser system that measures at once both the longitudinal and the transverse components of velocity. This possibility has been conceptually demonstrated in the laboratory, where the alternation between two different fields, Gaussian and azimuthally structured, allows the ful1 characterization of 3D helical motion [136]. Here, an SLM allows changing from one field to the other, inherently limiting this technique to the refresh rate of the SLM.

\section{Concluding remarks}

In summary, structured light fields are increasingly finding applications in a wide variety of fields. They have shown their (a)
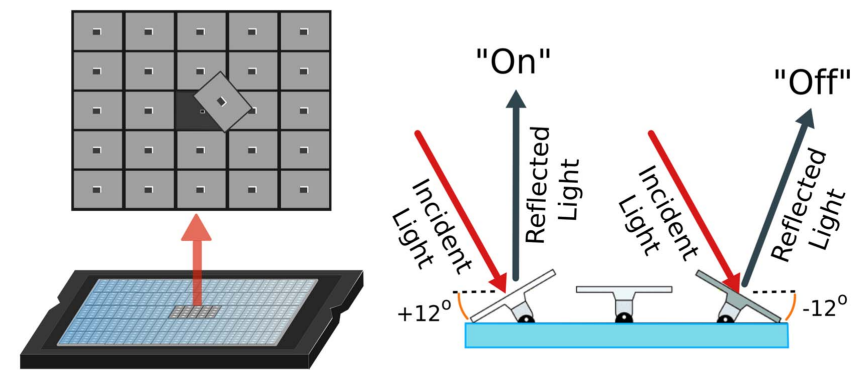

(b)

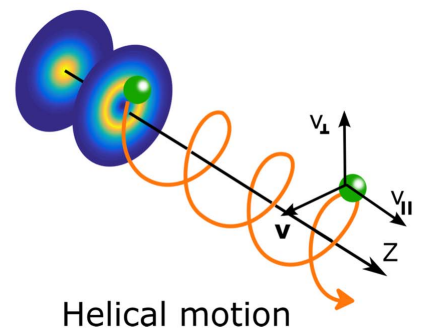

Figure 20. (a) The DMD can be used to generate structured fields Each mirror can be set to an 'on' or 'off' state by rotating it from $+12^{\circ}$ to $-12^{\circ}$. (b) Full characterization of $3 \mathrm{D}$ motion through the use of structured light.

potential where commonly used Gaussian beams have encountered physical limitations. Up to now, applications have been discovered in fields as diverse as optical communications, optical manipulation, super-resolution microscopy, and optical metrology but many more are yet to be found.

Moreover, one of the most versatile devices for generating structured light fields is the SLM, but it is a technology not affordable by everyone and bears some other disadvantages. A potential alternative lies in the technology of the DMD that, although it has some issues of its own, is at least an order of magnitude cheaper than equivalent SLM devices.

Indeed, one of the major challenges for future applications is the production of cheaper, faster and highly efficient devices for generating structured fields. This will significantly impact on other fields of research by them benefiting from the use of structured fields.

\section{Acknowledgments}

CRG would like to acknowledge support from CONACYT. 


\section{Structured light for atomtronic devices}

Tyler W Neely, Mark Baker, Halina Rubinsztein-Dunlop

University of Queensland

\section{Status}

Atomtronics is an emerging field of application for quantum gases that seeks to implement analogues of electronics circuits in superfluid systems [137]. As such, the application of analogues to circuit elements (capacitors/inductors/resistors [138], transistors/diodes/batteries [139, 146]) has recently been demonstrated. These lumped elements rely on the precise control of both the confining potential, where superfluid flow is analogous to electric current, while extrinsic properties such as the system chemical potential form is analogous to the voltage applied across a conventional circuit.

Confining potentials for quantum gases generally may be either magnetic or utilize the optical dipole force resulting from a far-off-resonant optical beam. As the ability to microscopically configure magnetic potentials is spatially limited by the macroscopic current-carrying wires, structured light has emerged as the tool of choice for the production of configurable potentials at the micron-scale.

The earliest examples of atomtronics with bosonic and fermionic quantum gases were a series of impressive experiments in optical lattice potentials. Utilizing the standing wave interference of multiple optical beams to produce an optical lattice, these systems represent a remarkably simple implementation of structured light. Lattice systems are well described by the Bose-Hubbard or Fermi-Hubbard Hamiltonian, and are in close analogy to electrons in a periodic lattice, and exhibit a band structure and insulating states. Even for the bosonic case, the typically repulsive mean field interactions lead to a decreased likelihood of finding two bosonic atoms together in the same lattice site, in close analogy to solid state electronics [137]. Lattice methods have enabled the recent demonstration of diode-like negative differential conductivity in an atomtronic device [139].

The extensive research on the use of structured light for optical tweezers has also given atomtronics a suitable starting point for the production of more complex potential landscapes. These techniques, as applied to quantum gases, can be generally categorized into two classifications-those based on time-averaging methods and those utilizing SLMs in either a Fourier or direct imaging plane. We review first the progress that has been made with time-averaged methods.

The most configurable time-averaged traps have been produced from the rapid angular modulation of a Gaussian beam with a two-axis acousto-optic modulator. As the Bosecondensed atoms in a typical quantum gas experiment approach temperatures in the $\mathrm{nK}$ regime, for suitably weak confinement, the atoms can only follow the time average of the scanned beam. This means that raster scanning can be used to rapidly trace complex planar patterns, while vertical confinement of the atoms is provided through an attractive Gaussian sheet, as shown in figure 21. First introduced in the

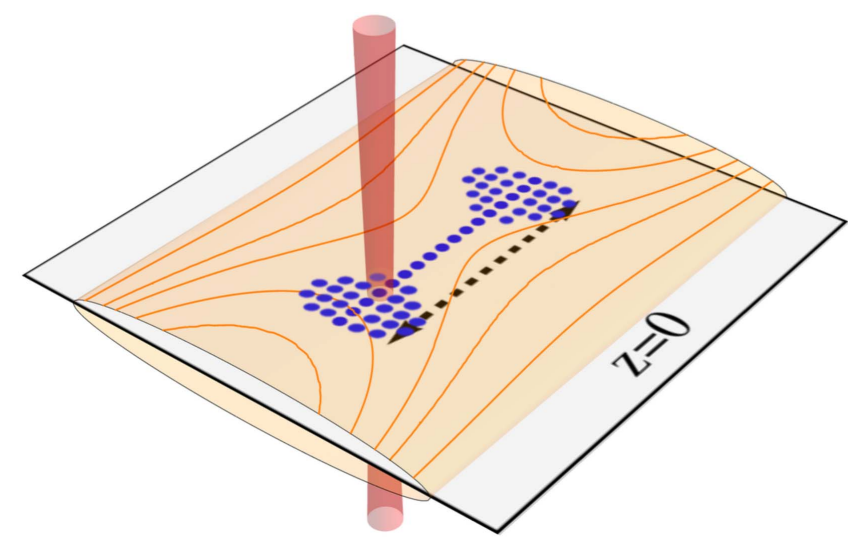

Figure 21. The time average technique for creating a dumbbell potential, an atomtronic analogue of an RLC circuit. By rapidly scanning the focused beam between the points indicated, a timeaveraged potential is generated.

context of ring traps for Sagnac effect-based interferometers, these methods have since been expanded to producing atom waveguides [140], large ring traps, and extended line traps [141]. As the ultracold atoms are confined to the absolute minima of the final potential, smoothness of the scanned pattern is of paramount importance, and recently feed-forward methods have been used to correct scanned potentials, resulting in a large degree of uniformity [141]. These methods have been used to produce a barrier across a ring-shaped trap to realize a weak-link atomtronic Josephson junction [142].

The production of static and/or quasi-static structured potentials for atomtronic applications means SLMs have been implemented in quantum gas experiments. In optical tweezer applications SLMs are most often used in the Fourier plane. In this implementation, the SLM controls the phase and/or amplitude of an input Gaussian optical beam, with the pattern representing the spatial Fourier transform of the desired amplitude pattern. The optical system then focuses this sculpted light pattern on the plane containing the atoms, performing a Fourier transform and recovering the desired spatial pattern. These methods can, however, suffer from laser speckle (optical vortices) resulting from both digitization errors and also defects in the phase pattern from algorithmic optimization of the SLM pattern. As cold atoms are sensitive to defects in the trapping potentials at the $\sim 1 \%$ level, optical speckle has limited the application of phase-based Fourier plane SLMs in quantum gases [143]. Recent progress on optimizing these patterns has, however, been promising, and it is expected that successful implementation of arbitrarily configured trapped atoms using Fourier plane methods will be demonstrated soon. However, Fourier plane methods are particularly useful for phase imprinting, as has been demonstrated successfully to imprint vortex/skyrmion structures [144].

A simpler and, to date, more successful methodology for creating configurable optical potentials for quantum gases is the direct imaging of amplitude patterns. This may be as simple as the use of a fixed mask, which has been utilized to 
produce double-well dumbbell potentials (analogous to an RLC circuit) [138]. Though amplitude based direct imaging methods are less commonly encountered in optical tweezers, the method of generalized phase contrast utilizes interference between a spatially configured phase pattern and reference beam to produce an intermediate amplitude pattern, subsequently reimaged to the trapping plane. This method has been utilized to realize a double-well potential $\mathrm{RC}$ atomtronic circuit [138].

A recently emerging and powerful amplitude based method is the use of DMDs for configuring the amplitude of an input beam either in the Fourier plane or in a direct imaging configuration [145]. In the latter case, the implementation is similar to the fixed mask technique mentioned above, but with the advantage of being dynamically configurable. The rapid switching capability of DMD devices $(\sim 20 \times$ that of a comparable phase-modulating SLM), and the ability to store large numbers of frames holds great promise for high precision and dynamic atomtronic potentials for quantum gases derived from DMDs.

\section{Current and future challenges}

We summarize here the trade-offs between the different techniques shown in figure 22. Time-averaged traps may still introduce density modulations to the trapped gas, even at scan frequencies above $100 \times$ the relevant trapping frequency. Such methods are however advantageous for easily producing large configured trapping patterns.

SLMs in the Fourier plane have been largely limited by laser speckle, but with algorithmic optimization of the patterns improving, the shortcomings and drawbacks of using them are to large extent gone. Such methods can be quite light efficient, with diffraction efficiencies above $50 \%$. However, producing large high-resolution patterns remains challenging as accuracy suffers at the high diffraction orders required.

Direct imaging methods, especially those utilizing DMDs, appear well suited for the generation of high-resolution patterns of larger spatial extent when compared with phase-based methods. This however comes at the cost of light efficiency, as the light illuminating mirrors in the 'off' state are lost from the optical system.

Full control of both the amplitude and phase of quantum matter waves appears possible with a combination of these various technologies. However, the requirements for this

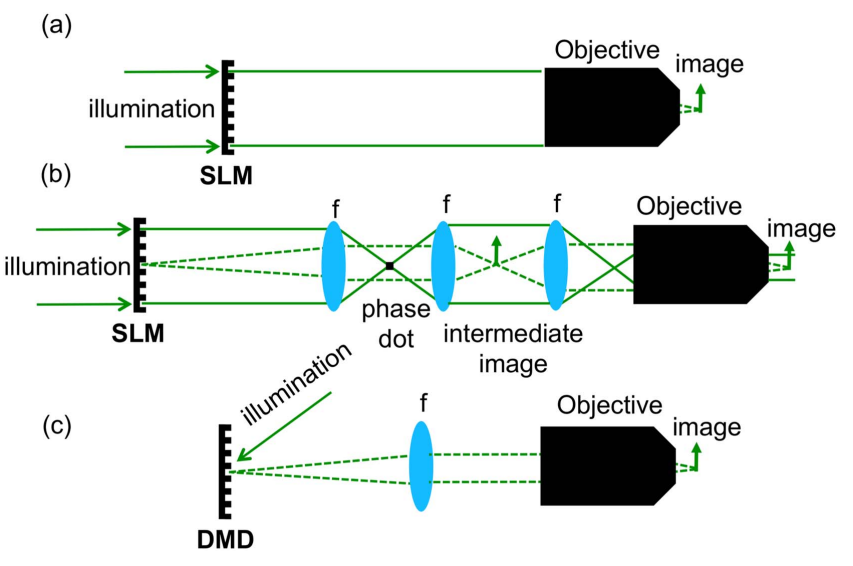

Figure 22. Comparison of the three SLM methods: (a) a phase or amplitude modulated SLM is illuminated with a collimated beam, resulting in the desired Fourier transform at the image plane; (b) interference with a phase dot results in an intermediate image that is reimaged; (c) direct imaging of a DMD.

remain technically challenging, as quantum gases have only just begun to be arbitrarily configured at their smallest (submicron) length scales, as required for the implementation of tunnelling-based devices such as atomtronic diodes [139].

\section{Concluding remarks}

Atomtronics seeks to implement analogues of electronic devices using ultracold quantum gases. Structured light provides tools enabling this field, complimenting the work done using atom chips [146]. Such optical methods benefit from the rapid improvements in the precision and flexibility of optical trapping techniques, and full control of both the amplitude and phase of quantum gases in configured geometries appears feasible in the near future.

\section{Acknowledgments}

The Bose-Einstein condensation group at the University of Queensland is supported by the ARC Centre of Excellence for Engineered Quantum Systems (CE1101013), and ARC Discovery Projects (DP160102085). 


\section{Nanoaperture tweezers: structured light at the nanoscale}

\author{
Reuven Gordon \\ University of Victoria
}

\section{Status}

Light-matter interactions can be enhanced by structured light at the nanoscale. This is of particular benefit for light interactions with single nanoparticles, such as colloidal quantum dots, upconverters, viruses, DNA fragments and proteins. To achieve structured light at the nanoscale, many researchers have used nanoplasmonics, shaping metals at the nanoscale to control the flow of light.

Here, I focus on the application of structured light to nanoaperture optical trapping tweezers, or nanoaperture tweezers. In 2009 it was shown that a single circular aperture in a metal film could achieve stable trapping of $100 \mathrm{~nm}$ diameter polystyrene beads while using less than a milliwatt of laser power [147]. That was considered a significant step forward because for the first time it was possible to overcome the limitations of the strong size-dependence of Rayleigh scattering and thereby to hold nanoparticles with low optical powers. The trick was to use the sensitivity of Bethe apertures to dielectric loading in order to overcome the limitation of Rayleigh scattering. As an added benefit, the trapping was easily monitored by just observing large changes in optical transmission through the aperture.

Following that work, we recognized that shaped apertures (beyond the simple circle) could be used to trap smaller nanoparticles, single proteins [148], and single-stranded (ss) DNA. The shaping was required to structure the optical field at the nanoscale level to be comparable in dimension to the nanoparticle being trapped. Figure 23 shows experimental data of a $20 \mathrm{~nm}$ polystyrene sphere trapping with a doublenanohole aperture shape. With shaped apertures, it has been demonstrated that nanoparticles could be trapped and translated at the end of a near-field tapered fibre optic probe [149], opening up possibilities in nanoassembly and particle selection/isolation.

This work demonstrated the potential of using aperture tweezers for single molecule biophysics and nanotechnology. Using this platform in conjunction with established practices in lab-on-chip, spectroscopy and microscopy (including scanning probe), it is anticipated that powerful new approaches will emerge. For example, by interfering two trapping laser beams of slightly different frequency, we were able to 'listen' to the acoustic vibrations of nanoparticles in an approach termed extraordinary acoustic Raman (EAR) spectroscopy [150]. EAR spectroscopy has been applied to colloids, proteins [150] and ssDNA (see figure 24). This technique makes use of temporal structuring of light to modulate the electrostriction force at frequencies from the $\mathrm{GHz}$ to the $\mathrm{THz}$ range.

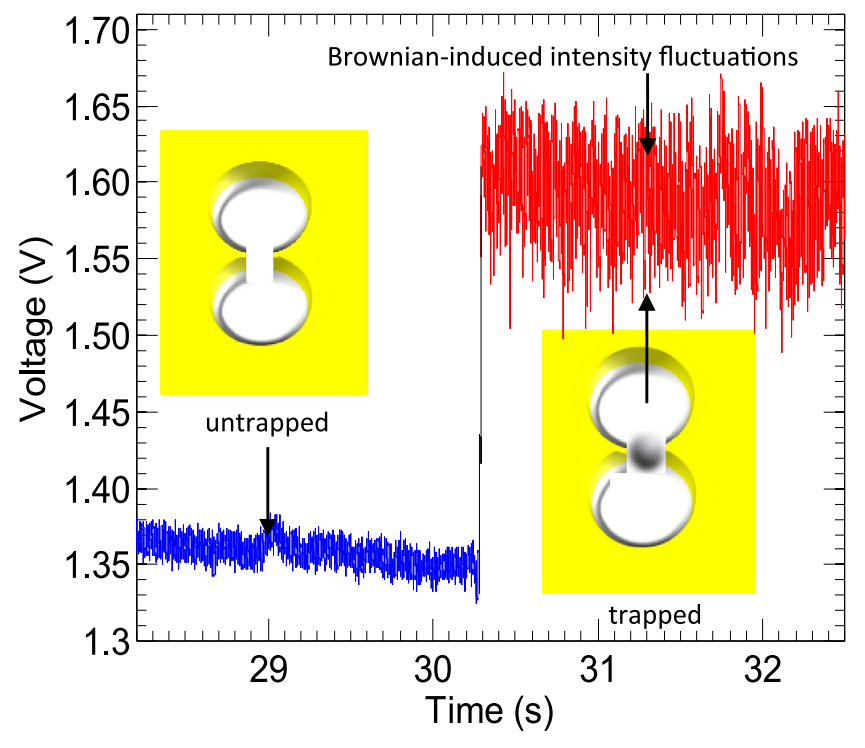

Figure 23. Change in laser transmission through a double nanohole in $100 \mathrm{~nm}$ thick gold film with trapping of a $20 \mathrm{~nm}$ diameter polystyrene sphere (nanoparticle) in solution. The transmission is proportional to the avalanche photodetector voltage. An abrupt step occurs at trapping (20\% jump in transmission), as well as an increase in intensity fluctuations from Brownian motion of the nanoparticle.

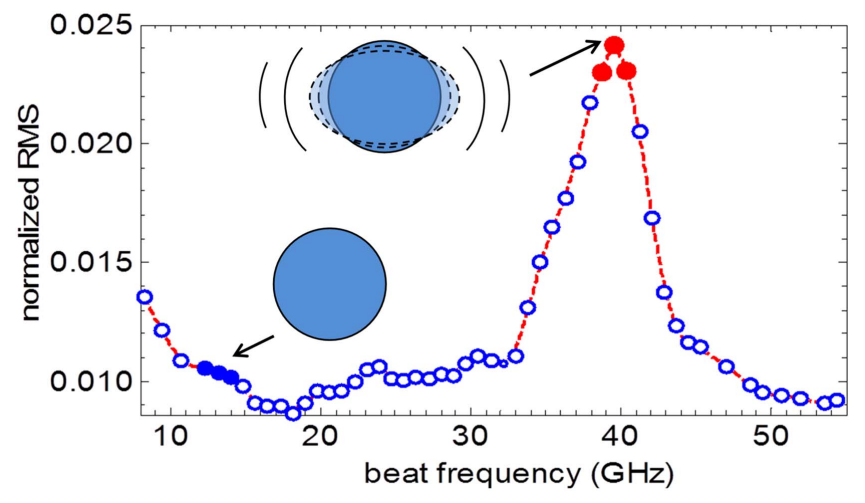

Figure 24. EAR spectroscopy used to probe the elastic vibrations of nanoparticles in a double nanohole. Two trapping lasers are used to produce a beating intensity, which drives the vibrations through electrostriction. Greater noise (normalized RMS variation) is seen at a vibrational resonance.

Based on these early demonstrations, it is envisioned that nanoaperture tweezers will find application in nanoscale assembly, biosensors and new approaches to drug discovery.

\section{Current and future challenges}

Moving nanoaperture tweezers towards their potential first requires an appreciation of the unique benefits of nanoapertures in the context of structured light at the nanoscale. To this end, I outline the main challenges to structured light at the nanoscale for which nanoapertures are particularly well suited to solve.

(1) Apertures couple efficiently to radiation. While plasmonic nanoparticles usually do not achieve even half 
the single channel limit, resonant nanoapertures routinely approach that limit [151].

(2) Local field enhancement. Nanoapertures can provide local fields orders of magnitude higher than the incident field. For example, slits in metal films have been used to confine light to a gap over three thousand times smaller than the wavelength and enhance the surface enhanced IR absorption by $10^{5}$ [152].

(3) Surface sensitivity. Slits have the highest surface sensitivity among common plasmonic structures; higher than single surfaces or thin metal films [153]. Since an aperture can be thought of as a metal-insulator-metal region (like a slit) it is very sensitive to changes in refractive index at the surface.

(4) Heating. Apertures efficiently remove heat because of the surrounding metal film having a high thermal conductivity. For the same local intensity, the heating can be four orders of magnitude smaller than similar plasmonic nanoparticles [154]. (These particles are similar in the sense that they create the same local field intensities on resonance.)

(5) Background interference. Nanoapertures allow for background free operation, limiting signals from the surrounding environment. For example, this has been used to limit the effective probing volume in fluorescence correlation spectroscopy [155].

(6) Polarization control. Nanoapertures allow for polarization control, even allowing for probing chiral lightmatter scattering. Nanoscale chiral light is present in angled slits, for example [156].

(7) Nanoapertures as nanopores. Nanoapertures can be formed as pores to combine with nanopore technologies (such as those envisioned for DNA sequencing).

(8) Scanning nanoapertures. Nanoapertures can be combined with scanning probe microscopies to enable pickand-place nanofabrication and as well as nanopipetting of single nanoparticles from a solution [149].

\section{Advances in science and technology to meet challenges}

Recognizing the potential advantages outlined in the previous section, the key challenge will be in the design and realization of nanoapertures that fully exploit them in many applications, particularly those involving tweezing.

On the design side, numerical and analytic approaches have proved useful in parametric studies of how apertures perform. Ideally, these approaches should be matched with experiments to close the design-build-test-repeat loop.

As with any nanotechnology, there is also the challenge of reliable and scalable fabrication techniques. Template stripping is an example of a promising approach for creating nanoapertures for optical tweezers while limiting the reliance on slow and costly focused ion beam milling [152]. Atomic layer deposition [152] and self-assembled monolayers enable the formation of narrow apertures for maximal field enhancement.

When combining nanoapertures with suspended film (as in nanopores) or fibre-probe technologies (as in near-field tapered probes), the nanofabrication becomes even more challenging and it is not clear if truly scalable nanofabrication will emerge to take this out of the specialist lab and into the general user base.

\section{Concluding remarks}

In summary, nanoapertures provide enhanced functionality for shaping light at the nanoscale because of their strong optical coupling, high local field intensities, high surface sensitivity, low heating, low background noise and degree of polarization control at the nanoscale. Double-nanohole apertures have already been used to analyse proteins, proteinprotein interactions, protein-small molecule interactions, protein-DNA interactions, nanoparticle size/composition, etc. In the future, these apertures can be combined with nanopore and scanning probe techniques to give greater functionality in applications involving nanoscale analysis (e.g., DNA sequencing) and assembly (e.g., placing quantum dots in a plasmonic antenna feed-gap).

\section{Acknowledgments}

Funding has been provided by the NSERC Discovery Grant program. A Kotnala provided the data for the figures. 


\section{Structured light optical tweezers}

\section{Alexander B Stilgoe and Halina Rubinsztein-Dunlop}

The University of Queensland

\section{Status}

The mechanical properties of light have been speculated upon and investigated since Kepler postulated that the shape of the tail of comets depends on the action of sunlight on it in the form of radiation pressure. It took until 1970, when Ashkin observed a significant radiation pressure force on dielectric particles using highly focused laser light, that the first demonstration of optical 3D trapping, an optical tweezers trap or single beam gradient trap, was given [157]. Numerous experiments and developments in the field followed and the phenomenon has found a vast number of applications in colloidal physics, biology and biomedicine. An important capability of optical tweezers is the ability to non-invasively trap and hold in space a single particle using a single beam of light/laser. It has been demonstrated that this technique enables control over the movement of a particle to within a few Ångströms and the measurement of forces acting on the trapped object down to femto Newtons. Optical trapping has been used to manipulate organelles within cells, to study viscoelastic properties, and to study molecular motors, among many other interesting applications. In order to be able to conduct these quantitative studies the traps had to be well characterized and often carefully calibrated.

The use of Laguerre-Gaussian beams improves trap depth along the laser beam propagation axis for small azimuthal mode indices and produces visible orbital motion for large azimuthal modes [158].

The development of holographic optical tweezers closely followed, which enabled studies of hydrodynamic correlations and microscopic artificial crystals. Holographic optical tweezers are used to provide dynamically controlled structured light. In this approach, dynamically modulated phase gradients and multiple traps ensure vastly increased capabilities of optical manipulation [159]. The result of such a light field is shown in figure 25. Large objects can also be manipulated using 'macro tweezers' in which axially structured light is reflected back on itself to create a large dynamical trapping volume, enabling a large cell sized object to be manipulated in 3D using holographic optical tweezers methods [160].

Careful design and fabrication of small structures used in combination with structured light fields can be used to manipulate the surrounding environment, such as the creation of fluid flow or the generation of vortices and torque [161].

The extension of optical tweezers into turbid media requires control of aberrations induced by scattering of the media. Schemes to sample and correct these aberrations have been demonstrated and applied in experiments [162].

Optical fibres can achieve 3D optical trapping using structured light [163]. Dynamical corrections to the wavefront

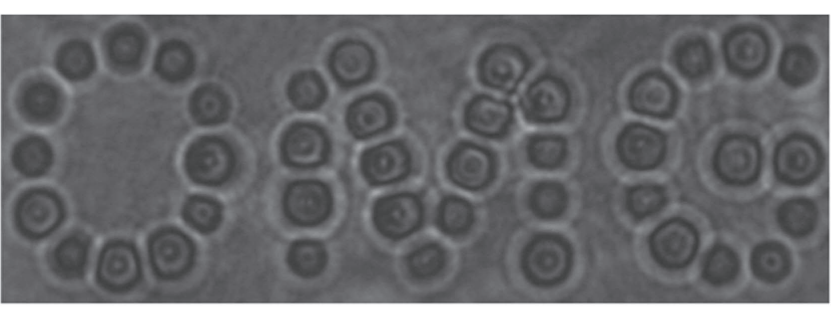

Figure 25. Holographic optical tweezers with 41 traps in a precisely defined pattern.

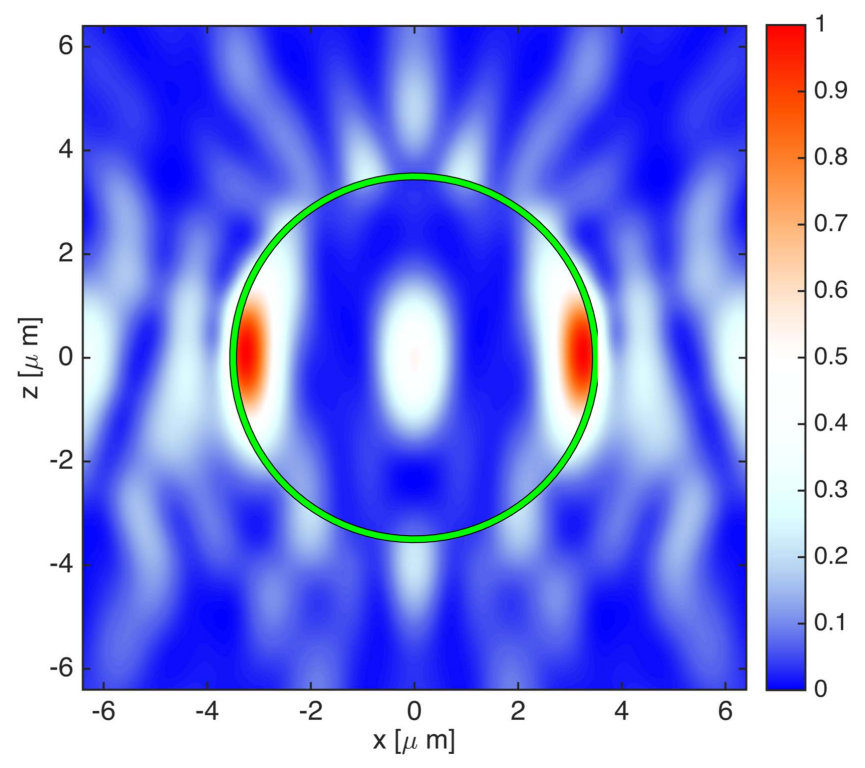

Figure 26. Local field power of the structured light field for enhanced optical trapping of large particles (green circle). Illumination from below.

allow the use of a simple multi-mode fibre to achieve optical trapping [164].

Structured light is also used to simultaneously increase optical trap stiffness and signal-to-noise ratio (SNR). This is achieved by optimizing the incident light field to the geometry of the particle so that small motions lead to large changes in the scattered light [165]. The example of the structured light field scattered by a particle is shown in figure 26 .

\section{Current and future challenges}

Important progress for applying structured light in optical tweezers has been made. The simultaneous sampling of the transmission properties of a system and corrections have been developed. There are still improvements to be made in terms of the speed of generating dynamical structured light for optical tweezer applications. GPU processing techniques using architectures such as OpenGL/OpenCL or CUDA have been essential for the advancement of optical micromanipulation as well as optical trapping and imaging through fibres and turbid media. However, 'real-time' dynamical structured light is only as immediate as the bandwidth of the processing and (in the case of liquid crystal technologies) 
the display step. For example, $30 \mathrm{~Hz} / \mathrm{fps}$ versus $1000 \mathrm{~Hz} / \mathrm{fps}$ can be a factor in the response to dynamical changes in a coupled physical/biological system.

Deep tissue optical trapping requires structured light techniques to be effective by increasing trap stiffness and improving mode shape. Current methods include the use of guide stars to find the scattering tissue phase corrections. This, whilst making deep tissue trapping possible, constitutes an ongoing challenge for the placement of the guide star and the effect that its inclusion has on the system.

Somewhat related to deep tissue trapping and the response to dynamical changes is a challenge associated with the efficiency of dynamical structured light methods as functions of speed, wavelength, and stability. A phase-only shifting device, such as an SLM, suffers from low response times, poor wavelength response and phase-flicker. An amplitude-only device such as a DMD has excellent wavelength response, speed and no phase-flicker, but has inherently low efficiency, limited to approximately $30 \%$ of that of a perfect SLM. A fixed device may be optimized to have excellent wavelength response, stability and fidelity but by definition is inherently unchanging.

The presence of apertures in optical systems disrupts the transmission and transformation of structured light fields. This is especially apparent in optical tweezer systems with highly apertured optical elements to achieve large gradient forces. Therefore, the range of structured light fields practically realizable in optical tweezers are limited. Reformulation of the structured light to be less sensitive to the presence of apertures will improve the production of 'for-purpose' fields in optical trapping experiments. In addition, the high-numerical apertures of optical tweezer systems take the mode shaping problem out of the scalar paraxial wave regime into the time-harmonic vector wave domain [166].

\section{Advances in science and technology to meet challenges}

Continuing improvements in GPU technologies and tools will improve real-time measurement and control of microscopic objects with existing devices. Further development of devices such as DMDs and SLMs will improve the ability to dynamically manipulate sample properties at high efficiency. An ideal device will be comprised of both phase and amplitude quadrature control at high speeds. A future composite device could fulfil this role.

Volume hologram methods will enable greater flexibility and degree of control of structured light, simplifying optical systems and allowing greater integration of optical elements into compact devices. For example, when using a thin device, such as a commercial SLM, the interference in the multiple spots leads to a substantial reduction in efficiency. It is possible to maintain high-efficiency diffraction with a volume hologram. Multi-colour structured light is possible using volume holograms and will lead to greater control of optical micromanipulation as it will enable the construction of complex fields with noninteracting components.

Improvements in modelling optical systems may eliminate the need to use guide stars deep inside tissue so that a broader range of experiments can be carried out. Given the amount of interest in the area of wave diffraction modelling and characterization, new avenues for approaching this problem will develop. The same can be said for non-paraxial modelling of structured light used in optical tweezers.

In an even more speculative idea, we could imagine an illumination source combining a dynamical wave shifting device to convert a source laser into a perfect Huygens-type light source where high-intensity light gradients appear on demand. It would simultaneously remove many of the optical elements used today and miniaturize the experimental apparatus such that it could be utilized in lab-on-a-chip devices. The resolution of such a device will be a limiting factor for the production of large intensity gradient structured light from a plane source.

\section{Concluding remarks}

Structured light has been well integrated into optical tweezer experiments. A number of challenges still remain with regards to the speed, efficiency and range of applicability. The fulfilment of these challenges will ensure continued development of the field and lead to a number of scientific discoveries by leveraging the promise of non-contact manipulation of optical tweezers and the flexibility and level of control offered by structured light methodologies. 


\section{Quantum information processing with structured light}

Jacquiline Romero and Andrew G White

University of Queensland

\section{Status}

The spatial structure of light most often refers to the amplitude and phase modulation of the field on the transverse plane-the transverse spatial mode-but can also refer to other properties such as the direction of the oscillation of the field - the polarization mode. In this short review, we focus mainly on the transverse spatial mode (see figure 27). Encoding information, which is equivalent to the generation of the different transverse modes, has moved from the use of static elements to the use of dynamic elements. The use of programmable devices, such as SLMs, deformable mirrors and DMDs, is becoming more common as an alternative to the use of fixed holograms and mode converters, consisting of refractive optical elements such as cylindrical lenses. Optical elements also work in the reverse direction; hence the readout of information is also well developed using the same elements used for encoding combined with appropriate detectors (see figure 28).

Quantum information processing, or the manipulation of information between encoding and readout, comes in many forms. The simplest is quantum communication, which requires encoding and decoding with minimal manipulation inbetween. Much has been done in this area (see the review in section 18). The most difficult is universal quantum computation, and the use of structured light in this field is still in the juvenile phase. For example, quantum teleportation, which is recognized as a building block for universal quantum computation, was only recently shown in hybrid spin and OAM states of single photons (associated with both the polarization and transverse modes) [167]. Intermediate between quantum communication and universal quantum computation is quantum simulation, and this is an area where the naturally high-dimensional nature of structured light can be useful.

The Gaussian family of transverse modes provide a straightforward, orthogonal basis to access higher dimensions. Extending quantum information processing to qudits (d-level systems) is motivated by practical advantages such as increased information capacity, resilience to noise and improved security.

\section{Current and future challenges}

Detection efficiency is an issue that all optical implementations of quantum information processing confront. Highefficiency (above 90\%) superconducting single-photon detectors are available, but they require very low-temperature operation. These detectors are usually fed by single-mode fibres that transmit only the fundamental Gaussian mode. Hence, an efficient method is needed to transform the mode resulting from the processing task to the fundamental

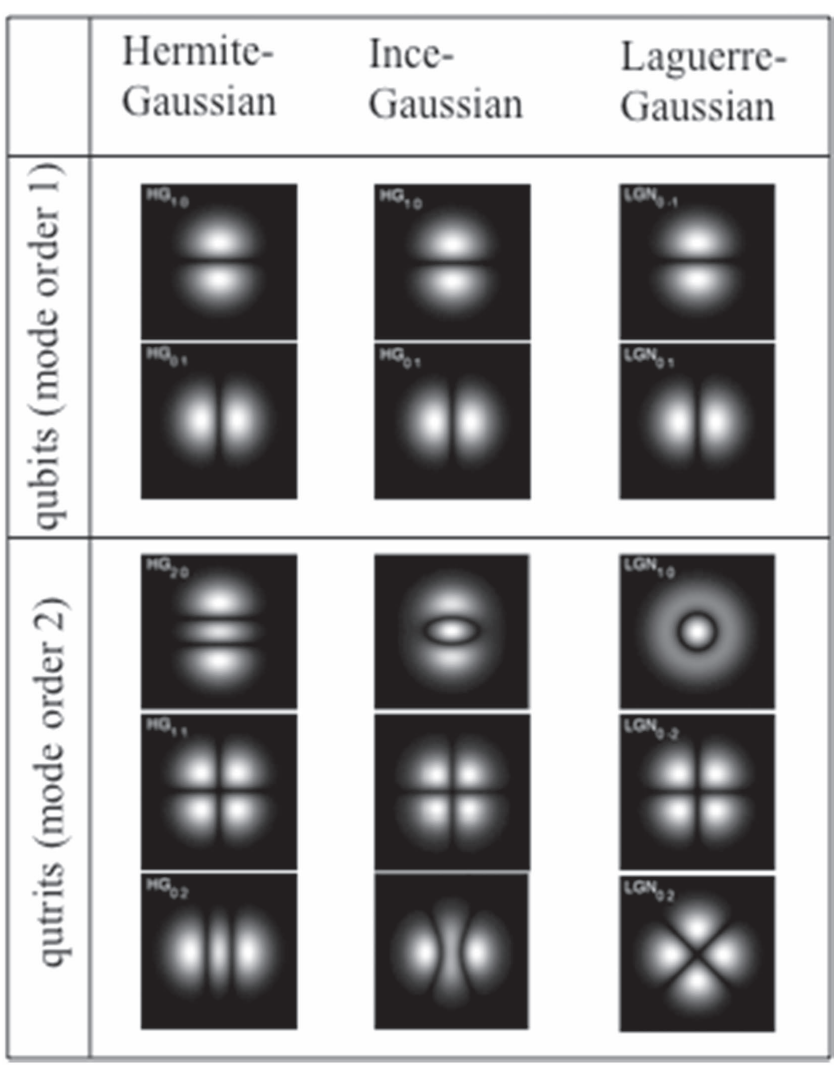

Figure 27. Gaussian family of modes. Different orders provide a natural basis for qudit quantum information processing.
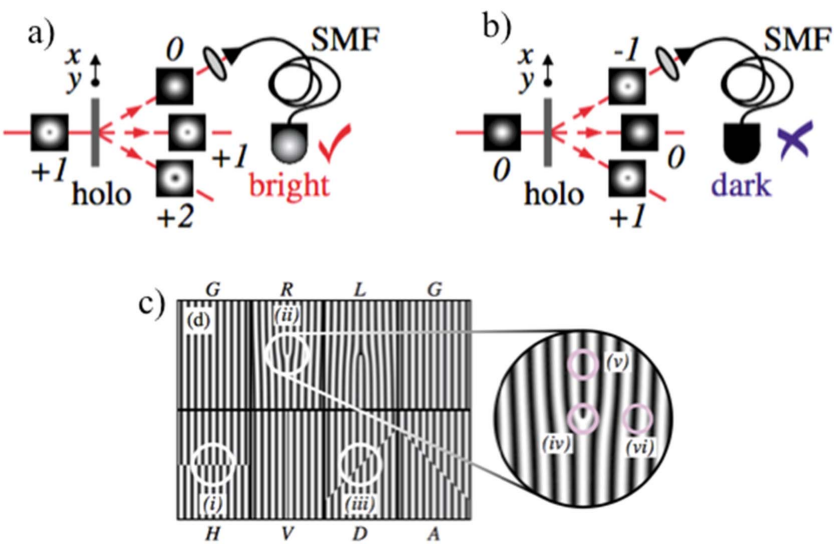

Figure 28. Scheme for quantum state tomography of qutrit using holograms. The target mode couples to the detector (a), while any other mode is rejected (b). The eight-segment analysis hologram need not measure all three-mode superpositions; it is enough to measure two-part superpositions. $\{\mathrm{G}, \mathrm{R}, \mathrm{L}, \mathrm{H}, \mathrm{V}, \mathrm{D}, \mathrm{A}\}$ correspond to the main spatial mode analysed by that segment. The positions (i)-(iii) and and (iv)-(vi) correspond to degenerate and nondegenerate qutrits, respectively. Reprinted with permission from [175]. Copyright 2004 by the American Physical Society.

Gaussian mode. The transformation is commonly achieved with planar holograms (see figure 28), but this is not highly efficient and provides only an approximate transformationalbeit $90 \%$ and above-if both amplitude and phase are not simultaneously controlled [168]. To circumvent the need for this conversion, optical fibres that can support structured light 
can be used. However, these types of fibres are not widely available, and even then support only a few modes [169].

Quantum computation with qubits, such as the polarization of single photons, is well understood in theory. Within the circuit model of computation, universal gate sets for qubits are known, e.g. any operation can be reduced to a combination of the Hadamard, phase rotation and CNOT gates. More importantly, these gates can be implemented with linear optics [170]. Universal quantum computation for qudits require arbitrary $d$-dimensional rotations and a generalized CNOT operation (CINC or SUM in the literature) [171]. However, experimental implementations of these operations are scarce because of the difficulty, and more importantly because - at least in the circuit model of quantum computation-using qudits instead of qubits offers no known computational advantage [172].

By contrast, there is an advantage in using structured light within the cluster model of quantum computation, where larger cluster states are needed for longer computations. Cluster states can be created deterministically for continuous variables, e.g. in squeezed light, whereas they can only be created probabilistically with qubits [173]. Involving more transverse spatial modes can increase the size of the cluster state, and therefore the information processing capability. Sources of such clusters of transverse spatial modes have not been developed yet, and even if they are, the coherent, nonlinear operations required are difficult to perform in a lossless manner.

\section{Advances in science and technology to meet challenges}

Programmable volume holographic elements with a high mode conversion efficiency have the potential to revolutionize quantum information processing with structured light. Such elements will have the capability of transforming between the different modes within the high-dimensional state space. This has the obvious benefit of increasing both generation and detection efficiencies, but more importantly can serve as convenient single qudit gates to achieve single qudit manipulation. Research in volume holography is often geared towards storage and projection [174], and much work is needed for quantum information processing using these devices.

Universal quantum computation with qudits remains to be formulated and demonstrated, but simpler tasks such as quantum bit commitment and quantum state tomography have been achieved [175]. One realization that applies to tomography is that measuring $d$-superposition states is not necessary [175], making the holograms less complicated. If universal quantum computation with qudits can be formulated such that arbitrary rotations within different subsets of the $d$ dimensional space are enough, the manipulation can also be made simpler. But as it is, there are more promising platforms for universal quantum computation, e.g. cluster state with polarization qubits, therefore, from the theoretical side, the community can instead focus on algorithms that naturally make use of qudit encodings. Examples include the quantum Fourier transform, magic state distillation, and simulations of quantum field theories [176].

Information encoded in light is stable because there is very little intrinsic decoherence at electromagnetic frequencies. Whilst a boon for encoding, this is a bane for the processing of information. Because of the lack of photonphoton interaction in linear optics, current two-qubit gates work only probabilistically. Nonlinearities sensitive to the single-photon level have only recently been achieved in photonic nanostructures, e.g. using quantum dots in a photonic crystal waveguide [177, 178] or micropillar cavity [177]. It is likely that nonlinearities will also play a role in implementing two-qudit gates and also offer better storage since memories are inherently multilevel. Continued advance in this area can lead to more efficient quantum information processing architectures in the future.

\section{Concluding remarks}

Quantum information processing with structured light is a natural route to higher-dimensional quantum computation. Protocols such as teleportation, quantum state tomography and quantum bit commitment have been shown, but more complicated processes await demonstration. On the theoretical front, more motivation is needed to inspire experimental implementations of quantum computation with structured light. On the technological front, qudit manipulation is the most salient issue that needs to be addressed. Programmable volume holographic elements can fill this gap, but research in this area in connection with quantum information processing is limited. An indirect, but nonetheless promising approach could be the integration of transverse spatial modes with other, more often used degrees of freedom such as polarization and path (as in waveguides). Optical nonlinearities from nanostructures can also usher in photon-photon interactions specific to structured light and that will open up novel qudit architectures.

\section{Acknowledgments}

The authors thank Timothy Ralph, Nicolas Menicucci, and Gavin Brennen for useful discussions. 


\section{Quantum communication using structured light}

\section{Robert Fickler}

University of Ottawa

\section{Status}

Quantum information science has numerous potential applications, from allowing for computational speed-up to achieving unconditionally secure communication. It has pushed the limits of our understanding of quantum physics as well as our technological ability to control quantum systems. When it comes to the distribution and communication of quantum information over long distances, only photons can be considered as realistic candidate carriers of quantum information. Moreover, photonic quantum experiments are already in the process of opening up a novel, promising field of quantum information science, namely high-dimensional encoding (e.g. see section 17). By moving beyond qubits to high-dimensional states to encode quantum information, such so-called qudits pave the way to novel fundamental tests and enhancing many quantum information protocols, such as superdense coding or quantum key distribution (QKD) schemes. One of the most rapidly evolving fields to physically implement such an encoding scheme is the use of the transverse spatial mode of light and its nearly arbitrarily complex structure.

Shortly after the first experimental demonstration of entanglement between light structures (which intended to show entanglement of OAM [58]) the first high-dimensional experiment and its application to quantum cryptography was demonstrated. Since then the technology to generate, manipulate and detect quantum states encoded in the structure of light has advanced, and so have demonstrations of their use in quantum communication. Recently, experimental demonstrations have further pushed the limits on the dimensionality of entanglement [179] and the distance over which such states can be distributed [180]. In particular, much attention has been paid to high-dimensional quantum cryptography using structured light, due to its higher key rate and better noise resistance [181].

However, compared to the mature technology of commercially available 2D quantum communication systems, many challenges are still waiting to be solved for structured light. Ongoing efforts to meet the requirements of quantum schemes in efficiency and quality will be beneficial in other applications of structured light, such as classical information encoding, enhanced imaging or optical tweezers. Similarly, novel findings from these fields will speed up the progress of quantum communication science.

\section{Current and future challenges}

Quantum information schemes using complex light modes have increasingly attracted attention and advanced in recent years. The fundamental reason for this is the laboratory realization of a high-dimensional quantum state. Another important reason is the fast progress in the generation and detection of structured photons with matured technologies, such as SLMs or single-photon sensitive cameras. Although the versatility and efficiency of such devices already meet the required standards, their usefulness in future applications or novel proof-of-concept experiments is limited to modulation frequencies of at most a few $\mathrm{kHz}$ [181]. However, a recently developed technique to efficiently sort structured light modes has helped to overcome some major obstacles [182]. With it, and its subsequent enhancements, near-perfect deterministic detection with multiple outcomes has become possible. Similarly, the sorter, if used in reverse, generates structured photons; thus, it translates the modulation frequency problem for generating structured photons to the problem of switching between different paths, a task which can be carried out at very high speed. The application of this technique in quantum experiments has already begun, and will advance the field of high-dimensional quantum communication considerably.

In order to fully take advantage of the structured photons and the associated high-dimensional state space, an important device is still missing: a device that is able perform any modulation (unitary operation) of the qudit state in a controlled and efficient manner. Such a device, often called a multiport, would allow quantum operations in the highdimensional space and could therefore be used to perform quantum computational tasks even on a single carrier. Additionally, it would improve quantum communication schemes by enabling (in combination with the aforementioned sorter) efficient detection in all mutually unbiased bases, thereby advancing high-dimensional quantum state analysis and QKD schemes.

Another important task will be the efficient and undisturbed long distance distribution of the structured photons. Here, fibre and free-space links are facing different challenges. Standard fibres that support multi-modal structures must compensate for strong intermodal coupling that otherwise limits their practicality. Custom-made fibres [183] overcome this limitation (see figure 29(a)) but still ought to be considered ongoing research, especially for larger dimensions and in quantum experiments. In free space, atmospheric turbulence distorts the spatial structure and thus prevents errorfree transmission. Although intra-city links of up to $3 \mathrm{~km}$ have been shown to work without active compensation for such disturbances [180], compensation will be required to enhance performance, and enlarge communication distances, and are therefore an inevitable next technological challenge to be tackled (see also section 19). While both options show tolerant rates of loss upon propagation, the distance crucially limits the maximal detection efficiencies for free-space links due to enlarged modes.

However, generating, manipulating, distributing and measuring spatial structures of quantum states is only one side of the coin: entangling photons and distributing entanglement are other challenges yet to be solved. The most common way of generating high-dimensional entanglement relies on the statistical process of parametric down-conversion, in which neither the dimensionality of the entanglement, nor the number of entangled photons can be easily tailored at will. 
a)
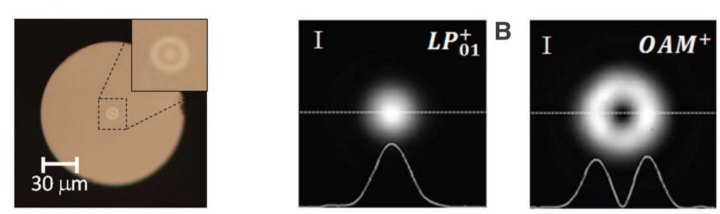

b)

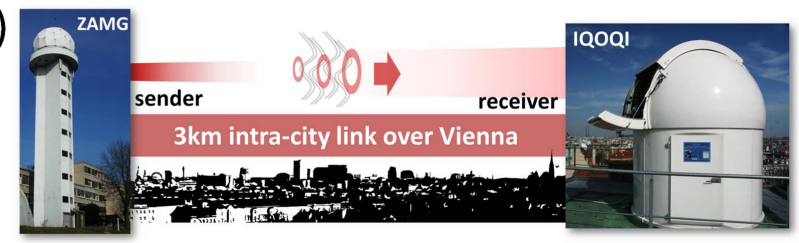

c)

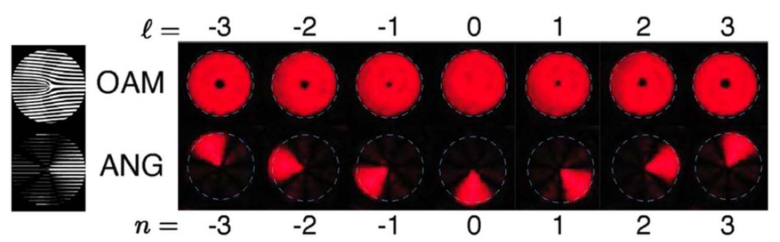

Figure 29. (a) Structured light supporting fibre and measured output modes. From [183]. Reprinted with permission from AAAS. (b) First outdoor free space link distributing structured photon entanglement [180]. Copyright 2015 National Academy of Sciences, USA.(c) Two mutually unbiased bases of seven-dimensional quantum states used to implement QKD. Reproduced with permission from [181]. Copyright 2015 IOP Publishing Ltd and Deutsche Physikalische Gesellschaft.

Better control would certainly be necessary for a quditboosted quantum network. In addition, the distribution of high-dimensional entanglement of structured photons would be required. Again, the direct distribution of entangled photons over long distances has yielded promising first results, but needs to be improved. Additionally, a key feature in quantum information, quantum teleportation of a highdimensional state, is still missing even in the form of a labscale demonstration. In combination with high-dimensional quantum memories and coupling to local quantum processing units such as ions, which have both been demonstrated recently [184, 185], a global quantum network including possible quantum repeaters might be conceivable.

\section{Advances in science and technology to meet challenges}

The huge advance of quantum information science using structured light has mainly been driven by improved technologies, pointing to the necessity of novel, improved devices to further advance the field. Future technologies allowing for faster modulation of the complex spatial structure of light will improve quantum communication schemes, e.g. by active compensation of disturbance in free space and fibre transmission, larger secure key rates or enhanced discrimination of qudit states. In addition, single-photon sensitive cameras with extreme temporal resolution are promising candidates to lift the restrictions that single-pixel detectors have on multidimensional detections.

Along with such promising technological progress, the theoretical framework has to be further investigated and developed. Security proofs and novel protocols for highdimensional QKD schemes might be further optimized, computational algorithms have to be adapted or developed to fully account for the large state space attached to a single photon, and error-correction schemes for high-dimensional quantum computation have to be investigated deeper.

Apart from the promising applications, it will also be interesting to see if structured photons and their highdimensional quantum states can be utilized to investigate the novel fundamental properties of complex high-dimensional quantum states, which allow for new types of quantum communication schemes. An example, layered quantum communication, was recently discussed in connection to a multipartite entangled experiment using structured photons [186]. If both the number of photons and the number of dimensions exceed two, the entanglement can be shared asymmetrically, which enables one to secure information in different layers between the involved parties.

Lastly, it should be mentioned that novel ways to couple the spatial structure to other degrees of freedom (DOF), such as the temporal or polarization DOF of light, may also be beneficial for quantum communication tasks. The latter, for example, leads to a rotational invariant pattern, lifting the requirement of a shared rotational reference frame [187] (see also section 8).

\section{Concluding remarks}

Since the very first demonstration of quantum entanglement, structured photons have been used to apply and improve quantum communication schemes. Along with novel fundamental discoveries, e.g. multipartite high-dimensional entanglement or quantum memories for structured photons, and rapid technological progress, the field can be considered a major component in a possible future quantum network boosted by high-dimensional quantum states.

\section{Acknowledgments}

RF acknowledges helpful discussions with M Krenn and N Tischler and financial support by a Banting postdoctoral fellowship of the Natural Sciences and Engineering Research Council of Canada (NSERC) and the Canada Excellence Research Chairs Program. 


\section{Classical optical communication systems using structured light}

\section{Alan E Willner and Guodong Xie \\ University of Southern California}

\section{Status}

The exponentially growing demand for data capacity has always motivated the optical communications community to continually explore methods that can achieve higher system capacity and spectral efficiency. Historically, multiplexing of multiple independent data streams has provided significant enhancements. Examples include polarization division multiplexing (PDM) and wavelength division multiplexing (WDM). Another approach to increase capacity could be space division multiplexing (SDM), in which multiple beams are simultaneously transmitted and can occupy a unique position in the spatial domain [63]. A subset of SDM is modedivision multiplexing (MDM), in which each beam is transmitted on a unique spatial mode. Moreover, the modes are orthogonal to each other, enabling efficient (de) multiplexing and low intermodal crosstalk; note that SDM is compatible with PDM and WDM. One MDM approach is the use of an orthogonal set of structured beams, such as OAM, for which each beam has a helical transverse phase structure of $\exp (i \ell \varphi) ; \varphi$ is the transverse azimuthal angle and $\ell$ is an unbounded integer denoting the OAM state number. OAM beams with different $\ell$ values are mutually orthogonal, allowing them to be multiplexed together along the same beam axis and demultiplexed with low inherent crosstalk (see figure 30) [188]. Classical optical systems have been demonstrated with the following selected results: (a) $\sim 2$ Tbit $\mathrm{s}^{-1}$ free-space transmission over $1 \mathrm{~m}$ using OAM and PDM [189], (b) $\sim 100$ Tbit $\mathrm{s}^{-1}$ free-space transmission over $1 \mathrm{~m}$ using OAM, PDM, and WDM [190], (c) $400 \mathrm{Gbit} \mathrm{s}^{-1}$ freespace transmission over $120 \mathrm{~m}$ using OAM [190], (d) 2 Tbit $\mathrm{s}^{-1}$ using OAM and WDM over $\sim 1.1 \mathrm{~km}$ of specially fabricated 'vortex' fibre for which the OAM modes exhibit relatively little power coupling as they propagate [191] and (e) using OAM to send data between two sites over $143 \mathrm{~km}$ [192]. We note that there are other orthogonal modal basis sets that could also be used for multiplexing, especially in free space. However, OAM modes may offer the potential advantage of being conveniently matched to many optical subsystems due to their circular symmetry.

\section{Current and future challenges}

Several key challenges exist when implementing a classical communication system that is based on uniquely structured multiple OAM beams. In free-space transmission, the following are some critical issues: (1) divergence: an OAM beam diverges faster than a regular fundamental Gaussian beam, giving rise to increased power loss for a limited-size receiver aperture; (2) signal power: to recover data from the multiplexed beams, one needs to recover both their phase

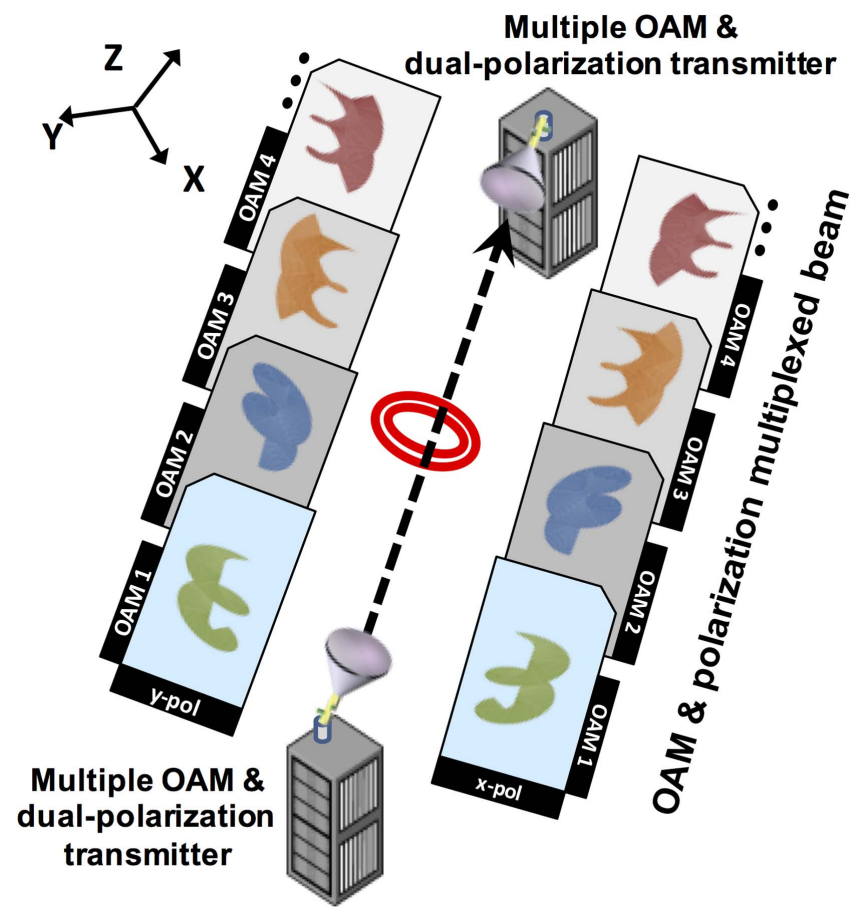

Figure 30. Concept of free-space transmission with a single transmitter and receiver aperture pair, in which each polarization contains multiple OAM-multiplexed beams.

information and signal power simultaneously. The phase information enables the efficient separation of the multiplexed beams while the signal power guarantees enough SNR for data recovery. Unfortunately, the amount of phase change per unit area for an OAM beam is greatest at the beam centre, where there is also lower signal power. Therefore, the design trade-off between capturing the beams' phase information and intensity information should be carefully considered to ensure a high SNR and low modal crosstalk. Issues (1) and (2) combine to require a relatively larger aperture size and a more limited total transmission distance [16]. (3) Pointing accuracy: orthogonality between OAM beams is ensured when all beams are co-axially propagated and the receiver is perfectly aligned on-axis with the beams [16]. However, any misalignment among the beams or between the beams and receiver can degrade the orthogonality due to a phase mismatch and cause power to be coupled into other modes, i.e. severe intermodal crosstalk. Therefore, systems should be well aligned and might require active pointing and tracking to ensure low crosstalk. (4) Turbulence: inhomogeneity in the temperature, pressure and composition of the atmosphere can lead to variations of the refractive index along the transmission path [193]. Given that there are multiple co-propagating beams and that the orthogonality depends on the phase-front, such atmospheric turbulence can cause both degradation of a single OAM channel and an increase in the intermodal crosstalk among different OAM channels.

In fibre-based OAM systems, a few key challenges include: (1) mode launcher: accurate and careful mode launching of an OAM mode into any type of fibre is required, since any misalignment may cause power loss and power 
coupling into the wrong modes. (2) Mode coupling: whether using vortex fibre, few-mode fibre, or any other type of fibre, a critical issue is the maintenance of power in the respective modes during propagation. In all the above free space and fibre challenges, additional signal processing may be required for data recovery in the presence of intermodal crosstalk (see next section).

\section{Advances in science and technology to meet challenges}

The following approaches may provide potential solutions for the above-mentioned challenges: (1) beam structuring: to deal with the trade-off between recovering maximum phase information and maximum signal power, novel approaches should be pursued to optimize both of these parameters. For example, one might pursue novel types of structured beams that have most of the phase change and intensity concentrated at the same location. Moreover, quasi-beam-forming could also be potentially helpful for achieving this goal. (2) Pointing and tracking: since misalignment is deleterious to the orthogonality and modal purity of transmitted beams, highly accurate pointing, acquisition, and tracking systems with a feedback loop that is faster than any possible system changes should be explored. (3) Crosstalk mitigation: in many free space and fibre applications, crosstalk among modes may appear. Reports have shown that adaptive optics and electronic digital signal processing (DSP) approaches can reduce the crosstalk, such as: (a) adaptive optics: the phase distortion of the beams can be partially compensated in the optical domain using adaptive optics (figure 31) [193], in which a wavefront sensor measures the wavefront distortion of the OAM beams and a tunable phase hologram induces a conjugate phase on all the beams; (b) DSP: advanced electronic digital post-processing using channel equalization DSP algorithms that can mitigate the modal crosstalk degradations such that recovering multiple beams can simultaneously provide mutual information for 'unwinding' some of the crosstalk [190]. Important future considerations on crosstalk mitigation could focus on the potential advantages of: (i) combining optical and electronic compensation techniques, and (ii) combining multiple OAM beams on each of the multiple aperture pairs (i.e. multiple-input-multiple-output). (4) Obstruction tolerance: obstructions present a critical limitation in many free-space optical links, and this is even more so for OAM systems for which the obstruction can disrupt the phase and cause intermodal crosstalk. It might be important to explore beams whose phase and amplitude could survive a partial beam obstruction. At present, 'propagation-invariant' structured Bessel beams have shown promise, but only over limited distances [194]. Owing to their propagation-invariance property, Bessel beams are able to 'self-heal', i.e. reconstruct their complex field when subjected to an obstruction or distortion. Therefore, it may be possible to develop new types of structured beams suitable for tolerating

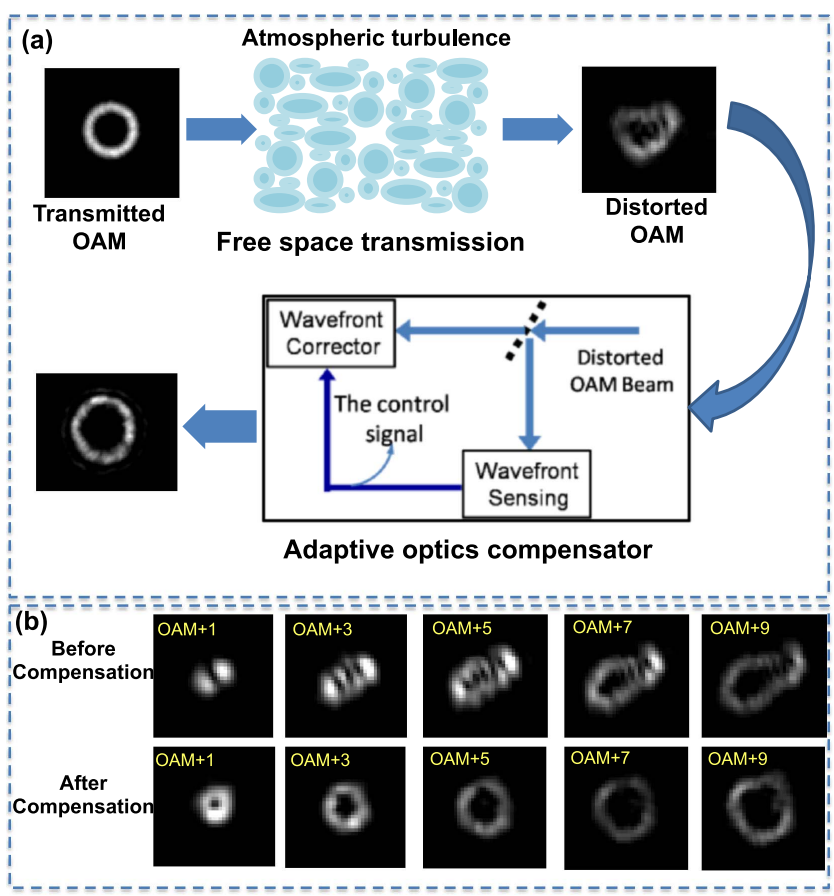

Figure 31. (a) Concept of using adaptive optics for the wavefront correction of OAM beams. (b) OAM $+1,+3,+5,+7$ and +9 before and after compensation. Reproduced with permission from [190]. Copyright 2015 The Optical Society.

partial beam obstructions. (5) Cost-effective components: future deployment of classical optical communication systems using OAM will likely require the development of many types of components [195]. In general, such components should exhibit high performance, good dynamic range, and low cost.

\section{Concluding remarks}

Classical optical communication systems using the multiplexing of structured light represents a young sub-field that has a rich set of issues to explore, including technical challenges and potential application opportunities. Will OAM multiplexing be deployed in future communication systems? Right now, it is hard to predict, but the prospects are indeed exciting in terms of the potential systems advantages. Moreover, it would not be surprising if the innovations and advances in OAM-based communication systems find applications in other areas.

\section{Acknowledgments}

We acknowledge the generous support of Air Force Office of Scientific Research, Intel Labs University Research Office, National Science Foundation, and NxGen Partners. 


\section{Structured electron matter waves}

\section{Benjamin McMorran}

University of Oregon

\section{Status}

Electron matter waves can be sculpted to have non-trivial wavefront topology and intensity distributions. These structured electrons provide novel capabilities for electron microscopy and spectroscopy, as well as new insights into basic physics. The ability to coherently manipulate free electron wavefunctions into arbitrary shapes is enabled by new tools in electron optics such as holographic diffractive optics. For example, nanoscale diffraction holograms were recently used to shape freely propagating electrons into quantum vortex states [6, 196]. Analogous to an optical vortex, an electron vortex is a helical de Broglie matter wave that carries quantized OAM. These and other types of structured electron wavefunctions show promise for nanoscale imaging and spectroscopy. Challenges, advancements, and future possibilities of this work are outlined here.

Both electrons and light evolve in free space according to formally equivalent wave equations: the Helmholtz equation in the case of light and the time-independent Schrodinger equation in the case of electron matter waves. Thus, any type of structured lightwave that solves the Helmholtz equation, such as OAM beams, can also be created in a non-relativistic, mono-energetic electron wavefunction. With this in mind, several research groups independently demonstrated electron OAM beams [6, 196, 197] by coherently sculpting free electron matter waves into helical structures. Other types of structured electron matter waves have since been demonstrated, such as Bessel [198] and Airy beams [199].

While the electron can be manipulated in analogous ways to the photon, its rest mass and charge provide unique applications for structured electron wavefunctions that are inaccessible with structured light. For example, charged electron OAM beams can be focused down to Ångström length scales [200, 201], and display unusual interactions with magnetic fields [202, 203] and chiral materials [204]. Unlike photon OAM, electron OAM can directly excite dipole transitions in atoms through the Coulomb interaction. Not only can this lead to new selection rules in electron-atom interactions, but it can also be used for magnetic dichroism at atomic length scales [205-209]. Structured electrons have also recently been used to enhance phase contrast in transmission electron microscopy (TEM), providing the ability to image electron-transparent objects at small beam doses and improved spatial resolution [210].

\section{Current and future challenges}

The physics of structured electrons presents unique experimental challenges. For example, in proposed electron orbital dichroism experiments, a high-intensity electron vortex beam with precise phase must be focused down to the size of a single atom and positioned with sub-Ångström accuracy [205-209]. Furthermore, the electron wavelengths are typically 100000 times smaller than optical wavelengths, and interact much more strongly with matter. Special tools developed to sculpt electrons can be divided into two categories: holographic and non-holographic methods.

Non-holographic electron wavefront sculpting techniques are borrowed from optics. Spiral phase plates [197] and cylindrical lens mode converters [211] have both been developed for electrons. Uniquely, electron OAM can also be generated upon propagation through magnetic spiral phase plates provided by the field of a magnetic monopole [212], which can be approximated by the field surrounding the tip of a magnetic needle [213, 214]. The advantages of such non-holographic methods for electron vortex beams is that they build on existing electron phase plate technology and can be extremely efficient, converting nearly all of the incident electrons into OAM beams. Another advantage of some of these techniques is that the OAM can be dynamically controlled, which enables detecting small OAM-dependent signals using lock-in techniques. However, as in light optics, it is extremely difficult to achieve pure OAM states with precise phase using these non-holographic methods.

Holographic electron wavefront sculpting utilizes nanoscale diffractive optical elements [6, 196, 215-218] to coherently manipulate electrons. In principle, diffraction holograms offer a way to sculpt the phase of structured electrons with higher fidelity than non-holographic methods, since the phase can be precisely controlled by the position of grooves or slits in a mask. However, when using nanofabricated diffraction holograms in TEMs, the diffraction angle of picometre-wavelength electrons from fabricated features in the holograms is quite small. Thus, it can be difficult to isolate a particular phase-engineered diffracted beam from amongst the rest of the diffraction orders. Furthermore, only a fraction of the original electron-beam gets diffracted.

A challenge and opportunity for holographic electron optics is presented by a need to tune the electron phase structure

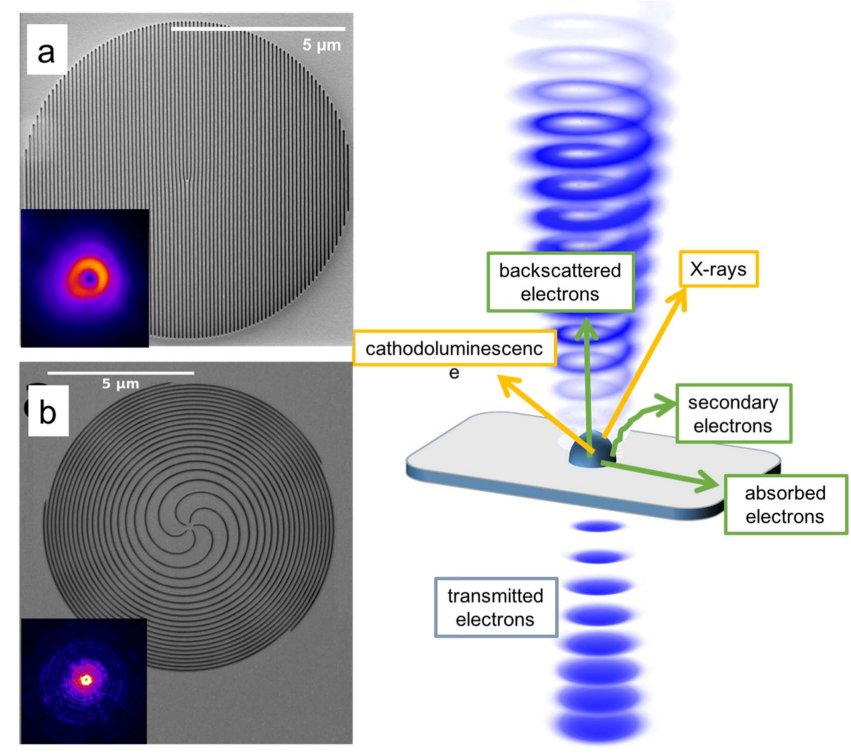

Figure 32. Sculpted electrons, such as electron vortex beams, can interact with matter in new ways due to the non-trivial topology of the wavefront. Electron vortex beams are produced using nanofabricated diffraction holograms. Panels $(\mathrm{a}, \mathrm{b})$ reproduced with permission from [216]. Copyright 2014 IOP Publishing Ltd. 
Table 1. A list of challenges and solution strategies for structuring electron matter waves.

\begin{tabular}{lll}
\hline Challenge & Strategy & Status \\
\hline Precisely structure phase & Diffractive, not refractive, optics & Sculpt phase with 100 mrad precision \\
Maximize beam intensity & Transparent blazed phase gratings & Diffracting up to $80 \%$ of incident beam \\
Control intensity profile & Apodization by varying diffraction efficiency & Demonstrated pure Gaussian modes \\
Atom-sized beam focus & Large area hologram & $\varnothing 50 \mu \mathrm{m}$ area \\
& Aberration correction & $\rightarrow 1.2 \AA$ probe \\
Isolate structured beams & Nanoscale grating & $80 \mathrm{~nm}$ pitch \\
& Blazed or sinusoidal & $\rightarrow 25 \mu$ rad (63 nm apart) \\
Stability & Maximize lifetime & Weeks of use before cleaning \\
Dynamic tunability & Adjustable electron wavefront modulator & Digital pattern generator \\
\hline
\end{tabular}

dynamically. Nanofabricated diffraction holograms are not adjustable, and can only be used to imprint a static phase distribution onto the beam. However, in many current and future applications, it is desirable to have an electron optical equivalent of an SLM, which can be re-programmed to rapidly adjust the spatial phase of the electron beam at will. Such a device, an electron wavefront modulator, would provide the ability to compensate for unwanted fluctuating aberrations inherent to electron optical systems, or to dynamically adjust the electron wavefronts to suit the immediate needs of an experiment. For example, in electron orbital dichroism experiments, a programmable wavefront modulator could be used to rapidly switch the OAM of the probe beam without changing any other imaging conditions, dramatically easing experimental difficulty.

\section{Advances in science and technology to meet challenges}

As in optical computer-generated holography, the design of a diffractive hologram is computed from the simulated interference pattern between a desired electron wavefunction and a reference wave. When the pattern is fabricated into a mask and then illuminated by a spatially coherent electron beam, the time-reverse of the simulation occurs in actuality: electrons diffract from the structure and evolve such that the desired wavefront is reproduced. The location and curvature of the lines in the hologram encodes the phase structure of the diffracted beam. For example, forked linear gratings (figure 32a) and spiral circular grating (zoneplate, figure $32 \mathrm{~b}$ ) give rise to helical electron wavefronts with characteristic dark spots at the center of the beams.

The nanofabricated hologram is installed in an electron microscope's beam-forming aperture, and a Fourier transform of the hologram is produced at the specimen plane of the instrument. Thus, minimizing the grating periodicity of the hologram maximizes the separation between diffracted electron beams. Maximizing the hologram area provides a larger numerical aperture for smaller focused spots.

We have developed a fabrication technique for nanoscale material gratings that produce high-intensity, isolated diffracted electron beams with precisely engineered wavefront topology. We typically mill hologram patterns onto $20-150 \mathrm{~nm}$ thick metal-coated silicon nitride membranes. These are mechanically robust, thermally stable, and commercially available, and can be transparent to electrons. We use direct, low-level control of a focused ion beam to sequentially mill grooves with $80 \mathrm{~nm}$ spatial periodicity covering $50 \mu \mathrm{m}$ diameter areas. We also control the depth, width, and shape profile of the groove in order to maximize the diffracted intensity into particular orders, for example using blazed grating or sinusoidal surface profiles to suppress unwanted diffraction orders [216]. In an aberrationcorrected scanning TEM, nanofabricated holograms can produce multiple structured probes with 1.2 Ångström diameters [200, 201]. Table 1 summarizes these advancements.

While a dynamically programmable, reconfigurable electron wavefront modulator does not yet exist, advancements in the semiconductor industry may point a path to this. A CMOS-based digital pattern generator module for electrons has been developed [219] for use in reflective electron beam lithography (REBL) [220]. The pattern generator module consists of an array of micron-sized electrostatic mirrors for electrons that selectively reflect or absorb electrons. The device is currently optimized for use as a digital binary mask to imprint an intensity pattern onto an electron beam, yet with improved spatial and voltage resolution, this technology could be used to diffract electrons, providing an SLM-like device for electrons.

\section{Concluding remarks}

Nanoscale diffraction holograms are a new technology for coherently sculpting electron matter waves with unusual properties. For example, electron vortices demonstrate that particles with charge and mass can occupy quantized orbital states in free space with a simple physical explanation in terms of collections of skewed trajectories [196, 221]. Several research groups are utilizing structured electron wavefunctions in electron microscopes as a new way of investigating chirality, magnetism, and structure of materials in new ways at the nanoscale. Future improvements in electron diffractive optics will foster a new era of electron microscopy.

\section{Acknowledgments}

This work was supported by the US Department of Energy, Office of Science, Basic Energy Sciences, under Award \# DE-SC0010466. The author gratefully acknowledges the use of CAMCOR facilities, which have been purchased with a combination of federal and state funding. 


\section{Structuring light in time}

\section{Andrew M Weiner}

Purdue University

\section{Status}

Methods allowing the transformation of mode-locked laser pulses into nearly arbitrarily shaped, user defined waveforms were introduced more than two decades ago. Commonly termed pulse shaping [222, 223], these methods rely on spectral dispersion to spatially separate, and subsequently recombine, the frequency components making up a broadband input pulse. While separated, the phase and amplitude (and sometimes the polarization) of the dispersed optical frequency components can be manipulated in parallel using various SLM technologies. The result is Fourier synthesis: after the frequencies are put back together, the output waveform is determined by the inverse Fourier transform of the spatial pattern transferred onto the complex optical spectrum (figure 33). Pulse shaping has found widespread applications both in technology and ultrafast optical science. User defined ultrafast waveforms have been used to explore coherent laser control over photochemical reactions and quantum mechanical processes, in the implementation of multi-dimensional optical spectroscopies, and in compressing pulses to durations approaching the oscillation period of visible light. Pulse shaping is used to compensate dispersion to deliver the shortest possible pulses at the foci of microscope objectives for nonlinear biomedical imaging and laser machining, and to achieve distortion-free propagation of femtosecond pulses over $50 \mathrm{~km}$ fibre spans. Specializations of optical pulse shaping geometries have been engineered to realize commercial wavelength-selective switches and gain equalizers that are widely deployed within today's lightwave communications network; some of these specializations were subsequently generalized to provide full pulse shaping capability in robust, fibre optic compatible formats that have now become increasingly popular for applications in microwave photonics. These commercial implementations [224] provide a good calibration for the technical state of the art: simultaneous amplitude and phase shaping is possible across the 5 $\mathrm{THz}$ bandwidth of the lightwave C-band with a spectral resolution of $10 \mathrm{GHz}$, enabling generation of waveforms where the number of independently controllable temporal features (the time-bandwidth product) is of the order of 500 . Further advances in shaping light on an ultrafast time scale will enhance current applications and enable new applications beyond the broad set already established.

\section{Current and future challenges}

Although pulse shaping methods already enable generation of light that is highly structured both in frequency and time, fields that may be realized are still far from arbitrary. For example, most pulse shaping works in the group-of-lines regime, where the ultrafast field is controlled at a resolution

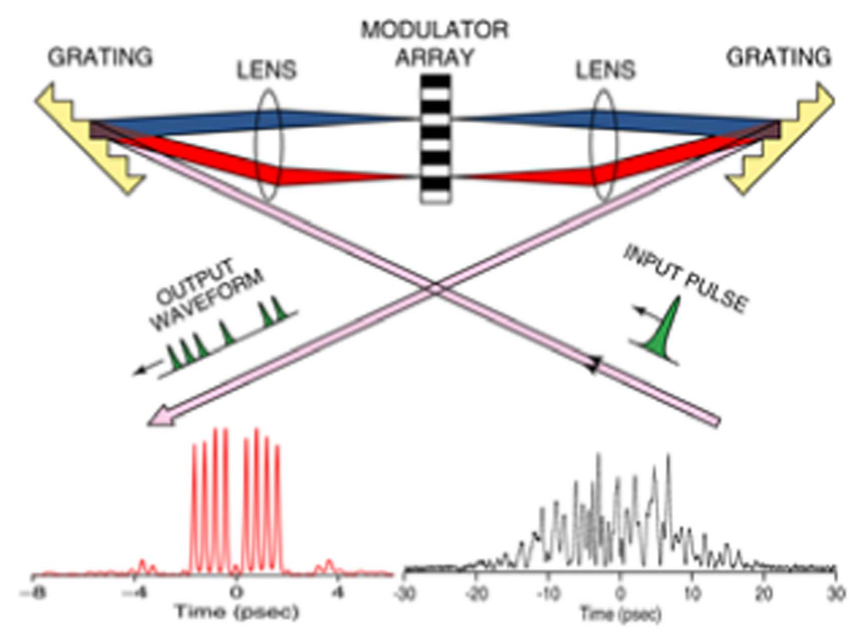

Figure 33. Femtosecond pulse shaper arrangement and representative ultrafast waveforms.

that is coarse compared to the spacing of individual optical frequency components. A fundamental consequence is that shaped waveforms occur in isolated bursts much shorter than the repetition period. One important direction in pulse shaping over the last decade was inspired by the development of optical frequency comb lasers, awarded the 2005 Nobel Prize in Physics for their tremendous impact on precision frequency metrology and allied fields. Optical frequency comb sources highlight the discrete nature of the mode-locked spectrum and provide a stable phase relationship between adjacent pulses in a mode-locked train [225]. This led to the quest for line-byline shaping, also termed optical arbitrary waveform generation, in which spectral control is exercised independently on individual comb lines, with the consequence that the shaped field now expands to fill time [226, 227]. A challenge was that mode-locked laser frequency combs generally have line spacing below $\sim 1 \mathrm{GHz}$, too fine to be resolved by most pulse shapers. Therefore, line-by-line shaping was achieved by using alternate frequency comb sources, such as those based on strong external phase modulation of a single frequency laser. Such electro-optic combs offer frequency spacing into the tens of $\mathrm{GHz}$ range and substantial versatility, but still lack the high stability of mode-locked laser combs.

There are several additional limitations. Shaped pulses are still constrained in both group-of-lines and line-by-line regimes to repeat periodically at the repetition rate of the input laser. The reprogramming time of the pulse shaper is itself generally limited to milliseconds, orders of magnitude slower than the repetition rate of mode-locked lasers and other frequency combs. Therefore, applications in telecommunications and radio-frequency systems that may require new information to be imprinted on successive waveforms are not supported. The number of individual control elements in pulse shapers, although substantial, remains much below the tens or hundreds of thousands of spectral lines found in some frequency comb sources. Existing methods focus predominantly on shaping the phase and amplitude of the field envelope, whereas shaping control at the optical carrier level is needed for ultrafast optics research now pushing beyond the slowly 
varying envelope approximation for manipulation of singlecycle visible light and sub-femtosecond short wavelength fields. Conventional pulse shaping is strictly one-dimensional, meaning that light is structured only in time (equivalently optical frequency); extension to multiple dimensions, allowing programmable generation of nonseparable space-time fields, could enable control of propagation in complex, highly scattering media or of coupling to resonant nanophotonic antennas. Finally, miniaturization of pulse shapers (including the source laser) is highly desirable for certain applications.

\section{Advances in science and technology to meet challenges}

Strong momentum in the field of photonic integration provides opportunities to address several (not all) of the challenges mentioned. Already several groups have demonstrated pulse shaping functionality in chip-level devices. Different material platforms bring different advantages and disadvantages. A pulse shaper with an impressive 64 channels of phase and amplitude control has been reported in silica integrated optics, but with thermo-optic control limited to around the millisecond level [228]. Pulse shapers based on indium phosphide (InP) have also been explored. InP technology offers the possibility of active devices such as on-chip lasers and optical amplifiers as well as modulator structures capable of operation beyond $10 \mathrm{GHz}$ and is deployed for today's coherent lightwave communications, but experiences greater challenges compared to silica in demultiplexing closely spaced wavelength channels. The existence of fast modulators offers the tantalizing possibility of achieving independent updates of successively shaped waveforms, but due to extreme challenges associated with bringing in a large multiplicity of GHz-class electrical control signals, will only be achieved with substantial investment to realize either tight co-packaging or even on-chip electronic-photonic integration of high speed electronic drivers. In any integrated technology, spectral resolution will likely be limited to ten or a few tens of $\mathrm{GHz}$, so high repetition rate sources are desired. Here frequency combs generated via nonlinear wave mixing in optical microresonators are a promising possibility [229]. Such 'micro-combs' have been demonstrated in chip-level structures based on silicon nitride, high index glass, and others, and offer repetition rates from tens to even hundreds of $\mathrm{GHz}$. As one example, our group at Purdue is working with an industrial collaborator towards the realization of a hybrid packaged silicon nitride micro-comb feeding an InP pulse shaper with sub-microsecond reprogramming times for applications in agile RF photonic filtering [230].

For other purposes, free-space pulse shapers are likely to continue to offer performance levels unattainable with integrated approaches. LCOS SLMs are now available with $>10^{6}$ control pixels arranged in a $2 \mathrm{D}$ geometry, orders of (a)

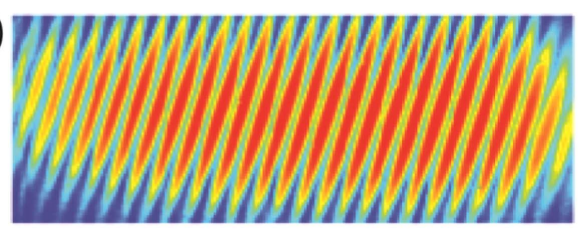

(b)

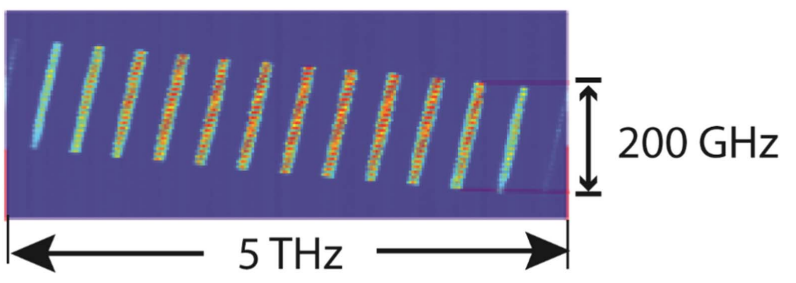

Figure 34. Dispersion of optical frequencies in 2D at the Fourier plane of a pulse shaper, without (a) and with (b) spectral shaping. Adapted with permission from [231]. Copyright 2008 the Optical Society.

magnitude higher compared to previously utilized 1D SLMs or integrated optics. The vastly increased pixel count opens many new possibilities, the most obvious of which is attainment of a larger time-bandwidth product. A pulse shaper with an innovative cross-dispersion arrangement that separates optical frequency components in 2D has achieved a timebandwidth product of 1600, limited by the source (figure 34) [231]. Orders of magnitude higher should be possible. A second possibility is to generate a spatial array of waveforms that are shaped independently in time, i.e. programmable space-time fields to investigate the ability to achieve focusing simultaneously in space and time, deep within highly scattering media such as tissues.

\section{Concluding remarks}

In this article we have very briefly considered structuring ultrafast light fields in time via optical pulse shaping, including research challenges and prospects. Many applications have already become common, both in optical technology and in ultrafast optical science, and more are expected. Additional impact is expected by connecting to other concepts discussed elsewhere in this roadmap, such as exploring spatial degrees of freedom for arbitrary ultrafast space-time fields, bringing in polarization degrees of freedom, and generalizing pulse shaping to nonclassical light. Research on such connections is already underway but is still in its early stages.

\section{Acknowledgments}

This work is supported in part by NSF grants ECCS-1407620 and ECCS-1509578, AFOSR grant FA9550-15-1-0211, and DARPA grant W31P40-13-1-0018. 


\section{Tailoring light at the source}

\section{Andrew Forbes}

University of the Witwatersrand

\section{Status}

Structured light may be created by a variety of techniques external to the source [232], including all the eigenmodes typically found from sources [233], e.g., free-space modes in the form of Laguerre-Gaussian, Bessel-Gaussian and Hermite-Gaussian beams, the linearly polarized modes of optical fibres, waveguide modes and so on. Using SLMs, such eigenmodes can be created with very high fidelity, mimicking complex sources. Historically, refractive and diffractive elements have been used to create novel light fields, for example, the creation of flat-top beams for laser materials processing, employing intensity shaping by the dynamic phase of light [234]. Because such elements modulate only the phase, a two-step approach is often employed. Complex amplitude modulation with liquid crystal devices (e.g., SLMs) has allowed a myriad of novel beams to be created easily with rewritable holograms in a single step, replacing the expensive and labour intensive 'hard-coded' glass optical elements. The geometric phase of light may also be used to tailor light and has become a ubiquitous tool for the creation of vector vortex beams, using so-called q-plates, although of course this design approach is not restricted to a particular class of beam.

The aforementioned tools are generally used for the control of low-intensity beams external to the source. This truism may be extended even to high-dimensional entanglement experiments with spatial modes of light: the quantum state is typically determined by post selection rather than engineered at the source. Such structured light fields have been used in a variety of applications as detailed in this roadmap. However, in many applications there is a need to tailor the light at the source, which in the case of lasers we will refer to as intra-cavity mode control.

Laser cavities are natural filters of noise, and thus hold promise for the generation of ultra-pure modes. They are also able to compensate directly for shaping-induced losses, vital in applications where photons are precious (e.g., remote laser operation). Many decades ago amplitude masking was used to produce Laguerre-Gaussian and Hermite-Gaussian modes from gas and solid state lasers; later phase-only elements were used to create arbitrary beam profiles from such lasers [234], while more modern approaches combine these tools in the form of intra-cavity SLMs [235] for on-demand mode control. Many exciting advances have been made, notably the controlled generation of OAM modes and vector vortex beams directly from solid state laser cavities [236]. Such laser systems have found many applications, for example, in laser machining with structured light.
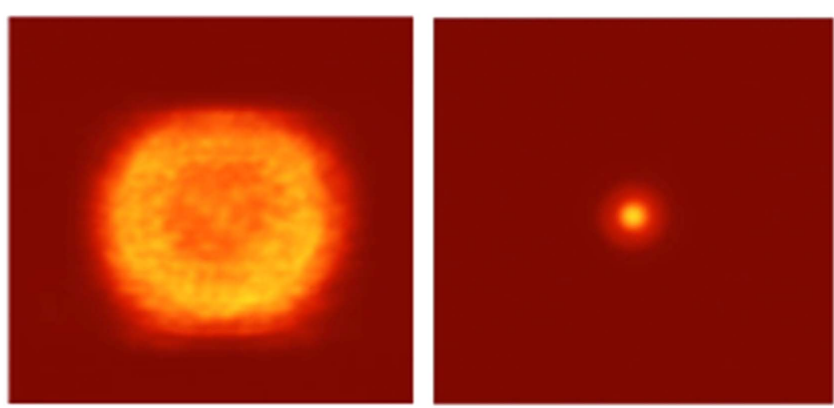

Figure 35. Structured light pattern (left) from a high energy laser producing $5.3 \mathrm{~J}$, compared to the default Gaussian beam from the same laser (right) with $250 \mathrm{~mJ}$. The left-hand pattern was created using an intra-cavity diffractive mirror. Both shown at the same scale.

\section{Current and future challenges}

The challenge is to create robust sources capable of producing spatial modes of a desired type, on demand, and with high purity. The demonstration of a digital laser [235] was a positive step in this direction, but this is a bulky solution that is limited to low power applications.

Importantly, in many applications the demand on the source is high power or compactness, the latter to facilitate integrated photonic solutions. No laboratory demonstrations to date have satisfied all these criteria in one device.

Ironically, it is the mode purity that also remains a challenge. The standard laser cavity design approach guarantees that the desired mode has the lowest loss, but does not speak to the mode competition due to other low-loss modes. As a result, it remains a challenge to ensure that the cavity produces a mode of sufficient purity.

Only recently has the geometric phase control of light been employed as an intra-cavity mode-selecting tool, producing high-order Poincaré sphere beams, and controlling the helicity of OAM modes [236]. The advantage lies in the degrees of freedom available to select a multitude of modes from the same device. Controlling the spatial intensity, phase and polarization remains a topic of intense research today. Polarization control has mostly been limited to cylindrical vector vortex beams by exploiting birefringence in certain laser crystals, but no examples exist of laser cavities that produce arbitrary spatial polarization patterns.

One of the main challenges is to miniaturize such systems to achieve compactness, and to find exotic approaches beyond conventional laser resonators to enable integrated onchip sources of structured light. Finally, most research has concentrated on the generation of the mode, with emphasis on purity and mode selection, but little attention has been paid to the efficiency and power output of the system.

\section{Advances in science and technology to meet challenges}

Integrated and compact solutions for mode control at the source require technologies that enable miniaturization. One exciting prospect is novel structured materials, appropriately engineered to generate structured light [237]. Developments 
in metamaterials [21] and nanostructured materials may hold promise in this regard [109], particularly for integration with fibre optical delivery of structured fields, while early work on organic laser systems suggests a new technology in the future for energy efficient sources of structured light [238]. Integrated silicon photonics, with on-chip generation of novel light fields, have already been demonstrated [111, 239] and applied, for example, in communications.

To achieve compactness in laser systems one requires monolithic design approaches, and here microchip lasers are an exciting technology. While these systems presently do not allow intra-cavity optics, mode control has been demonstrated through gain control (doping) and pump control [240].

A major challenge that remains is to realize these technologies at the high-power levels required for industrial applications: lasers for materials processing can exceed kilowatt power levels. Few of the structured light solutions to date can tolerate such power levels (remember that circulating intensity inside a laser can be many times greater than the output). Technologies such as deformable mirrors, which can handle high powers, do not have the necessary stroke for arbitrary mode creation. This topic remains largely untouched and will surely be a subject of intense research in the future.

\section{Concluding remarks}

Solutions for structured light at the source lag behind externally modulated solutions by some way, but are steadily gaining ground. The merging of materials and light engineering shows tremendous promise for integrated solutions, while monolithic laser designs may address the compactness issues. To date few solutions are viable at the high-power levels and this remains a challenge if structured light beams are to be used in industrial laser applications. 


\section{References}

[1] Nye J F and Berry M V 1974 Dislocations in wave trains Proc. R. Soc. Lond. A336 165-90

[2] Dennis M R, O'Holleran K and Padgett M J 2009 Singular optics: optical vortices and polarization singularities Prog. Opt. 53 293-363

[3] Nye J F 1999 Natural Focusing and Fine Structure Of Light: Caustics and Wave Dislocations (Bristol: Institute of Physics Publishing)

[4] Berry M V 2000 Making waves in physics: three wave singularities from the miraculous 1830s Nature $\mathbf{4 0 3} 21$

[5] Hirschfelder J O, Goebel C G and Bruch L W 1974 Quantized vortices around wavefunction nodes J. Chem. Phys. 61 5456-9

[6] Verbeeck J, Tian H and Schattschneider P 2010 Production and application of electron vortex beams Nature 467 301-4

[7] Soskin M S and Vasnetsov M V 2001 Singular optics Prog. Opt. 42 219-76

[8] Dennis M R, King R P, Jack B, O'Holleran K and Padgett M J 2010 Isolated optical vortex knots Nat. Phys. 6 $118-21$

[9] Foo G, Palacios D M and Swartzlander G A Jr 2005 Optical vortex coronagraph Opt. Lett. 30 3308-10

[10] Klar T A, Jakobs S, Dyba M, Egner A and Hell S W 2000 Fluorescence microscopy with diffraction resolution barrier broken by stimulated emission PNAS 97 8206-10

[11] Arnold A S 20122012 Extending dark optical trapping geometries Opt. Lett. 37 2505-7

[12] Bradshaw D S, Leeder J M, Coles M M and Andrews D L 2015 Signatures of material and optical chirality: origins and measures Chem. Phys. Lett. 626 106-10

[13] Tang Y Q and Cohen A E 2010 Optical chirality and its interaction with matter Phys. Rev. Lett. 104163901

[14] Maurer C, Jesacher A, Fürhapter S, Bernet S and Ritsch-Marte M 2007 Tailoring of arbitrary optical vector beams New J. Phys. 978

[15] Lee M P, Curran A, Gibson G M, Tassieri M, Heckenberg N R and Padgett M J 2012 Optical shield: measuring viscosity of turbid fluids using optical tweezers Opt. Express 20 12127-32

[16] Xie G et al 2015 Performance metrics and design considerations for a free-space optical orbital-angularmomentum-multiplexed communication link Optica 2 357-65

[17] Bliokh K Y and Nori F 2015 Transverse and longitudinal angular momenta of light Phys. Reports 592 1-38

[18] Andersson M, Berglind E and Björk G 2015 Orbital angular momentum modes do not increase the channel capacity in communication links New J. Phys. 17043040

[19] Coles M M, Williams M D, Saadi K, Bradshaw D S and Andrews D L 2013 Chiral nanoemitter array: a launchpad for optical vortices Laser Photonics Rev. 7 1088-92

[20] Karimi E, Schulz S A, De Leon I, Qassim H, Upham J and Boyd R W 2014 Generating optical orbital angular momentum at visible wavelengths using a plasmonic metasurface Light: Sci. Appl. 3 e167

[21] Pu M et al 2015 Catenary optics for achromatic generation of perfect optical angular momentum Science Adv. 1 e1500396

[22] Schmidt A B, Andrews D L, Rohrbach A, Gohn-Kreuz C, Shatokhin V N, Kiselev V G, Hennig J, von Elverfeldt D and Hövener J-B 2016 Do twisted laser beams evoke nuclear hyperpolarization? J. Magnetic Resonance 268 58-67

[23] Penfield P and Haus H A 1967 Electrodynamics of Moving Media (Cambridge, MA: MIT Press)

[24] Robinson F N H 1975 Electromagnetic stress and momentum in matter Phys. Rep. 16 313-54
[25] Einstein A and Laub J 1908 Über die im elektromagnetischen Felde auf ruhende Körper ausgeübten ponderomotorischen Kräfte Ann. Phys. 26 541-50 reprinted in translation: 'On the ponderomotive forces exerted on bodies at rest in the electromagnetic field,' The Collected Papers of Albert Einstein, vol 2 Princeton University Press, Princeton, NJ (1989)

[26] Mansuripur M 2013 The force law of classical electrodynamics: Lorentz versus Einstein and Laub Optical Trapping and Optical Micro-manipulation X ed K Dholakia and G C Spalding Proc. SPIE 8810 88100K $\sim 1: 18$

[27] Kemp B A 2011 Resolution of the Abraham-Minkowski debate: implications for the electromagnetic wave theory of light in matter J. Appl. Phys. 109111101

[28] Mansuripur M 2013 On the foundational equations of the classical theory of electrodynamics Resonance 18 130-55

[29] Balazs N L 1953 The energy-momentum tensor of the electromagnetic field inside matter Phys. Rev. 91 408-11

[30] Loudon R 2004 Radiation pressure and momentum in dielectrics Fortschr. Phys. 52 1134-40

[31] Shockley W and James R P 1967 'Try simplest cases' discovery of 'hidden momentum' forces on 'magnetic currents' Phys. Rev. Lett. 18 876-9

[32] Griffiths D J and Hnizdo V 2013 Mansuripur's paradox Am. J. Phys. 81 570-3

[33] Brevik I 1979 Experiments in phenomenological electrodynamics and the electromagnetic energy-momentum tensor Phys. Rep. 52 133-201

[34] Loudon R 2002 Theory of the radiation pressure on dielectric surfaces J. Mod. Opt. 49 821-38

[35] Baxter C and Loudon R 2010 Radiation pressure and the photon momentum in dielectrics J. Mod. Opt. 57 830-42

[36] Barnett S M and Loudon R 2010 The enigma of optical momentum in a medium Phil. Trans. R. Soc. A 368 927-39

[37] Barnett S M and Loudon R 2015 Theory of radiation pressure on magneto-dielectric materials New J. Phys. 17063027 pp 1-16

[38] Mansuripur M, Zakharian A R and Wright E M 2013 Electromagnetic-force distribution inside matter Phys. Rev. A 88023826 pp 1-13

[39] Allen L, Barnett S M and Padgett M J 2003 Optical Angular Momentum (Bristol: Institute of Physics Publishing)

[40] Allen L, Beijersbergen M W, Spreeuw R J C and Woerdman J P 1992 Orbital angular momentum of light and the transformation of Laguerre-Gaussian laser modes Phys. Rev. A 458185

[41] Simpson N B, Dholakia K, Allen L and Padgett M J 1997 Mechanical equivalence of spin and orbital angular momentum of light: an optical spanner Opt. Lett. 2252

[42] Mansuripur M 2005 Angular momentum of circularly polarized light in dielectric media Opt. Express 13 5315-24

[43] Mansuripur M 2008 Electromagnetic force and torque in ponderable media Opt. Express 16 14821-35

[44] Barnett S M 2010 Rotation of electromagnetic fields and the nature of optical angular momentum J. Mod. Opt. 57 1339-43

[45] Mansuripur M, Zakharian A R and Wright E M 2011 Spin and orbital angular momenta of light reflected from a cone Phys. Rev. A 84033813 pp 1-12

[46] Mansuripur M 2011 Spin and orbital angular momenta of electromagnetic waves in free space Phys. Rev. A 84033838 pp 1-6

[47] Berry M V, Jeffrey M R and Mansuripur M 2005 Orbital and spin angular momentum in conical diffraction J. Opt. A. 7 $685-90$

[48] Jackson J D 1999 Classical Electrodynamics 3rd edn (New York: Wiley) 
[49] Mansuripur M 2015 Energy and linear and angular momenta in simple electromagnetic systems Optical Trapping and Optical Micromanipulation XII ed K Dholakia and G C Spalding Proc. SPIE vol 9548 95480K 1:24

[50] Kristensen M and Woerdman J P 1994 Is photon angular momentum conserved in a dielectric medium? Phys. Rev. Lett. 72 2171-4

[51] Mansuripur M 2014 The charge-magnet paradoxes of classical electrodynamics Spintronics VII ed H J Drouhin, J E Wegrowe and M Razeghi Proc. SPIE vol 9167 91670J $\sim 1: 12$

[52] Padgett M J 2004 The mechanism for energy transfer in the rotational frequency shift of a light beam J. Opt. A. 6 S263-5

[53] Mansuripur M 2012 Angular momentum exchange between light and material media deduced from the Doppler shift Optical Trapping and Optical Micromanipulation IX ed K Dholakia and G C Spalding Proc. SPIE vol 8458845805 $\sim 1: 8$

[54] Gabor D 1948 A new microscopic principle Nature 161 $777-8$

[55] Lohmann A W and Paris D P 1967 Binary fraunhofer holograms, generated by computer Appl. Opt. 6 1739-48

[56] Yeh P and Gu C 2010 Optics of liquid crystal displays (Hoboke, NJ: Wiley)

[57] Davis J and Moreno I 2014 Generation of Laser Beams by Digital Holograms'. Laser Beam Propagation-Generation and Propagation of Customized Light ed A Forbes (Boca Raton, FL: CRC Press) pp 175-204 ch 6

[58] Mair A, Vaziri A, Weihs G and Zeilinger A 2001 Entanglement of the orbital angular momentum states of photons Nature 412 313-6

[59] Galvez E J 2015 Light Beams with Spatially Variable Polarization (Photonics, Fundamentals of Photonics and Physics) ed D L Andrews vol 1 1st edn (Hoboken, NJ: Wiley) pp 61-76

[60] Dholakia K and Čižmár T 2011 Shaping the future of manipulation Nat. Photon. 5 335-42

[61] Padgett M and Bowman R 2011 Tweezers with a twist Nat. Photon. 5 343-8

[62] Woerdemann M, Alpmann C, Esseling M and Denz C 2013 Advanced optical trapping by complex beam shaping Laser \& Photonics Reviews 7 839-54

[63] Richardson D J, Fini J M and Nelson L E 2013 Spacedivision multiplexing in optical fibres' Nat. Photon. 7 354-62

[64] Lazarev G, Hermerschmidt A, Krueger S and Osten S 2013 LCOS spatial light modulators: trends and applications Optical Imaging and Metrology: Advanced Technologies ed W Osten and N Reingand (Hoboken, NJ: Wiley)

[65] Steinlechner F, Hermos N, Pruneri V and Torres J P 2016 Frequency conversion of structured light Sci. Rep. 621390

[66] Stalder M and Schadt M 1996 Linearly polarized light with axial symmetry generated by liquid-crystal polarization converters Opt. Lett. 211948

[67] Marrucci L, Manzo C and Paparo D 2006 Optical spin-toorbital angular momentum conversion in inhomogeneous anisotropic media Phys. Rev. Lett. 96163905

[68] Dorn R, Quabis S and Leuchs G 2003 Sharper focus for a radially polarized light beam Phys. Rev. Lett. 91233901

[69] Bauer T et al 2015 Observation of optical polarization Möbius strips Science 347964

[70] Novotny L, Beversluis M R, Youngworth K S and Brown T G 2001 Longitudinal field modes probed by single molecules Phys. Rev. Lett. 865251

[71] Rotenberg N and Kuipers L 2014 Mapping nanoscale light fields Nature Photon. 8919

[72] Bauer T, Orlov S, Peschel U, Banzer P and Leuchs G 2014 Nanointerferometric amplitude and phase reconstruction of tightly focused vector beams Nature Photon. 823
[73] Wozniak P, Banzer P and Leuchs G 2015 Selective switching of individual multipole resonances in single dielectric nanoparticles Laser Photon. Rev. 9231

[74] Bautista G, Huttunen M J, Mäkitalo J, Kontio J M, Simonen J and Kauranen M 2012 Second-harmonic generation imaging of metal nano-objects with cylindrical vector beams Nano Lett. 123207

[75] Zhan Q 2009 Cylindrical vector beams: from mathematical concepts to applications Adv. Opt. Photon. 1 1-57

[76] D'Ambrosio V, Nagali E, Walborn S P, Aolita L, Slussarenko S, Marrucci L and Sciarrino F 2012 Complete experimental toolbox for alignment-free quantum communication Nat. Commun. 3961

[77] Beckley A M, Brown T G and Alonso M A 2010 Full poincaré beams Opt. Express 18 10777-85

[78] Karimi E et al 2010 Spin-orbit hybrid entanglement of photons and quantum contextuality Phys. Rev. A 82022115

[79] Freund I 2005 Cones, spirals, and Möbius strips, in elliptically polarized light Opt. Commun. 249 7-22

[80] Galvez E J, Khadka S, Schubert W H and Nomoto S 2012 Poincaré-beam patterns produced by nonseparable superpositions of Laguerre-Gauss and polarization modes of light Appl. Opt. 51 2925-34

[81] Cardano F et al 2012 Polarization pattern of vector vortex beams generated by $q$-plates with different topological charges Appl. Opt. 51 C1-6

[82] Geng J 2011 Structured-light 3D surface imaging: a tutorial Adv. Opt. Photon. 3 128-33

[83] Gustafsson M 2000 Surpassing the lateral resolution limit by a factor of two using structured illumination microscopy J. Microscopy 198 82-7

[84] Maurer C, Jesacher A, Bernet S and Ritsch-Marte M 2010 What spatial light modulators can do for optical microscopy Laser Photon. Rev. 5 81-101

[85] Shapiro J H and Boyd R W 2012 The physics of ghost imaging Quantum Inf. Process 11 949-93

[86] Gatti A, Brambilla E, Bache M and Lugiato L A 2004 Correlated imaging, quantum and classical Phys. Rev. A 7010

[87] Shapiro J 2008 Computational ghost imaging Phys. Rev. A 78 061802

[88] Duarte M F, Davenport M A, Takhar D, Laska J N, Ting S, Kelly K F and Baraniuk R G 2008 Single-pixel imaging via compressive sampling IEEE Signal Process. Mag. 25 83-91

[89] Candes E J and Tao T 2015 Near-optimal signal recovery from random projections: universal encoding strategies? IEEE Trans. Inform. Theory 52 5406-25

[90] Morris P A, Aspden R S, Bell J E C, Boyd R W and Padgett M J 2015 Imaging with a small number of photons Nat. Commun. 65913

[91] Lemos G B, Borish V, Cole G D, Ramelow S, Lapkiewicz R and Zeilinger A 2014 Quantum imaging with undetected photons Nature 512 409-12

[92] Edgar M P, Gibson G M, Bowman R W, Sun B, Radwell N, Mitchell K J, Welsh S S and Padgett M J 2015 Simultaneous real-time visible and infrared video with single-pixel detectors Sci. Rep. 5 10669-8

[93] Mertz J 2010 Introduction to optical microscopy. Roberts

[94] Sheppard C J and Shotton D M 1997 Confocal Laser Scanning Microscopy (Milton Park: BIOS Scientific Publishers)

[95] Heintzmann R and Gustafsson M G 2009 Subdiffraction resolution in continuous samples Nat. Photon. 3 362-4

[96] Shoham S 2010 Optogenetics meets optical wavefront shaping Nat. Methods 7 798-9

[97] Huisken J, Swoger J, Del Bene F, Wittbrodt J and Stelzer E H 2004 Optical sectioning deep inside live embryos by selective plane illumination microscopy Science 305 1007-9 
[98] Dodt H U, Leischner U, Schierloh A, Jährling N, Mauch C P and Deininger K ... Becker K 2007 Ultramicroscopy: three-dimensional visualization of neuronal networks in the whole mouse brain Nat. Methods 4 331-6

[99] Vellekoop I M and Mosk A P 2007 Focusing coherent light through opaque strongly scattering media Opt. Lett. 32 2309-11

[100] Hell S W 2015 Nanoscopy with focused light (Nobel Lecture) Angew. Chem. Int. Ed. 54 8054-66 Review

[101] Jesacher A and Ritsch-Marte M Synthetic holographic microscopy Contemp. Phys. in press

[102] Kumar S, Harrison N, Richards-Kortum R and Sokolov K 2007 Plasmonic nanosensors for imaging intracellular biomarkers in live cells Nano Lett. 7 1338-43

[103] Lohmann A W, Dorsch R G, Mendlovic D, Ferreira C and Zalevsky Z 1996 Space-bandwidth product of optical signals and systems JOSA A 13 470-3

[104] Niv A, Biener G, Kleiner V and Hasman E 2005 Spiral phase elements obtained by use of discrete space-variant subwavelength gratings Opt. Commun. 251 306-14

[105] Knöner G, Parkin S, Nieminen T A, Loke V L Y, Heckenberg N R and Rubinsztein-Dunlop H 2007 Integrated optomechanical microelements Opt. Express 15 5521-30

[106] Yu N, Genevet P, Kats M K, Aieta F, Tetienne J P, Capasso F and Gaburro Z 2011 Light Propagation with Phase Discontinuities: Generalized Laws of Reflection and Refraction Science 334333

[107] Zhao Z, Wang J, Li S and Wilner A E 2013 Metamaterialsbased broadband generation of orbital angular momentum carrying vector beams Opt. Lett. 38 932-4

[108] Sun J, Zeng J and Litchinitser N M 2013 Twisting light with hyperbolic metamaterials Opt. Express 21 14975-81

[109] Sun J, Wang Xi, Xu T, Kudyshev Z A, Cartwright A N and Litchinitser N M 2014 Spinning Light on the Nanoscale Nano Lett. 142726

[110] Cai W and Shalaev V M 2010 Optical Metamaterials: Fundamentals and Applications (Berlin: Springer)

[111] Cai X et al 2012 Integrated Compact Optical Vortex Beam Emitters Science 338 363-6

[112] Miao P, Zhang Z, Sun J, Walasik W, Longhi S, Litchinitser N M and Feng L 2016 Orbital angular momentum microlaser Science 353 464-7

[113] Chong K E et al 2015 Polarization-Independent Silicon Metadevices for Efficient Optical Wavefront Control Nano Lett. 15 5369-5374

[114] Shalaev M I, Sun J, Tsukernik A, Pandey A, Nikolskiy K and Litchinitser N M 2015 High-Efficiency All-Dielectric Metasurfaces for Ultracompact Beam Manipulation in Transmission Mode Nano Lett. 15 6261-6

[115] Radwell N, Walker G and Franke-Arnold S 2013 Phys. Rev. A 88043409

Turpin A, Polo J, Loiko Y V, Küber J, Schmaltz F, Kalandjiev T K, Ahufinger V, Birlkl G and Mompart J 2015 Opt. Express 231638

Ozeri R, Khaykovich L and Davidson N 1999 Phys. Rev. A 59 R 1750

[116] Ramanathan A, Wright K C, Muniz S R, Zelan M, Hill W T, Lobb C J, Helmerson K, Phillips W D and Campbell G K 2011 Superflow in a Toroidal Bose-Einstein Condensate: An Atom Circuit with a Tunable Weak Link Phys. Rev. Lett. 106130401

[117] Cronin A D, Schmiedmayer J and Pritchard D E 2009 Optics and interferometry with atoms and molecules Rev. Mod. Phys. 801051

Meystre P 2001 Atom Optics (Berlin: Spinger) and references therein
[118] Soskin M S and Vasnetsov M V 2001 Singular optics Prog. Opt. 42

[119] Andersen M F, Ryu C, Clade P, Natarajan V, Vaziri A, Helmerson K and Phillips W D 2006 Phys. Rev. Lett. 97 170406

Beattie et al 2013 PRL 110025301

[120] Wright K C, Leslie L S and Bigelow N P 2008 Phys. Rev. A 77041601

Wright K C, Leslie L S and Bigelow N P 2008 Phys. Rev. A 78053412

Wright K C et al 2009 Phys. Rev. Lett. 102030405

Leslie L S et al 2009 Phy. Rev. Lett. 103250401

Hansen A, Schultz J T and Bigelow N P 2013 Coherence and Quantum Optics (Washington, DC: Optical Society of America)

Hansen A et al 2012 Frontiers in Optics 2012/Laser Science XXVIII, OSA Technical Digest (online) (Washington, DC: Optical Society of America) paper LTu1I.2

[121] Dalibard J, Gerbier F, Juzeliūnas G and Öhberg P 2001 Colloquium: Artificial gauge potentials for neutral atoms Rev. Mod. Phys. 831523

[122] Milione G, Sztul H I, Nolan D A and Alfano R R 2011 Higher-Order Poincaré Sphere, Stokes Parameters, and the Angular Momentum of Light Phys. Rev. Lett. 107 053601

[123] Galvez E J et al 2014 Generation of isolated asymmetric umbilics in light's polarization Phys. Rev. A 89 and references therein031801R

[124] Schultz J T, Hansen A and Bigelow N P 2014 A Raman waveplate for spinor Bose-Einstein condensates Opt. Lett. 394271

[125] Nicolas A, Veissier L, Giner L, Giacobino E, Maxein D and Laurat J 2014 A quantum memory for orbital angular momentum photonic qubits Nat. Photon. 8 234-8

[126] Bakr W S, Gillen J I, Peng A, Foelling S and Greiner M 2009 A quantum gas microscope for detecting single atoms in a Hubbard-regime optical lattice Nature 462 74-7

[127] Torres J P and Torner L 2007 Twisted Photons: Applications of Light with Orbital Angular Momentum 1 (New York: Wiley)

[128] Rosales-Guzmán C, Mazilu M, Baumgartl J, Rodríguez-Fajardo V, Ramos-García R and Dholakia K 2013 Collision of propagating vortices embedded within Airy beams J. Opt. 154

[129] Hermosa N, Rosales-Guzmán C and Torres J P 2013 Helicoconical optical beams self-heal Opt. Lett. 38383

[130] Belmonte A and Torres J P 2011 Optical Doppler shift with structured light Opt. Lett. 364437

[131] Rosales-Guzmán C, Hermosa N, Belmonte A and Torres J P 2013 Experimental detection of transverse particle movement with structured light Sci. Rep. 302815

[132] Belmonte A, Rosales-Guzmán C and Torres J P 2015 Measurement of flow vorticity with helical beams of light Optica 21002

[133] Rosales-Guzmán C, Volke-Sepulveda K and Torres J P 2012 Light with enhanced optical chirality Opt. Lett. 373486

[134] Hermosa N, Rosales-Guzmán C, Pereira S F and Torres J P 2014 Nanostep height measurement via spatial mode projection Opt. Lett. 2299

[135] Rosales-Guzmán C, Hermosa N, Belmonte A and Torres J P 2014 Direction-sensitive transverse velocity measurement by phase-modulated structured light beams Opt. Lett. 395415

[136] Rosales-Guzmán C, Hermosa N, Belmonte A and Torres J P 2014 Measuring the translational and rotational velocities of particles in helical motion using structured light $O p t$. Express 2216504 
[137] Seaman B T, Krämer M, Anderson D Z and Holland M J 2007 Atomtronics: Ultracold-atom analogs of electronic devices Phys. Rev. A 75 and references therein023615

[138] Eckel S, Lee J G, Jendrzejewski F, Lobb C J, Campbell G K and Hill W T III 2016 Phys. Rev. A 93 063619

Lee J G, McIlvain B J, Lobb C J and Hill W T III 2013 Sci. Rep. 3

[139] Stickney J A, Anderson D Z and Zozulya A A 2007 Phys. Rev. A 75013608

Labouvie R, Santra B, Heun S, Wimberger S and Ott H 2015 Phys. Rev. Lett. 115050601

[140] Ryu C and Boshier M G 2015 Integrated coherent matter wave circuits New J. Phys. 17092002

[141] Bell T A, Glidden J A, Humbert L, Bromley M W, Haine S A, Davis M J, Neely T W, Baker M A and Rubinsztein-Dunlop H 2016 Integrated coherent matter wave circuits New J. Phys. 18035003

[142] Eckel S, Lee J G, Jendrzejewski F, Murray N, Clark C W, Lobb C J and Campbell G K 2014 Hysteresis in a quantized superfluid 'atomtronic' circuit Nature 506 200-3

[143] Pasienski M and DeMarco B 2008 Opt. Express 16 2176-90 Gaunt A and Hadzibabic Z 2011 Sci. Rep. 2721

[144] Hansen A, Schultz J T and Bigelow N P 2016 Singular atom optics with spinor Bose-Einstein condensates Optica 3 355-61

[145] Kumar A, Anderson N, Phillips W D, Eckel S, Campbell G K and Stringari S 2016 New J. Phys. 18025001 Direct imaging of a digital-micromirror device for configurable microscopic optical potentials. G Gauthier, I Lenton, N McKay Parry, M Baker, M J Davis, H Rubinsztein-Dunlop, and T W Neely. Submitted to Optica

[146] Zozulya A A and Anderson D Z 2013 Principles of an atomtronic battery Phys. Rev. A 88043641

[147] Juan M L, Gordon R, Pang Y, Eftekhari F and Quidant R 2009 Self-induced back-action optical trapping of dielectric nanoparticles Nat. Phys. 5 915-9

[148] Pang Y and Gordon R 2011 Optical trapping of a single protein Nano Lett. 12 402-6

[149] Berthelot J, Aćimović S S, Juan M L, Kreuzer M P, Renger J and Quidant R 2014 Three-dimensional manipulation with scanning near-field optical nanotweezers Nat. Nanotechnol. 9 295-9

[150] Wheaton S, Gelfand R M and Gordon R 2014 Probing the Raman-active acoustic vibrations of nanoparticles with extraordinary spectral resolution Nat. Photonics 9 68-72

[151] García-Vidal F J, Martín-Moreno L, Moreno E, Kumar L K S and Gordon R 2006 Transmission of light through a single rectangular hole in a real metal Phys. Rev. B 74153411

[152] Chen X, Ciracì C, Smith D R and Oh S H 2015 Nanogapenhanced infrared spectroscopy with template-stripped wafer-scale arrays of buried plasmonic cavities Nano Lett. 15 107-13

[153] Berini P 2008 Bulk and surface sensitivities of surface plasmon waveguides New J. Phys. 10105010

[154] Melentiev P N, Afanasiev A E, Kuzin A A, Baturin A S and Balykin V I 2013 Giant optical nonlinearity of a single plasmonic nanostructure Optics Express 21 13896-905

[155] Regmi R, Al Balushi A A, Rigneault H, Gordon R and Wenger J 2015 Nanoscale volume confinement and fluorescence enhancement with double nanohole aperture Sci. Rep. 515852

[156] Lin D and Huang J S 2014 Slant-gap plasmonic nanoantennas for optical chirality engineering and circular dichroism enhancement Opt. Express 22 7434-45

[157] Ashkin A, Dziedzic J M, Bjorkholm J E and Chu S 1986 Observation of a single-beam gradient force optical trap for dielectric particles Opt. Lett. 11 288-90
[158] He H, Heckenberg N R and Rubinsztein-Dunlop H 1995 Optical particle trapping with higher-order doughnut beams produced using high efficiency computer generated holograms J. Mod. Opt. 42 217-23

[159] Grier D G 2003 A revolution in optical manipulation Nature 424 810-6

[160] Thalhammer G, Steiger R, Bernet S and Ritsch-Marte M 2011 Optical macro-tweezers: trapping of highly motile microorganisms J. Opt. 13044024

[161] Knöner G, Parkin S, Nieminen T A, Loke V L Y, Heckenberg N R and Rubinsztein-Dunlop H 2007 Integrated optomechanical microele-ments Opt. Express 15 5521-30

[162] Cizmar T, Mazilu M and Dholakia K 2010 In situ wavefront correction and its application to micromanipulation Nat. Photon. 4 388-94 06

[163] Liberale C, Minzioni P, Bragheri F, Angelis F D, Fabrizio E D and Cristiani I 2007 Miniaturized all-fibre probe for three-dimensional optical trapping and manipulation Nat. Photon. 1 723-7

[164] Bianchi S and Di Leonardo R 2012 A multi-mode fiber probe for holographic micromanipulation and microscopy $L a b$ Chip 12 635-9

[165] Taylor M A, Waleed M, Stilgoe A B, Rubinsztein-Dunlop H and Bowen W P 2015 Enhanced optical trapping via structured scattering Nat. Photon. 9 669-73

[166] Stilgoe A B, Nieminen T A and Rubinsztein-Dunlop H 2015 Energy, momentum and propagation of non-paraxial highorder Gaussian beams in the presence of an aperture $J$. Opt. 17125601

[167] Wang X L et al 2015 Quantum teleportation of multiple degrees of freedom of a single photon Nature 518 516-9

[168] Davis J A, Cottrell D M, Campos J, Yzuel M J and Moreno I 1999 Encoding amplitude information onto phase-only filters Appl. Opt. 38 5004-13

[169] Ramachandran S, Fini J M, Mermelstein M, Nicholson J W, Ghalmi S and Ya M F 2008 Ultra-large effective-area, higher-order mode fibers: a new strategy for high-power lasers Laser Photon. Rev. 2 429-48

[170] Knill E, Laflamme E and Milburn G J 2001 A scheme for efficient quantum computation with linear optics Nature 409 46-52

[171] Brennen G K, Bullock S S and O'Leary D P 2006 J. Quantum Info. Computation 6 436-54

[172] Nielsen M A and Chuang I 2000 Quantum Computation and Quantum Information (New York: Cambridge University Press)

[173] Menicucci N C, van Loock P, Gu M, Weedbrook C, Ralph T C and Nielsen M 2006 Universal Quantum Computation with Continuous-Variable Cluster States Phys. Rev. Lett. 97110501

[174] Bunning T J, Natarajan L V, Tondiglia V P and Sutherland R L 2000 Holographic Polymer-Dispersed Liquid Crystals (H-PDLCs) Ann. Rev. Mat. Sci. 30 83-115

[175] Langford N K et al 2004 Measuring Entangled Qutrits and Their Use for Quantum Bit Commitment Phys. Rev. Lett. 93 053601

[176] Campbell E, Hussain A and Browne D 2012 Phys. Rev. X 2 041021

Brown K, Munro W J and Kendon V 2010 Entropy 12 2268-307

[177] Javadi A et al 2015 Nat. Commun. 68655

[178] De Santis L et al 2016 A solid-state single-photon filter (arXiv:1607.05977)

[179] Krenn M, Huber M, Fickler R, Lapkiewicz R, Ramelow S and Zeilinger A 2014 Generation and confirmation of a $(100 \times 100)$-dimensional entangled quantum system Proc. Natl Acad. Sci. 111 6243-7 
[180] Krenn M, Handsteiner J, Fink M, Fickler R and Zeilinger A 2015 Twisted photon entanglement through turbulent air across Vienna Proc. Natl Acad. Sci. 112 14197-201

[181] Mirhosseini M et al 2015 High-dimensional quantum cryptography with twisted light New J. Phys. 17033033

[182] Berkhout G C G, Lavery M P J, Courtial J, Beijersbergen M W and Padgett M W 2010 Efficient sorting of orbital angular momentum states of light Phys. Rev. Lett. 105153601

[183] Bozinovic N, Yue Y, Ren Y, Tur M, Kristensen P, Huang H, Willner A E and Ramachandran S 2013 Terabit-scale orbital angular momentum mode division multiplexing in fibers Science 340 1545-8

[184] Zhou Z-Q, Hua Y, Liu X, Chen G, Xu J, Han Y, Li C and Guo G 2015 Quantum storage of three-dimensional orbital angular momentum entanglement in a crystal Phys. Rev. Lett. 115070502

[185] Schmiegelow C T, Schulz J, Kaufmann H, Ruster T, Poschinger U G and Schmidt-Kaler F 2016 Transfer of optical orbital angular momentum to a bound electron 7 12998

[186] Malik M, Erhard M, Huber M, Krenn M, Fickler R and Zeilinger A 2016 Multi-photon entanglement in high dimensions Nat. Photon. 10 248-52

[187] Vallone G, D'Ambrosio V, Sponselli A, Slussarenko S, Marrucci L, Sciarrino F and Villoresi P 2014 Free-space quantum key distribution by rotation-invariant twisted photons Phys. Rev. Lett. 113060503

[188] Gibson G, Courtial J, Padgett M, Vasnetsov M, Pas'ko V, Barnett S and Franke-Arnold S 2004 Free-space information transfer using light beams carrying orbital angular momentum Opt. Express 12 5448-56

[189] Wang J et al 2012 Terabit free-space data transmission employing orbital angular momentum multiplexing $\mathrm{Nat}$. Photon. 6 488-96

[190] Willner A E et al 2015 Optical communications using orbital angular momentum beams Adv. Opt. Photon. 7 66-106

[191] Bozinovic N, Yue Y, Ren Y, Tur M, Kristensen P, Huang H, Willner A E and Ramachandran S 2013 Terabit-scale orbital angular momentum mode division multiplexing in fibers Science 340 1545-8

[192] Krenn M, Handsteiner J, Fink M, Fickler R, Ursin R, Malik M and Zeilinger A 2016 Twisted light transmission over 143 kilometers (arXiv:1606.01811)

[193] Malik M, O'Sullivan M, Rodenburg B, Mirhosseini M, Leach J, Lavery M P J, Padgett M J and Boyd R W 2012 Influence of atmospheric turbulence on optical communications using orbital angular momentum for encoding Opt. Express 20 13195-200

[194] Ahmed N et al 2016 Mode-division-multiplexing of multiple Bessel-Gaussian beams carrying orbital-angular-momentum for obstruction-tolerant free-space optical and millimetrewave communication links Sci. Rep. 6

[195] Cai X, Wang J, Strain M J, Johnson-Morris B, Zhu J, Sorel M, O'Brien J L, Thompson M G and Yu S 2012 Integrated compact optical vortex beam emitters Science 338 363-6

[196] McMorran B J, Agrawal A, Anderson I M, Herzing A A Lezec H J, McClelland J J and Unguris J 2011 Electron vortex beams with high quanta of orbital angular momentum Science 331 192-5

[197] Uchida M and Tonomura A 2010 Generation of electron beams carrying orbital angular momentum Nature 464737

[198] Grillo V, Karimi E, Gazzadi G C, Frabboni S, Dennis M R and Boyd R W 2014 Generation of nondiffracting electron bessel beams Phys. Rev. X 4011013

[199] Voloch-Bloch N, Lereah Y, Lilach Y, Gover A and Arie A 2013 Generation of electron airy beams Nature 494 $331-5$
[200] Verbeeck J, Schattschneider P, Lazar S, Stöger-Pollach M, Löffler S, Steiger-Thirsfeld A and Tendeloo G V 2011 Atomic scale electron vortices for nanoresearch Appl. Phys. Lett. 99203109

[201] Ercius P, Harvey T, Pierce J, Chess J, Linck M and McMorran B 2014 Atomic-resolution imaging using Cscorrected vortex beams Microsc. Microanal. 20 84-5

[202] Gallatin G M and McMorran B 2012 Propagation of vortex electron wave functions in a magnetic field Phys. Rev. A 86 012701

[203] Bliokh K Y, Schattschneider P, Verbeeck J and Nori F 2012 Electron vortex beams in a magnetic field: a new twist on landau levels and Aharonov-Bohm states (arXiv:1204.2780)

[204] Asenjo-Garcia A and García de Abajo F J 2014 Dichroism in the interaction between vortex electron beams, plasmons, and molecules Phys. Rev. Lett. 113066102

[205] Idrobo J C and Pennycook S J 2011 Vortex beams for atomic resolution dichroism J. Electron Microsc. (Tokyo) 60 295-300

[206] Xin H L and Zheng H 2012 On-column 2p bound state with topological charge \pm 1 excited by an atomic-size vortex beam in an aberration-corrected scanning transmission electron microscope Microsc. Microanal. 18 711-9

[207] Schattschneider P, Schaffer B, Ennen I and Verbeeck J 2012 Mapping spin-polarized transitions with atomic resolution Phys. Rev. B 85134422

[208] Lloyd S, Babiker M and Yuan J 2012 Quantized orbital angular momentum transfer and magnetic dichroism in the interaction of electron vortices with matter Phys. Rev. Lett. 108074802

[209] Rusz J, Bhowmick S, Eriksson M and Karlsson N 2014 Scattering of electron vortex beams on a magnetic crystal: towards atomic-resolution magnetic measurements Phys. Rev. B 89134428

[210] Ophus C, Ciston J, Pierce J, Harvey T R, Chess J, McMorran B J, Czarnik C, Rose H H and Ercius P A 2016 Efficient linear phase contrast in scanning transmission electron microscopy with matched illumination and detector interferometry Nat. Commun. in press (doi:10.1038/ ncomms10719)

[211] Schattschneider P, Stöger-Pollach M and Verbeeck J 2012 Novel vortex generator and mode converter for electron beams Phys. Rev. Lett. 109084801

[212] Dirac P A M 1948 The theory of magnetic poles Phys. Rev. 74817

[213] Blackburn A M and Loudon J C 2014 Vortex beam production and contrast enhancement from a magnetic spiral phase plate Ultramicroscopy 136 127-43

[214] Béché A, Van Boxem R, Van Tendeloo G and Verbeeck J 2014 Magnetic monopole field exposed by electrons Nat. Phys. 10 26-9

[215] McMorran B J 2009 Electron diffraction and interferometry using nanostructures $P h D$ Dissertation University of Arizona, Tucson, AZ: University of Arizona

[216] Harvey T R, Pierce J S, Agrawal A K, Ercius P, Linck M and McMorran B J 2014 Efficient diffractive phase optics for electrons New J. Phys. 16093039

[217] Grillo V, Gazzadi G C, Karimi E, Mafakheri E, Boyd R W and Frabboni S 2014 Highly efficient electron vortex beams generated by nanofabricated phase holograms Appl. Phys. Lett. 104043109

[218] Shiloh R, Lereah Y, Lilach Y and Arie A 2014 Sculpturing the electron wave function using nanoscale phase masks Ultramicroscopy 144 26-31

[219] Carroll A M 2015 Pattern generators for reflective electronbeam lithography (REBL) Adv. Imaging Electron Phys. ed P W Hawkes vol 188 (Amsterdam: Elsevier) pp 1-23

[220] Petric P, Bevis C, McCord M, Carroll A, Brodie A, Ummethala U, Grella L, Cheung A and Freed R 2010 Reflective electron beam lithography: a maskless ebeam 
direct write lithography approach using the reflective electron beam lithography concept J. Vac. Sci. Technol. B 28 C6C6-6C13

[221] Berry M V and McDonald K T 2008 Exact and geometrical optics energy trajectories in twisted beams $J$. Opt. Pure Appl. Opt. 10035005

[222] Weiner A M 2000 Femtosecond pulse shaping using spatial light modulators Rev. Sci. Instr. 71 1929-60

[223] Weiner A M 2011 Ultrafast optical pulse shaping: a tutorial review Opt. Commun. 284 3669-92

[224] https://finisar.com/optical-instrumentation

[225] Ye J and Cundiff S T 2005 Femtosecond Optical Frequency Comb Technology (New York: Springer)

[226] Jiang Z, Huang C B, Leaird D E and Weiner A M 2007 Optical arbitrary waveform processing of more than 100 spectral comb lines Nat. Photon. 1 463-7

[227] Cundiff S T and Weiner A M 2010 Optical arbitrary waveform generation Nat. Photon. 4 760-6

[228] Fontaine N K et al 2008 Compact $10 \mathrm{GHz}$ loopback arrayedwaveguide grating for high-fidelity optical arbitrary waveform generation Opt. Lett. 33 1714-6

[229] Kippenberg T J, Holzwarth R and Diddams S A 2011 Microresonator-based optical frequency combs Science 332 $555-9$

[230] Metcalf A J, Leaird D E, Jaramillo J, Lal V, Hosseini A, Kish F and Weiner A M 201532 channel, 25 GHz InP integrated pulse shaper with SOA amplitude control IEEE Photonics Conf. (IPC) pp 500-1
[231] Supradeepa V R, Huang C B, Leaird D E and Weiner A M 2008 Femtosecond pulse shaping in two dimensions: towards higher complexity optical waveforms Opt. Express 16 11878-87

[232] Forbes A, Dudley A and McLaren M G 2016 Creation and detection of optical modes with spatial light modulators $A d v$. Opt. Photon. 8 200-27

[233] Forbes A 2014 Laser Beam Propagation: Generation and Propagation of Customised Light (New York: CRC Press)

[234] Belanger P A, Lachance R L and Pare C 1992 Super-Gaussian output from a $\mathrm{CO}_{2}$ laser by using a graded-phase mirror resonator Opt. Lett. 17 739-41

[235] Ngcobo S et al 2013 A digital laser for on-demand laser modes Nat. Commun. 42289

[236] Naidoo D et al 2016 Controlled generation of higherorder Poincaré sphere beams from a laser Nat. Photon. 10327

[237] Litchinitser N 2012 Structured Light Meets Structured Matter Science 337 1054-5

[238] Qian H, Markman B D and Giebink N C 2013 Vector vortex beam emission from organic semiconductor microlasers Appl. Phys. Lett. 103161110

[239] Strain M J et al 2014 Fast electrical switching of orbital angular momentum modes using ultra-compact integrated vortex emitters Nat. Commun. 54856

[240] Naidoo D et al 2015 Radially polarized cylindrical vector beams from a monolithic microchip laser Opt. Eng. 54 111304 